\title{
Adapting Evidence-Based Treatments for Youth in a Community Mental Health Setting: Single Case Design
}

Alexis Quinoy

Virginia Commonwealth University

Follow this and additional works at: https://scholarscompass.vcu.edu/etd

Part of the Clinical Psychology Commons

() The Author

\section{Downloaded from}

https://scholarscompass.vcu.edu/etd/2394

This Thesis is brought to you for free and open access by the Graduate School at VCU Scholars Compass. It has been accepted for inclusion in Theses and Dissertations by an authorized administrator of VCU Scholars Compass.

For more information, please contact libcompass@vcu.edu. 


\title{
ADAPTING EVIDENCE-BASED TREATMENTS FOR YOUTH IN A COMMUNITY MENTAL HEALTH SETTING: SINGLE CASE DESIGN
}

A thesis submitted in partial fulfillment of the requirements for the degree of Master of Science in Psychology at Virginia Commonwealth University.

\author{
By: Alexis M. Quinoy \\ Bachelor of Science \\ The University of Florida
}

\begin{abstract}
Director: Michael A. Southam-Gerow, Ph.D.
Associate Professor

Department of Psychology and Pediatrics
\end{abstract}

Virginia Commonwealth University

Richmond, Virginia

May 2011 


\section{Acknowledgements}

I would like to express my gratitude and appreciation to my advisor and committee chair, Dr. Michael Southam-Gerow. His guidance and unwavering support have been a blessing to me throughout this process. He believed in my abilities and motivated me through difficult personal events by encouraging me to keep "pushing through the marathon." I would also like to thank the other members of my committee, Drs. Bryce McLeod and Melissa Abell for their support and invaluable feedback throughout this process.

I am also grateful to Kelly Pugh, Elizabeth Archer, Priscilla Powell, Shannon Hourigan, Ruth Brown, Emily Wheat Butt, Cassidy Arnold, and Lily Christon Arnold for their unwavering support and much needed humor through my graduate school career. I would also like to thank all of my friends for keeping me grounded and supporting me wherever my life decisions take me.

I also appreciate our participants and families for their involvement in this project. I especially would like to thank everyone at the Chesterfield Community Services Board involved with the project. Without their support, encouragement, and ideas this project would not have been possible.

I would not be where I am today if it wasn't for the unconditional love and support from my wonderful family. I am extremely grateful to my parents, George and Sylvia, whose unwavering love, support, and strength has carried me through life. They have taught me about faith, loyalty, perseverance, dignity, and life balance. I would also like to thank my brother Mike and his wife Allie for all of their love, support, and encouragement. Finally, I express my gratitude for my partner John for his patience, tolerance, humor, love, and support. Our relationship inspires me to challenge myself and grow every day. 
Table of Contents

Page

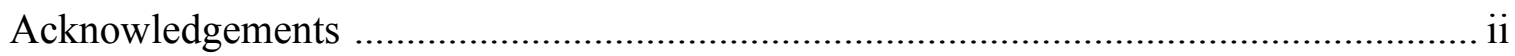

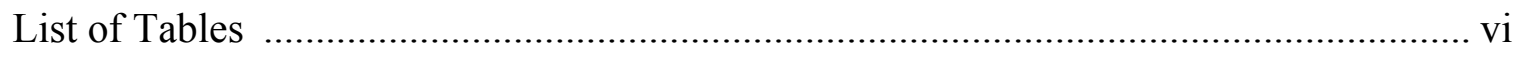



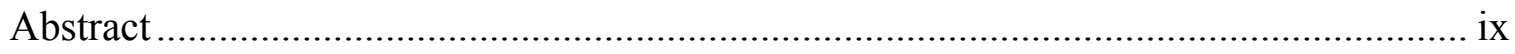

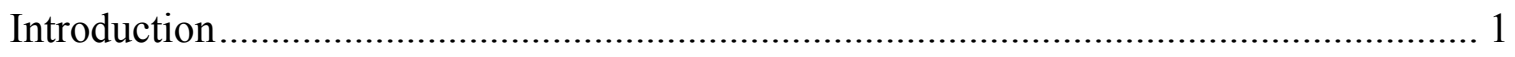

Evidence-Based Treatment Review ............................................................... 2

Transporting EBTs into Community Settings................................................. 7

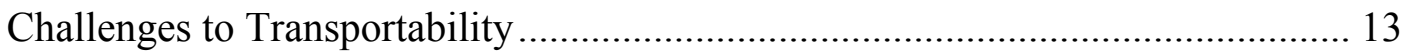

Client level differences .................................................................... 13

Therapist level differences ............................................................. 16

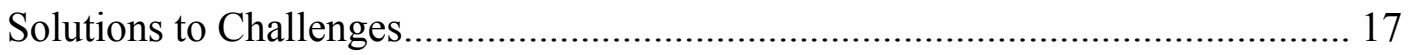

A Partnership Approach to Treatment Adaptation .................................. 18

University-Community Partnership Approach ................................................ 19

Justification of Single Case Design ..................................................... 20

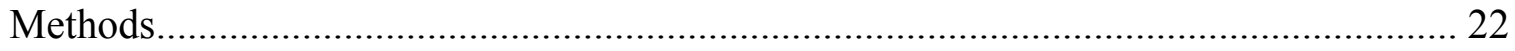



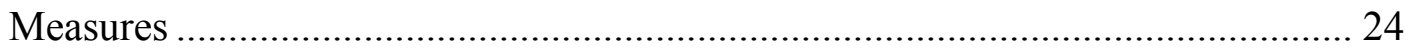

Symptom \& Diagnosis Domain ........................................................ 25

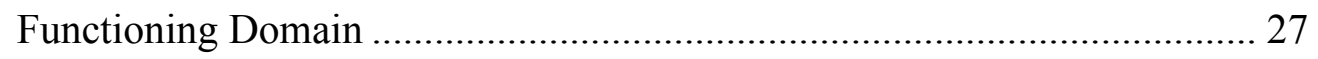

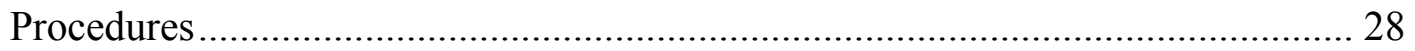

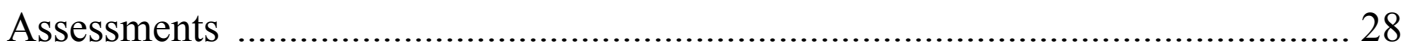




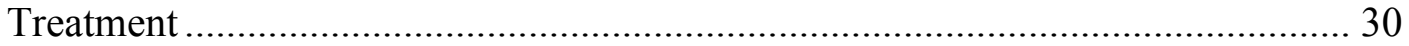



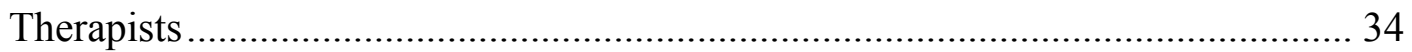

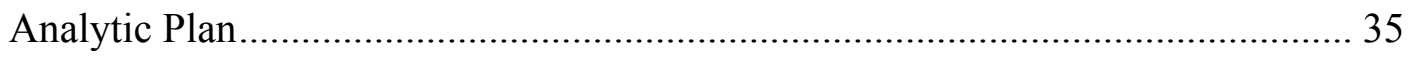

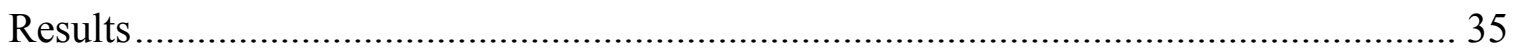

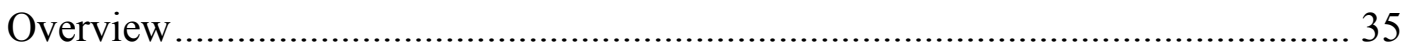



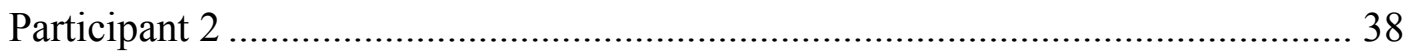

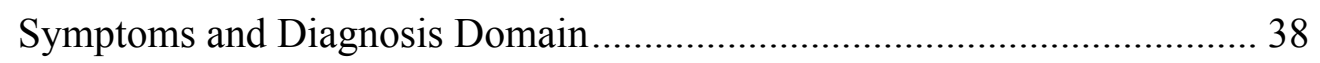

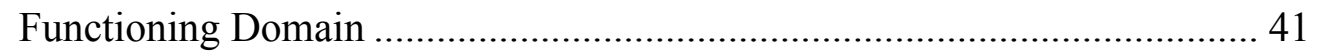

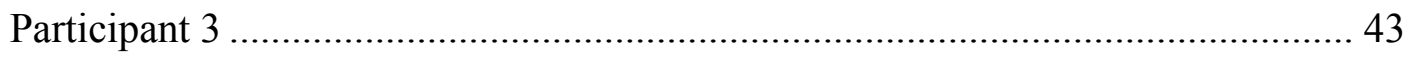

Symptoms and Diagnosis Domain ............................................................. 43

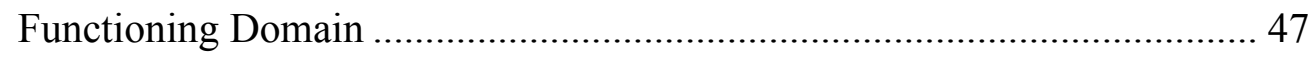

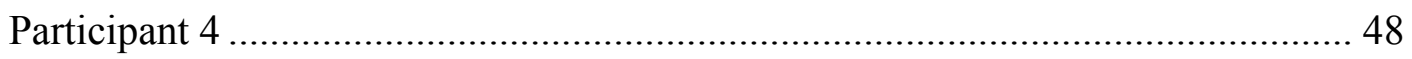

Symptoms and Diagnosis Domain ............................................................ 48

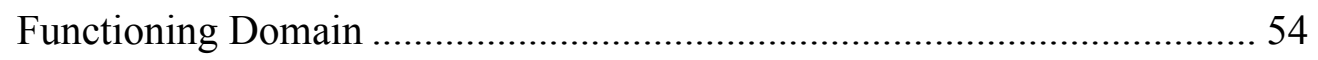

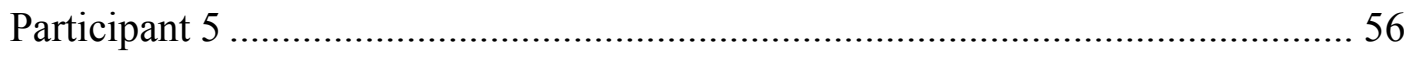

Symptoms and Diagnosis Domain ............................................................... 56

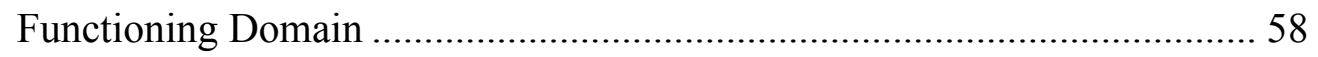

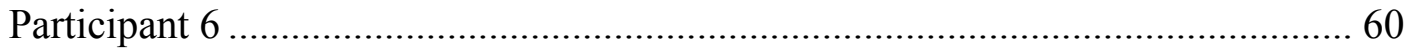

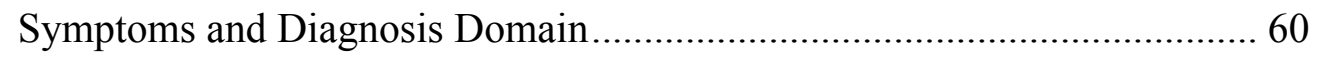

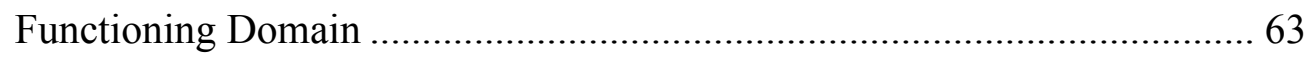

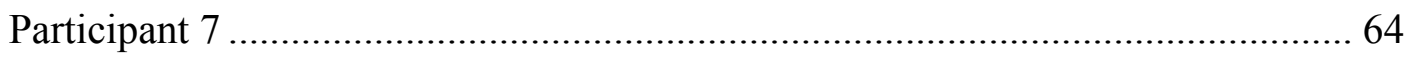




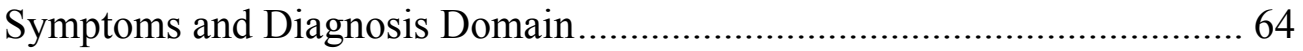

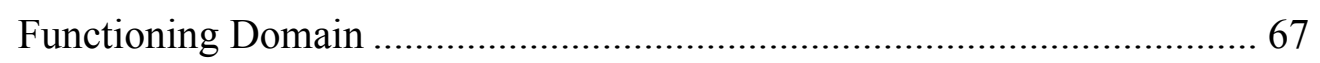

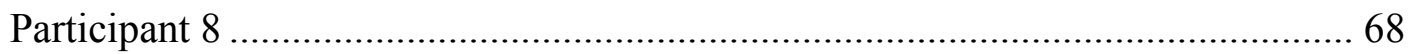

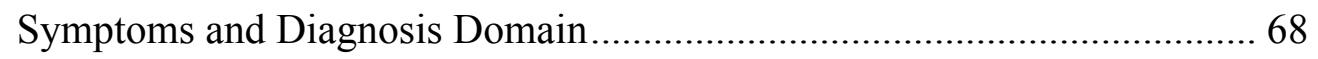

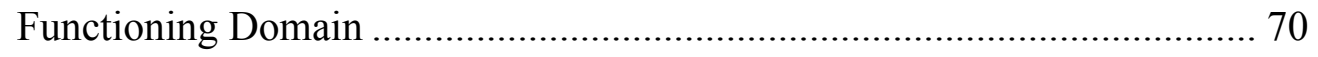

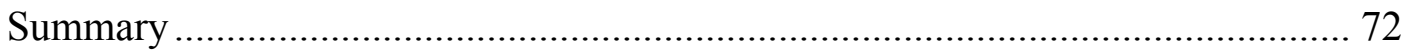

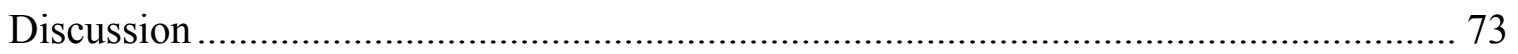

Interpretation and Summary of Results ................................................................ 74

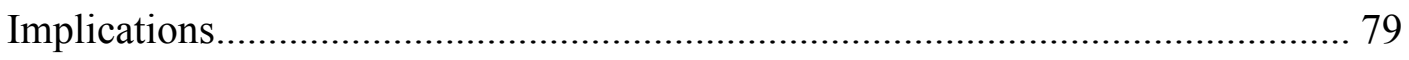

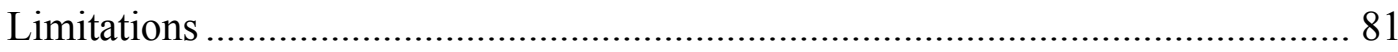

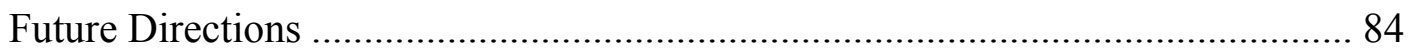

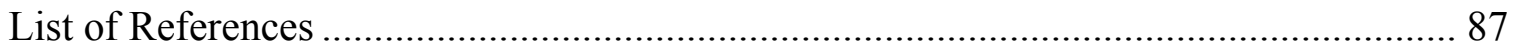

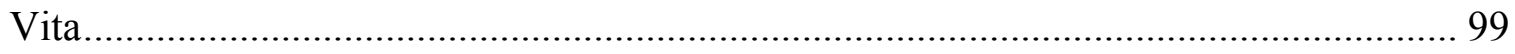




\section{List of Tables}

Page

Table 1. Participant Demographics................................................................................ 23

Table 2. Measurement Battery Composition at Each Assessment Point ........................... 28

Table 3. Treatment Outcome Measures for Each Participant ......................................... 36

Table 4. Summary of Participant Outcomes and Treatment Effectiveness for Participant 2






\section{List of Figures}

Page

Figure 1. RCADS T-scores across baseline, treatment, and follow-up for participant 2 ...... 38

Figure 2. CBCL Internalizing T-scores across baseline, treatment, and follow-up for participant 2

Figure 3. CBCL Anxiety T-scores across baseline, treatment, and follow-up for participant 2

Figure 4. CIS total scale scores across baseline, treatment, and follow-up for participant 2

Figure 5. RCADS T-scores across baseline, treatment, and follow-up for participant $3 \ldots . . .44$

Figure 6. CBCL Total Internalizing T-scores across baseline, treatment, and follow-up for participant 3

Figure 7. CBCL Externalizing T-scores across baseline, treatment, and follow-up for participant 3

Figure 8. CIS total scale scores across baseline, treatment, and follow-up for participant 3

Figure 9. RCADS Total Anxiety T-scores across baseline, treatment, and follow-up for participant 4

Figure 10. CBCL Internalizing T-scores across baseline, treatment, and follow-up for

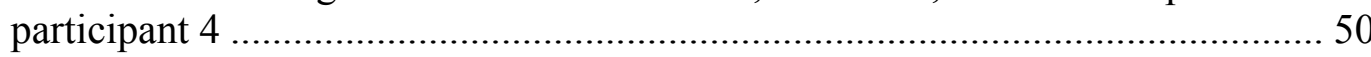

Figure 11. CBCL Anxiety T-scores across baseline, treatment, and follow-up for participant 4.

Figure 12. SDQ Overall Stress scores across baseline, treatment, and follow-up for participant 4

Figure 13. CIS total scale scores across baseline, treatment, and follow-up for participant 4

Figure 14. RCADS T-scores across baseline, treatment, and follow-up for participant 5 ... 57

Figure 15. SDQ Overall Stress scores across baseline, treatment, and follow-up for participant 5 
Figure 16. BIS total scale scores across baseline, treatment, and follow-up for participant 5

Figure 17. RCADS T-scores across baseline, treatment, and follow-up for participant 6 .... 61

Figure 18. SDQ Overall Stress scores across baseline, treatment, and follow-up for participant 6

Figure 19. BIS total scale scores across baseline, treatment, and follow-up for participant 6

Figure 20. RCADS T-scores across baseline, treatment, and follow-up for participant 7 .... 65

Figure 21. SDQ Overall Stress scores across baseline, treatment, and follow-up for participant 7

Figure 22. BIS total scale scores across baseline, treatment, and follow-up for participant 7

Figure 23. RCADS T-scores across baseline, treatment, and follow-up for participant 8 .... 69

Figure 24. SDQ Overall Stress scores across baseline, treatment, and follow-up for participant 8

Figure 25. BIS total scale scores across baseline, treatment, and follow-up for participant 8 


\begin{abstract}
ADAPTING EVIDENCE-BASED TREATMENTS FOR YOUTH IN A COMMUNITY MENTAL HEALTH SETTING: SINGLE CASE DESIGN
\end{abstract}

By: Alexis M. Quinoy, B.S.

A thesis submitted in partial fulfillment of the requirements for the degree of Master of Science at Virginia Commonwealth University.

Virginia Commonwealth University, 2011.

Director: Michael A. Southam-Gerow, Ph.D.

Associate Professor

Department of Psychology and Pediatrics

This single-case design study examined the effectiveness of adapting evidence-based treatments (EBTs) for children in a community clinic through a university-community partnership. Community clinic therapists treated eight youths (five males), ages 10 to 14 , of whom four were Caucasian, two were Latino, one was African-American, and one was Caucasian/African-American. Youths presented with a primary diagnosis of a DSM-IV (American Psychiatric Association, 1994) internalizing disorder (plus multiple comorbidities). An adapted treatment combining multiple elements based on two primary treatment manuals: Coping Cat (Kendall et al., 1990) and PASCET (Weisz et al., 1999) was used. Youths with comorbid externalizing symptoms were also treated with elements from a parent-training manual (Barkley, 1997). Results of visual and clinical significance analysis demonstrated mixed support for the adapted treatment in a community clinic setting. The 
findings support further efforts to test the effectiveness of adapted EBTs in a community clinic setting and population. 
Adapting Evidenced-Based Treatments For Youth in a Community Mental Health Setting: Single Case Design

Epidemiological data suggest a strong need for high quality mental health services to help the many youths with impairing mental health problems. According to the Surgeon General's Report on Mental Health, one in five children in the United States are affected by the signs and symptoms of a Diagnostic and Statistical Manual of Mental Disorders, Fourth Edition, Text Revision (DSM-IV-TR), mental health disorder over the course of one year (American Psychiatric Association [APA], 2000). Other epidemiological studies indicate that the overall prevalence of childhood mental health disorders ranges from 14 percent to 26 percent (Fergusson, Horwood, \& Lynskey, 1993; U.S. Public Health Service [Surgeon General], 2000; Hoagwood \& Olin, 2002).

Fortunately, there is a relatively strong and growing evidence base of child/adolescent mental health treatments (e.g., Chorpita \& Daleiden, 2009). Multiple efficacious treatments have been tested for many of the mental health problems which children experience, including anxiety, depression, and disruptive behavior disorders. Thus, there has been optimism in the field about transporting these treatments into multiple service settings. However, as will be discussed shortly, progress on the path to widespread dissemination has been inconsistent. The study proposed here represents an effort to identify methods to move the field further down the path toward a better integration of science and practice.

To place the proposed study in the context of the current state of the science, several literatures will be examined. First, the evidence bases for these three problem areas, given their commonness, are briefly and selectively reviewed. Next, I will discuss that despite the 
optimism, evidence from effectiveness trials has suggested that these evidence-based treatments (EBTs) do not always fare well as would be expected (e.g., Southam-Gerow et al., 2010; Weisz et al., 2009). Afterward, the many proposed barriers as to why these treatments have "struggled" in multiple service settings will be presented. Different ways for remedying this problem have been suggested; particularly partnership models have been proposed as a viable solution to aid in the implementation and dissemination of EBTs. Finally, I will describe the Chesterfield-VCU Adaptation of Depression and Anxiety Psychological Treatments for Youth (ADAPT) study, which implements a university-community partnership model in order to adapt EBTs in a public mental health context.

\section{Evidence-Based Treatment Review}

The following review provides an illustrative overview of the evidence-based treatment literature for youth internalizing (i.e., anxiety disorders, depressive disorders) and externalizing disorders (i.e., disruptive behavior disorders).

Internalizing disorders, such as anxiety and depression, are some of the most commonly diagnosed mental health problems that children and adolescents experience (Southam-Gerow \& Chorpita, 2007). Some studies suggest that anxiety symptoms and disorders may even be the most prevalent category, with up to 13 percent of children and adolescents suffering from at least one anxiety disorder (Silverman, Pina, \& Viswesvaran, 2008; Costello, Egger, Angold, 2005; Bernstein \& Borchardt, 1991). Somewhere between 6 percent (Fergusson et al., 1993) and 11 percent (McGee, Feehan, Williams, Partridge, Silva, $\&$ Kelly, 1990) suffer from mood disorders and lifetime rates for unipolar depression are approximately 20\% (Lewinsohn, Rohde, \& Seeley, 1998; Lewinsohn \& Essau, 2002;

Rudolph, Hammen, \& Daley, 2006). 
Anxiety disorders cause children and adolescents to feel frightened, distressed and worried for no apparent reason. Although most children and adolescents experience fears and worries, the fears and worries that are present in anxiety disorders actually impede daily activities or functioning (Christophersen \& Mortweet, 2001). Anxiety disorders include: separation anxiety disorder (SAD), panic disorder (PD), agoraphobia, generalized anxiety disorder (GAD), social phobia/social anxiety disorder, specific phobia, obsessive-compulsive disorder (OCD), posttraumatic stress disorder (PTSD), and acute stress disorder. Although the predominant feature of all these disorders is the emotion of anxiety, they are each distinguished by the focus of the child's anxiety (Southam-Gerow \& Chorpita, 2007). The evidence base for the treatment of childhood anxiety disorders is one of the largest and strongest in the child/adolescent treatment literature (Chorpita \& Southam-Gerow, 2006). Behavioral and Cognitive Behavioral Therapy (CBT) are the most studied and best supported treatments for helping youth who have been diagnosed with an anxiety disorder (Chorpita \& Southam-Gerow, 2006; Silverman et al., 2008). For example, CBT has been tested and found to be effective for anxiety disorders in youth in over 25 separate randomized trials (Silverman et al., 2008). Gradual exposures (in vivo or imaginary), coping plans, cognitive skills, relaxation, modeling, and psychoeducation comprise the main components of most CBT treatments (Silverman et al., 2008; Chorpita \& Daleiden, 2009). Most versions of behavioral therapy and CBT also include parental involvement, in some cases, involving the parents in all sessions with their child. Behavioral therapy and CBT can be administered in individual and group settings (Chorpita \& Southam-Gerow, 2006; Silverman, et al., 2008). They have also been delivered with good effects in schools, clinics, hospitals, day care centers, and even in homes; and evidence supporting CBT has been found across a variety of 
racial and ethnic groups, including Caucasian, African American, Hispanic/Latino, Asian, and Multiethnic (see Silverman et al., 2008).

Similarly to anxiety disorders, the youth mental health treatment literature also identifies many well-established EBTs for depressive disorders. Depression can cause feelings of hopelessness, guilt, and/or sadness in children and adolescents. Common symptoms of depressive disorders in youth include: sadness; decreased energy and interest in activities; irritability; changes in sleep and appetite; difficulty in thinking clearly, making decisions, and concentrating; lethargy and/or fidgetiness; and thoughts of death or suicide (DSM-IV-TR; APA, 2000). Depressive disorders increase in adolescence and rates for girls double those found for boys in adolescence (Costello, Erkanli, \& Angold, 2006; Angold, Erkanli, Silberg, Eaves, \& Costello, 2002). Primary depression diagnoses for youth include major depressive disorder (MDD) and dysthymia. The evidence base shows that there are well-established EBTs for MDD and dysthymia in youth, specifically CBT and interpersonal therapy (IPT) (see David-Ferdon \& Kaslow, 2008). In a recent review of treatments for youth depression, $\mathrm{CBT}$ was found to be efficacious in over 20 separate randomized trials (DavidFerdon \& Kaslow, 2008). CBT for depression focuses on identifying thoughts and behavioral patterns that lead to or maintain the problematic symptoms. For example, identifying negative beliefs, evaluating the evidence for these beliefs, and generating more realistic alternate thoughts. IPT for depression focuses on addressing the adolescent's interpersonal communication skills, interpersonal conflicts, and family relationship problems. Overall, the most common practice elements found in effective treatments for youth with depressed mood are cognitive skills, psychoeducation, maintenance, relapse prevention, activity scheduling, problem solving, and self-monitoring (Chorpita \& Daleiden, 2009). Research has indicated 
that treatment gains occurred, regardless of where treatment was provided (i.e., school, community clinics, primary care clinics, hospitals, or research settings) or regardless of how the treatment was delivered (i.e., group, individual, or family therapy). Age appears to moderate treatment effects, with studies showing treatments are more effective for older adolescents than for children or younger adolescents (see David-Ferdon \& Kaslow, 2008). There is also evidence that EBTs for youth depression might not be as efficacious for ethnic minority youth compared to Caucasian youth (e.g., Rohde et al, 2006; Cardemil et al., 2002).

Although internalizing disorders are common in children and adolescents, externalizing disorders like oppositional defiant disorder (ODD) and attention-deficit hyperactivity disorder (ADHD) represent the majority of referrals to mental health clinics (Loeber, Burke, Lahey, Winters, \& Zera, 2000). Externalizing disorders are shown to be present in about 5 percent to 10 percent of children (Fergusson et al., 1993; McGee et al., 1990; Costello, Mustillo, Erkanli, Keeler, \& Angold, 2003). Disruptive behavior disorders (DBDs) are defined by the presence of a persistent pattern of negative, defiant, or rule breaking behaviors that are disruptive to the youth's social, academic, familial, or personal functioning. DBDs include oppositional defiant disorder (ODD), and conduct disorder (CD). ODD is characterized by a pattern of negativistic, disobedient, and hostile behaviors, whereas, $\mathrm{CD}$ consists of more severe antisocial and aggressive behavior that may involve serious violations of other's personal rights (McMahon \& Frick, 2007). Attention deficit hyperactivity disorder (ADHD) is characterized by multiple symptoms of persistent and dysfunctional patterns of overactivity, impulsiveness, inattention and distractibility (Murphy, Cowan \& Sederer, 2001).

EBTs for DBDs and ADHD have also been identified (e.g. Eyberg, Nelson, \& Boggs, 
2008; see also Pelham \& Fabiano, 2008). In contrast to EBTs for internalizing disorders (i.e., anxiety, depression), which are usually child-focused and primarily follow the cognitivebehavioral theoretical approach, EBTs for externalizing disorders (i.e., ADHD, ODD, CD) apply social-learning and ecological systems theories, with greater focus on family and community involvement. For example, current research suggests that combined behavioral and pharmacological treatment is the most effective treatment for ADHD (Pelham \& Fabiano, 2008). Classroom management strategies and behavior parent training are considered the most efficacious of the behavioral approaches (see Pelham \& Fabiano, 2008). These programs use contingency management strategies (e.g., point/ token reward systems, time-out, response cost) to reinforce appropriate behavior and reduce unwanted behaviors. CBT programs for adolescents with ADHD may incorporate self-verbalization and problem solving strategies. Multimodal approaches that address functioning across multiple settings (e.g., home and school) have received the strongest support in the literature. For treatment of DBDs, behavioral parent management training has a strong evidence base (Eyberg et al., 2008). Research indicates that for adolescents, use of behavioral parent management training should be one component of an approach that would also include on (a) bolstering adolescents' coping skills and (b) strengthening the families' communication and problem solving skills (see Eyberg et al., 2008). Very few studies have included ethnic minorities, children from low SES families, and girls (Pelham \& Fabiano, 2008). Studies that have included these participants suggest that low-income or minority families respond not as well to behavioral treatments compared to Caucasian families; no gender effects have been found (Arnold et al., 2003). In conclusion, EBTs for externalizing disorders place greater emphasis on parent training and community involvement than EBTs for internalizing disorders. 


\section{Transporting EBTs into Community Settings}

The brief illustrative review demonstrates the promise of several psychosocial treatments for both internalizing and externalizing disorders leading to optimism that disseminating these efficacious treatments into multiple service settings such as community clinics would lead to improved outcomes for youths and families (e.g., Weisz et al., 1995). Unfortunately, despite the optimism, EBTs are not being consistently used in these settings (Norquist, Lebowitz, \& Hyman, 1999; Street, Niederehe, \& Lebowitz, 2000; Weiss, Catron \& Harris, 2000; Kazdin, 2000). Further, until recently, few efforts have been made to study the effectiveness of these treatments in these settings and of the few studies that have attempted, evidence has been mixed (Hoagwood \& Olin, 2002; Clarke, Hornbrook, Lynch, Polen, Gale, O’Connor, et al., 2002; Chorpita et al., 2002; Addis, Hatgis, Krasnow, Jacob, Bourne, \& Mansfield, 2004; Martin, Herie, Turner, \& Cunningham, 1998). In the next section, I will discuss important key terms used in dissemination research. Then, I will review those studies testing the effectiveness of EBTs in diverse community contexts.

The issue of efficacy versus effectiveness has become an integral part of psychotherapy literature in the past two decades. Although they often share a common goal of testing psychotherapy treatments in the hopes of increasing positive outcomes and functioning for people suffering from mental illness, they differ in many ways. According to Nathan, Stuart, \& Dolan (2000), efficacy studies maximize internal validity, are carefully controlled outcome studies, use homogenous patient samples, implement random assignment of participants, and, often, investigate manual-based, time-limited treatments. Efficacy studies also regularly take place in research settings. Once efficacy studies have provided supportive data, the treatment moves to the next stage: effectiveness studies (Southam- 
Gerow, Marder, \& Austin, 2008).

Effectiveness studies aim to be externally valid by generally testing treatments in realworld clinical settings. To maximize external validity and reflect real-world clinical settings, these studies are often less controlled, include more heterogeneous samples, and usually use non-random assignment. Effectiveness studies measure outcomes under conditions of high external validity and aim to determine whether treatments, which have been shown to be efficacious, are effective across broad populations and settings (Nathan et al., 2000). Once effectiveness studies have demonstrated positive outcomes outside of controlled research settings, an intermediate step between effectiveness and dissemination has been suggested (Southam-Gerow et al., 2008). Transportability studies focus on identifying certain key strategies needed to encourage the adoption and effective implementation of innovations (i.e., psychosocial treatments) in multiple settings. Implementation is described as a specific effort to make a program or treatment work across settings (e.g., community settings).

Dissemination, on the other hand, focuses on how a treatment is marketed after the means for implementing the treatment successfully have been identified (Southam-Gerow et al., 2008).

Recently in the youth psychotherapy literature, there has been an increased effort to implement EBTs for youth into community service settings. Here I look at the several ways researchers have attempted to examine the impact of both internalizing and externalizing disorder EBTs in non-research settings. For internalizing disorder EBTs, benchmarking studies, a meta-analysis of usual care, and effectiveness trials have contributed to the evidence base on dissemination efforts. For example, Weersing \& Weisz (2002) used a benchmarking strategy to compare usual care treatment for youth with depression in a community clinic to CBT clinical trial outcomes. A benchmarking strategy involves 
comparing outcome data obtained in a practice context with outcomes established for the same treatment in clinical trials. The community sample contained a higher proportion of ethnic minority youth and had a higher rate of comorbidity, similar to the findings in other studies, which showed differences between youth in community clinics versus researchbased settings (e.g., Southam-Gerow, Chorpita, Miller, \& Gleacher, 2008; Southam-Gerow, Weisz, \& Kendall, 2003). Results showed that the community youth sample outcomes more closely resembled treatment outcomes of youth in the randomized controlled trial's (RCTs) control conditions rather than the youth in the RCTs CBT conditions. Youth in the community usual care sample took twice as long to reach a similar level of symptom relief than the youth treated with CBT. This study showed that CBT for depression had a significant advantage over community usual care treatment, hence supporting the notion to increase dissemination efforts of EBTs into community service settings.

Other benchmarking studies have shown that similar outcomes can be obtained when EBTs are transported to community service settings versus controlled research trials. For example, Weersing, Iyengar, Kolko, Birmaher, \& Brent (2006) compared CBT treatment in an outpatient depression specialty clinic to a "gold standard" CBT RCT for depression (see Brent et al., 1997). The outpatient clinic sample had a higher rate of suicidality than the benchmark sample, as well as a higher rate of comorbidity. Results indicated that the outpatient youth outcomes were as favorable as the RCT, albeit the outpatient youths showed slower improvement. Comorbidity or suicidality were not significant factors in treatment outcomes indicating the robustness of the treatment across complicating factors. Another benchmarking study that compared school based CBT for depression to results from prior efficacy trials of the same treatment also showed support for the transportability of EBTs into 
community service settings. Shirk, Kaplinksi, \& Gudmundsen (2008) found that school based CBT outcomes were comparable to the results in efficacy trials and actually better than an efficacy trial with the original manual and a prior school based CBT trial. Positive outcomes were robust across age, gender, and ethnic groups as well as with youth with comorbid symptomatology. Overall, benchmarking studies have indicated positive outcomes for EBTs in community settings above usual care treatment, supporting recent transportability and implementation efforts.

Other studies have also shown support for transporting EBTs into non-research based settings. A meta-analysis of 32 randomized trials that compared EBTs to usual care for youth therapies found that EBTs outperformed usual care (Weisz, Jensen-Doss, \& Hawley, 2006). Results indicated a mean effect size of .30 , which falls within the range of small to medium effects (Cohen, 1988), and effect size was not reduced by high levels of severe symptomatology or by inclusion of ethnic minority youths. However, five studies included in the meta-analysis indicated that usual care was better than EBTs. The meta-analysis gives support for the dissemination of EBTs, but the authors suggest that effect sizes could be larger if the treatments were strengthened by necessary adaptations.

Although the benchmarking studies and the meta-analysis show positive support for recent dissemination efforts, some effectiveness studies have shown no differences between EBTs and usual care. In 2005, Barrington and colleagues tested the effectiveness of cognitive-behavioral therapy (CBT) for childhood anxiety disorders against usual care in a community clinic setting using a randomized controlled trial (RCT) design. Participants $(N=54)$ ages 7 to 14 years old were randomly assigned to either the CBT group $(n=28)$ or the usual care group $(n=26)$. No significant group differences were found on 
any measure and children in both groups improved over time. Southam-Gerow and colleagues (2010) also found no significant differences on symptom or diagnostic outcomes for a CBT treatment condition for childhood anxiety disorders versus usual care in several community clinics. Overall, the benchmarking studies support the transportability of EBTs, but these positives are not as clearly demonstrated in the meta-analysis (e.g. lower effect sizes) and the effectiveness trials.

Studies comparing CBT for youth depression have also shown mixed evidence. For example, a recent RCT that compared CBT for depression to usual care in a community clinic found that the two treatment conditions did not differ in outcomes (Weisz et al., 2009). However, compared to usual care, CBT was briefer, superior in parent engagement, youth were less likely to require additional services, and it was less costly. Kerfoot, Harrington, Harrington, Rogers, and Verduyn (2004) studied the effectiveness of brief CBT for depressed adolescents delivered by social workers in the United Kingdom. Brief CBT was compared to routine care and no significant differences were found between the two groups on symptom and functioning outcome measures.

Similarly to EBTs for internalizing disorders, effectiveness studies have also been used to test the applicability of EBTs for externalizing disorders in other service settings. According to Evans (2005) the "availability of cost-effective programs for children with ADHD in school settings has been well documented." Due to the nature of externalizing disorder EBTs, many of them have initially been tested with effectiveness-type designs, making the move to dissemination smoother than internalizing disorder EBTs. Behavioral contingency management is already widely being used in school settings (Gottfredson \& Gottfredson, 2001; Walker et al., 2003), and multisystemic therapy (MST; Henggeler, 
Schoenwald, Borduin, Rowland, \& Cunningham, 1998) programs have been well established in 32 states and 10 nations, hence supporting transportability efforts (Schoenwald, 2010). In this section, I will review specific externalizing disorder EBT programs that have shown positive outcomes in other service settings and are well established in the treatment literature. Multidimensional Treatment Foster Care (MTFC) is an example of an EBT for youth with severe and chronic delinquent behavior that has supported effectiveness in community settings (Eyberg et al., 2008). MTFC is a community-based program designed as an alternative to institutional-, residential-, and group-care placements. Two studies have found MTFC superior to usual group home care for adolescents with histories of chronic delinquency (Chamberlain \& Reid, 1998; Leve, Chamberlain, \& Reid, 2005).

Another example is MST, an intervention approach for treating adolescents with serious antisocial and delinquent behavior (Henggeler et al., 2009). It combines treatments and procedures as needed to provide an intensive family and community-based intervention designed for the individual family, with the goal of promoting responsible behavior and preventing need for out-of-home placement (Eyberg et al., 2008). Studies with adolescents who committed criminal offenses found MST superior to usual community services as well as alternative community treatments (Borduin et al., 1995; Henggeler, Melton, \& Smith, 1992; Henggeler, Melton, Brondino, Scherer, \& Hanley, 1997). In conclusion, effectiveness studies for externalizing disorder EBTs have shown success when transported into other services settings.

Altogether, support for the potency of EBTs in community service settings has been mixed. Overall, evidence for the transportability of externalizing disorder EBTs has shown positive outcomes, and EBT programs have been well established in many diverse service 
settings. On the other hand, evidence for the transportability of internalizing disorder EBTs is not as clear; benchmarking studies support positive outcomes for internalizing EBTs, but these outcomes are not as clearly shown in the meta-analysis and the effectiveness trials.

\section{Challenges to Transportability}

A myriad of reasons have been identified to explain the science-practice gap and these mixed findings (Southam-Gerow, 2004; Weisz, 2000). Some have suggested that the research-based method of treatment development does not take into account the complexity of most community-based service clinic settings. In order to capture the complex nature of these settings, Schoenwald and Hoagwood's model provides multiple levels of variables to consider when developing and adapting treatments; these levels include: (1) client level factors, (2) provider level factors, (3) clinic/agency level factors, and (4) service system level factors (2001). Considering these unique factors when adapting EBTs, will allow us to identify necessary adaptations at each level in order to improve transportability into a community service setting. For the purposes of the present study, I examine two of these in detail: (a) client level differences, and (b) therapist level differences.

\section{Client Level Differences}

One potential obstacle of transporting EBTs into community service clinics is related to a mismatch between the complexity of child problems and the focus of many EBTs. Development and testing of these efficacious treatments for children with mental health disorders has been conducted in university-based research settings, which may be different from community clinics. Many of the treatments are developed for children and adolescents that have a single DSM-IV diagnosis or at least the treatments focus on a single disorder or problem type. In contrast, many youth seen in community service clinic settings meet criteria 
for more than one DSM-IV diagnosis (Southam-Gerow, Weisz, \& Kendall, 2003; Bird, Gould, \& Staghezza, 1993). Anxiety clinic research samples have been shown to be very similar across different settings, supporting the homogeneity of the populations used to study and test these treatments (Southam-Gerow et al., 2006). However, recent evidence has shown that there are a number of differences between clients seen in research versus service clinic settings that may affect the applicability of treatments in a community outpatient service clinic (Kazdin \& Whitley, 2006; Schoenwald \& Hoagwood, 2001). For example, children with internalizing disorders in community mental health clinics have significantly higher levels of externalizing comorbidity as compared to those seen in research clinic settings (Southam-Gerow et al., 2003). The focus on youth-only involvement for internalizing disorder EBTs may present a problem for the effectiveness of these interventions in service settings. Comorbidity is one of many complex factors that occur in clinic referrals (Kazdin, 1995). Fewer than $33 \%$ of children who are diagnosed with a depressive disorder solely experience depression; most of these children are diagnosed with another disorder, most commonly anxiety disorders or a disruptive behavior disorder (Angold \& Costello, 1993; Hammen \& Rudolph, 2003). Also, studies have shown that children with comorbid disorders show greater impairment at home, at school and in social settings (Kazdin \& Whitley, 2006).

Studies have found that comorbidity may not be relevant to treatment outcomes and may not limit the applicability of these treatments in service settings (Kazdin \& Whitley, 2006; Kendall, Brady, \& Verduin, 2001; Southam-Gerow, Kendall, \& Weersing, 2001). In contrast, Southam-Gerow and colleagues (2003) found that the comorbidity rates in these aforementioned study samples were different from those found in typical service clinics. Since many children in service settings present with comorbid externalizing 
symptomatology, transportability of EBTs for internalizing disorders into these settings will be difficult due to the theoretical and structural differences (e.g. parental and community involvement) between internalizing disorder EBTs and externalizing disorder EBTs. Altogether, EBTs for internalizing disorders may need to be adapted and integrated into a more socio-ecological framework to improve chances of successful outcomes with youth experiencing externalizing symptomatology. Once adaptations occur, researchers may more easily move from efficacy research in research-based settings to seeing how these treatments function effectively in service settings.

Clients also appear to differ systematically between research and service contexts such that youths seen in service clinics possess multiple sociodemographic disadvantages, compared to youths seen in research clinics (Southam-Gerow et al., 2008; Southam-Gerow et al., 2003; Ehrenreich, Southam-Gerow, Hourigan, Wright, Pincus, \& Weisz, in press). For example, a recent study found that public clinic youth were more likely to be a minority, have a lower socioeconomic status, and come from single-parent families (Southam-Gerow et al., 2003). Research has shown that practitioners in community clinics indicate that clients they see in these clinical settings have more severe symptomatology, more complex cases, and usually carry more than one diagnosis (Dulcan, 2005; Westen, Novotny, \& ThompsonBrenner, 2004; Kazdin \& Whitley, 2006). Due to these differences seen in community clients, including a youth's family as part of treatment is a reality in a community practice setting and many EBTs do not include parental involvement (Surgeon General, 2000; Kazdin $\&$ Whitley, 2006). In addition, differences in terms of therapist characteristics may also be present, further undermining the notion that the EBTs will be easily applied in diverse settings (e.g., Schoenwald \& Hoagwood, 2001; Southam-Gerow, Hourigan, \& Allin, 2009). 


\section{Therapist Level Differences}

Therapist level differences may also complicate efforts. For example, community service clinics employ a wider range of mental health providers (e.g., masters $\&$ PhD-level psychologists, bachelors \& masters-level social workers, psychiatrists, nurses), with diverse educational training backgrounds that may not include training in evidenced-based practices (Smith-Boydston \& Nelson, 2008; Addis \& Krasnow, 2000). Most EBTs that have been developed in research settings are based in behavioral or cognitive-behavioral theory (CBT), whereas non-behavioral theoretical models (e.g., psychodynamic, family systems) have often guided treatment in community service settings (Weisz \& Kazdin, 2003). Providers in research settings are also different than providers in community settings because the former are usually trained in a single protocol for one specific problem in the context of a federallyfunded research study, and see only those clients that meet certain criteria for the specific manual based treatment (Addis \& Krasnow, 2000). Community providers on the other hand, are usually not trained on a specific manual and may use many different treatment techniques when interacting with youths and their families (Weisz et al., 2003).

Due to these differing characteristics in education and training, many community mental health providers appear to have difficulty "buying in" to EBTs (Weisz et al., 2000). Community providers are at times critical of "cookbook" style manuals and feel that clinical judgment is a more appropriate tool for positive treatment outcomes (Levant, 2004; Dulcan, 2005; APA Policy Statement on EBPP, 2005). Providers also feel that EBTs are less appropriate for their heterogeneous caseloads, which make it difficult to find manuals that meet the needs of their clinical populations (Henggeler et al., 1995; Chorpita \& Donkervoet, 2005). Research has also shown that there are few incentives for community practitioners to 
learn new approaches, further increasing the difficulty to want to "buy in" to using EBTs in their clinical practice (Weisz et al., 2000). Hence, transportability and implementation efforts that do not consider differences in therapist characteristics between research-based settings and community clinics may have a more difficult time transporting EBTs in these settings.

Overall, there are a number of factors complicating the transportability of EBTs from research clinics to other contexts and we need to identify pathways to overcoming them. The current study represents one possible pathway to transportability and implementation, the use of a partnership model. In the next section, I will briefly identify the different ways that have been proposed as pathways of transportability and implementation, focusing on partnership models, before describing the current study.

\section{Solutions to Challenges}

Efforts to test EBTs in diverse settings have been mixed, transporting EBTs directly from a research-based setting to a community service setting has not worked well, and thus attention to implementation methods that could improve transportability is warranted. Given the difficulties in transporting EBTs, some have advocated an intermediate step between (a) development of an efficacious treatment and (b) wide-spread dissemination (e.g., Schoenwald \& Hoagwood, 2001). Different implementation techniques have been identified to guide transportability efforts into community settings (Schoenwald \& Hoagwood, 2001; Southam-Gerow et al., 2008). For example, Weisz (2000) proposed the clinic-based treatment development (CBTD) model as a way to move EBTs from efficacy studies to effectiveness studies. CBTD sequences effectiveness studies to increasingly include populations, clinicians, and clinical settings of usual care circumstances (Schoenwald \& Hoagwood, 2001). The clinic intervention development (CID) model adds onto CBTD by 
also including practice setting variables (Hoagwood, Burns, \& Weisz, 2002). Other implementation techniques have also been proposed to guide transportability efforts (see Southam-Gerow et al., 2008). In this next section, I will focus on the partnership model framework and discuss a university-community partnership for treatment adaptation.

\section{A Partnership Approach to Treatment Adaptation}

One way proposed to implement EBTs and bridge the gap between science and practice is to develop and test EBTs in collaboration with community mental health clinics and adapt them in order to fit the needs of the client population in the community setting and treatment providers in the service clinic (Southam-Gerow et al., 2008). According to this perspective, the adaptation of these treatments will require greater levels of collaboration between researchers and community mental health stakeholders (Southam-Gerow, 2005). Models emphasizing collaborative relationships between researchers and community stakeholders have been in use for many years in fields such as education (e.g., Adelman \& Taylor, 2004) and public health (e.g., Harper et al., 2004; Sullivan \& Kelly, 2001); and more recently, mental health researchers have begun to use partnership models as a way to implement EBTs (Gotham, 2004; Hoagwood et al., 2002).

Participatory action research (PAR; Jason, Keys, Suarez-Balcazar, Taylor, \& Davis, 2004) was the partnership framework model used to aid the transportability and implement EBTs into a community mental health setting in the current study. PAR is a diverse set of strategies designed to empower and give voice to a group or groups of citizens, and to ultimately create social action (Taylor et al., 2004). The model involves research participants and consumers as collaborative partners in the decision-making process and seeks to create change at an organizational or systemic level. PAR allows all involved parties to have a say 
(Middlestadt et al., 1997). Community stakeholder involvement is a key element in this framework, and our study aims to make this an important focus in the adaptive treatment process. The PAR framework will help us to find solutions to many of these proposed barriers (e.g., client- and therapist level differences) and allow us to successfully adapt and test a new treatment for child mental health problems in a community service setting.

\section{University-Community Partnership Approach}

The present study is one component in a larger research project, the ChesterfieldVCU Adaptation of Depression and Anxiety Psychological Treatments for Youth (ADAPT) study. ADAPT was designed to provide a preliminary test of the application of a partnership model in adapting EBTs in public mental health contexts. ADAPT exemplifies a universitycommunity partnership approach for treatment adaptation in the youth treatment literature (e.g., Southam-Gerow et al., 2009), and is a collaborative project aimed at adapting and implementing EBTs designed for youth with internalizing problems (i.e., anxiety and/or depression) and comorbid externalizing behavior problems for use in a public mental health setting. As stated previously, the presence of multiple, comorbid disorders may complicate transportability and implementation of EBTs because most treatment programs are designed to focus on a single problem. The ADAPT study, through a series of phases, attempts to develop and test a method for implementing EBTs for youth with multiple, impairing problems.

ADAPT is a mixed quantitative/qualitative project that consists of four phases: (a) preliminary focus groups, (b) single case series, (c) open trial, and (d) post-project focus groups. The current study draws on data from the second phase: the single-case series. The single-case series was designed as an initial step in adapting EBTs for internalizing disorders 
(i.e., CBT) to address youth with comorbid externalizing symptomatology. In this sense, the single case phase was a small-scale transportability study since it aims, through treatment adaptation and implementation of the partnership model, to encourage the adoption of this treatment into a community setting. The study involved working in partnership with therapists to apply EBTs to cases identified to fit a particular profile (i.e., youth with internalizing and externalizing disorders). Although treatment for the youth was planned to follow EBTs, there was dialogue between researcher and the partner therapists to adapt and adjust the EBTs in response to therapist judgments. The goal of the single case series was to (a) demonstrate that adapted EBTs would lead to improvements in client functioning and (b) identify ways to formalize the adaptations into manual-like format for the third phase of the project.

\section{Justification of Single Case Design}

Single case design was selected for the second phase of ADAPT because it will allow us to establish data on safety of our early attempts to adapt EBTs for youth with comorbid disorders, as well as provide preliminary evidence of positive effects, and aid in further adaptation of the treatment (Photos, Michel, \& Nock, 2008; Southam-Gerow et al., 2009). Single-case design is appropriate for analyzing initial feasibility data (Nock et al., 2008). Although single case design has been used less often in the recent treatment literature, Kazdin (1982) states that it may be most relevant in treatment research because it allows for the observation of clinical change in an individual subject. It can also allow the researcher to see treatment differences in individuals that might get lost in tests of group differences (Sidman, 1960). 
Although single-case design is limited in the validity of references, threats to validity can be ruled out through repeated assessment. In our study, a multiple baseline design was used in order to establish a consistent baseline in the dependent variable before beginning treatment (Nock et al., 2008). In this case, threats to validity (e.g., regression to the mean, history, maturation) can be controlled and inferences about changes in data points as related to treatment progress can be made with greater confidence.

Visual inspection of graphed data points is the most commonly used and conservative method to analyze data in single-case design, relying on large changes in the measured data (Gaynor, Baird, \& Nelson-Gray, 1999; Kazdin, 1982). Following a stable baseline measurement period, a researcher can identify changes in levels or trends of the data. Clinical significance analysis is another means of examining data in single-case design. Here normative data for the dependent measures is used as a base of comparison for subject scores (Kendall \& Grove, 1988). A subject can be classified as treatment success/failure based on their status in comparison to normative data and if they are no longer in the dysfunctional range of scores after treatment. An outcome measure's sensitivity to change, the degree to which a measure is likely to reflect changes that occur as a result of the intervention, is important to consider when identifying changes in trends of data (McClendon et al., 2011). Especially in cases when a participant's scores never reach the dysfunctional range or when the change is not large enough to be clear. Reliable change index (RCI) is one way of identifying meaningful change in a participant's data (Jacobson \& Truax, 1991). RCI indicates whether change reflects actual clinical change on a measure as opposed to normal fluctuations in scores over time or measurement error. Using the RCI and the clinical cutoff cases can be categorized as: (1) recovered (passed clinical cutoff and RCI criteria), (2) 
improved (passed RCI criteria but not clinical cutoff), (3) unchanged (passed neither criteria), and (4) deteriorated (passed RCI criteria, but towards a worsening direction).

The proposed project applies multiple baseline single case design methods to test adapted EBTs for youth with internalizing disorders and comorbid externalizing symptomatology with a small sample of children $(n=8)$ from a community mental health clinic. Throughout treatment, assessments will be conducted across three phases: (a) Baseline, (b) Treatment, and (c) Follow-up. Both parent-report and youth self-report measures will be administered. Therapists in the community clinic who have been trained to use the adapted treatment will administer treatment.

It is hypothesized that treatment gains will be achieved for each individual enrolled in the single case series across parent and child reporters on both symptom and diagnostic measures for both internalizing and externalizing domains. It is also hypothesized that functioning will increase for each individual on global functioning measures indicating decreased impairment. The results of this single-case series will also add to the growing evidence base of the effectiveness of EBTs for youth in community service settings, and further support the use of partnership models to improve further implementation of mental health treatments in multiple service settings.

\section{Methods}

\section{Participants \& Screening Procedure}

This study involved the participation of eight $(n=8)$ children (between the ages of 8 and 14) and their families. Participants were recruited for the study from the outpatient referral pool of a community mental health clinic in a metropolitan area in the mid-Atlantic region of the United States. After an initial phone screening, families with children between 
the ages of 7 to 16 who presented to the clinic with a problem consistent with a $D S M-I V-T R$ (APA, 2000) diagnosis of Major or Minor Depressive Disorder, Dysthymic Disorder, Generalized Anxiety Disorder, Separation Anxiety Disorder, or Social Phobia were invited to an initial assessment. Effort was made to select participants such that an equal number had either a primary anxiety disorder or a depression disorder; in the end three children had an anxiety disorder as their primary target problem, and five children had a primary depressive disorder. Inclusion criteria for the study included: (a) the presence of one of the target disorders that merited treatment priority after the initial assessment and (b) presence of symptoms of either Oppositional Defiant or Conduct Disorder. Children with a diagnosis or symptoms of Attention Deficit/Hyperactivity Disorder (ADHD) were included if they were stabilized on an ADHD medication. Participants were excluded if they were currently taking any psychotropic medications (except for ADHD medication), and/or diagnosed with a Pervasive Developmental Disorder, Mental Retardation, or a psychotic disorder.

Twelve families completed the first baseline assessment. Of these 12 families, three families did not meet inclusion criteria and two families were lost to follow up. There were 7 total families who completed treatment and at least one follow-up assessment. Participant 1 was lost to follow-up, and due to a lack of data for comparison, this participant will not be included in the analyses. No adverse events were reported. See Table 1 for participant information.

Table 1.

Participant Demographics

\begin{tabular}{|l|c|c|c|c|c|c|c|}
\hline $\begin{array}{l}\text { Participant } \\
\text { Number }\end{array}$ & Sex & $\begin{array}{c}\text { Age at } \\
\text { Intake }\end{array}$ & Ethnicity & $\begin{array}{c}\text { Intake } \\
\text { Diagnosis }\end{array}$ & $\begin{array}{c}\text { \# of Baseline } \\
\text { Assessments }\end{array}$ & $\begin{array}{c}\text { \# of } \\
\text { Treatment } \\
\text { Assessments }\end{array}$ & $\begin{array}{c}\text { \# of Follow- } \\
\text { Up } \\
\text { Assessments }\end{array}$ \\
\hline
\end{tabular}




\begin{tabular}{|c|c|c|c|c|c|c|c|}
\hline \multicolumn{8}{|c|}{ Table 1 (continued) } \\
\hline \multicolumn{7}{|c|}{\begin{tabular}{c|c|} 
Caucasian/ & Minor \\
African- & Depression, \\
American & Encopresis, \\
& ADHD
\end{tabular}} & 0 \\
\hline 2 & Male & 12 & Caucasian & $\begin{array}{l}\text { Social } \\
\text { Phobia }\end{array}$ & 3 & 3 & 2 \\
\hline 3 & Male & 10 & Caucasian & \begin{tabular}{|c|} 
Minor \\
Depression, \\
ADHD, \\
ODD
\end{tabular} & 5 & 5 & 2 \\
\hline 4 & Male & 12 & Caucasian & $\begin{array}{c}\text { Panic } \\
\text { Disorder, } \\
\text { Separation } \\
\text { Anxiety } \\
\text { Disorder }\end{array}$ & 3 & 2 & 3 \\
\hline 5 & Female & 14 & Latino & \begin{tabular}{c|} 
Minor \\
Depression
\end{tabular} & 4 & 2 & 3 \\
\hline 6 & Male & 11 & Caucasian & \begin{tabular}{|c|} 
Minor \\
Depression, \\
Enuresis, \\
ADHD
\end{tabular} & 3 & 3 & 3 \\
\hline 7 & Female & 12 & $\begin{array}{l}\text { African- } \\
\text { American }\end{array}$ & $\begin{array}{c}\text { Simple } \\
\text { Phobia, } \\
\text { Social } \\
\text { Phobia, } \\
\text { GAD }\end{array}$ & 3 & 1 & 4 \\
\hline 8 & Female & 14 & Latino & $\begin{array}{c}\text { Major } \\
\text { Depression }\end{array}$ & 1 & 1 & 1 \\
\hline
\end{tabular}

\section{Measures}

Measures were chosen based on the assessment model proposed by Hoagwood et al. (1996) that focuses on a broad range of possible treatment effects, including five domains of outcomes: (a) symptoms and diagnoses, (b) functioning, (c) consumer perspectives, (d) 
environments, and (e) systems. For the present study, I focus on two domains (symptoms and functioning) because they are most relevant to the proposed goals of the study. See Table 2 for single case series data collection schedule.

\section{Symptoms and diagnosis domain.}

\section{Schedule for Affective Disorders and Schizophrenia for School-Age Children-}

Present and Lifetime Version (K-SADS-PL). (Kaufman, Birmaher, Brent, Rao, \& Ryan, 1997). The K-SADS-PL is a semi-structured diagnostic interview that includes a screen interview and five diagnostic sections. The 82-item screen interview is used to eliminate the longer full diagnostic sections for those children that do not receive a threshold score on the screen interview. The diagnostic sections include (a) affective disorders, (b) psychotic disorders, (c) anxiety disorders, (d) behavioral disorders, and (e) substance abuse, eating, and tic disorders. Retest reliability across three weeks ranged from .63 to 1.00 for present diagnoses and from .55 to 1.00 for lifetime diagnoses (Kaufman et al., 1997). Interrater reliability was high ranging from .93 to 1.00 and concurrent validity was also fair to good across diagnostic categories. Psychometric data compare favorably with those reported for other diagnostic interviews (e.g., DISC, ADIS-C). Trained clinical psychology doctoral students administered the K-SADS-PL at intake and follow-up. Only parent report data was used to determine diagnosis at baseline and follow-up.

Child Behavior Checklist (CBCL). (Achenbach \& Rescorla, 2001). Caregivers completed the CBCL, a widely used and extensively researched measure of demonstrated reliability and validity (see Achenbach \& Rescorla, 2001). The CBCL's 118-item problem portion is used to assess a broad range of symptoms. It yields scores for broadband internalizing and externalizing scales, as well as more focused narrow-band scales (e.g., 
anxiety). Reliability for the CBCL is excellent with alphas ranging from .78 to .97 and retest reliability coefficients above .72. Achenbach \& Rescorla recommend a one to two month interval between administrations (2004). It has been suggested that the CBCLs limited response range (i.e., not true, sometimes true, and often true) may affect the CBCLs sensitivity to change (Lipsey, 1990). A recent study indicated a broadband problems scale (i.e. Total Problems, Internalizing Problems, and Externalizing Problems) reliable change index (RCI) of 4.8 points as indicative of reliable clinical improvement (McClendon et al., 2011).

\section{Revised Child Anxiety and Depression Scale - Youth and Parent Versions} (RCADS) (Chorpita, Yim, Moffitt, Umemoto, \& Francis, 2000). The RCADS is a 47-item child self-report measure that assesses symptoms of several DSM-IV-TR anxiety and depressive disorders. The measure yields 6 factors (i.e., separation anxiety disorder, social phobia, obsessive-compulsive disorder, panic disorder, generalized anxiety disorder, and major depressive disorder) and 2 total scores. The RCADS has a strong psychometric profile including alphas ranging between .71 and .85 and one-week retest reliability coefficients ranging from .65 to .80 . In addition, the RCADS depression scale correlated highly (.70) with the Children's Depression Inventory whereas the several anxiety scales were correlated highly with the Revised Child Manifest Anxiety Scale (e.g., de Ross, Gullone, \& Chorpita, 2002). There is currently no information in the literature on a specific reliable change index for the RCADS.

Strengths and Difficulties Questionnaire (SDQ) (Goodman, 2001). The SDQ is a brief ( 25 items) parent report behavioral screening measure that can be used for children ages 3-17 years of age. Norms for children in the United States were gleaned from the National 
Health Interview Study, which included a sample of 9,878 children. The SDQ has 5 subscales (five items per subscale) for internalizing and externalizing behaviors (Emotional Distress, Conduct Problems, Hyperactivity, Peer Problems, and Prosocial) and a total Overall Stress scale. Overall Stress scale scores on the Symptoms and Difficulties Questionnaire (SDQ) from 14 to 16 are considered "borderline" while those scores above 17 are considered to indicate "abnormal" levels of overall stress (Goodman, 2001). Retest reliability .62 and internal reliability estimates are above .70 . Although no reliable change index exists for the SDQ, the SDQ has been shown to successfully differentiate between youth who were identified as having a disorder with those who were not (Goodman, 2001).

\section{Functioning domain.}

Columbia Impairment Scale (CIS) (Bird et al., 1993). This is a 13-item scale designed to provide a global measure of psychosocial impairment based on parent report. The scale was designed to assess four major areas of functioning: interpersonal relations, certain broad areas of psychopathology, functioning at school or work, and use of leisure time. Items are scored on a Likert scale ranging from 0 (no problem) to 4 (a very big problem); the potential total score ranges 0 to 52 . A score of 16 or greater is considered indicative of definite impairment (Bird et al., 1993); thus, higher scores indicate greater levels of impairment. It has been demonstrated that the CIS provides an adequately reliable and valid measure of impairment and also correlates highly with the clinician determined scores of the Children's Global Assessment Scale (Shaffer et al., 1983; Bird et al., 1993). Internal consistency reliability ranges from .85 to .89 and test retest reliability (mean $=14.7$ days) is .89 . 
Brief Impairment Scale (BIS) (Bird et al., 2005). This is a 23-item scale that provides a parental report of their child's functioning across the following domains: interpersonal relations, school/work, and self-care/self-fulfillment. A score of 14 or greater is considered indicative of definite impairment (Bird et al., 2005); thus higher scores indicate greater levels of impairment. The measure has demonstrated high convergent and concurrent validity. Internal consistency scores range from .81 to .88 and from .56 to .81 on the three subscales and test-retest reliability coefficients around .70 .

Table 2.

Measurement Battery Composition at Each Assessment Point

\begin{tabular}{|l|c|c|c|c|}
\hline Measures & $\begin{array}{c}\text { Baseline } \\
\text { Assessment } 1 \\
\text { (Intake) }\end{array}$ & $\begin{array}{c}\text { Baseline } \\
\text { Assessments 2-4 }\end{array}$ & $\begin{array}{c}\text { Treatment } \\
\text { Assessments }\end{array}$ & $\begin{array}{c}\text { Follow-up } \\
\text { Assessments 1-3 }\end{array}$ \\
\hline$K-S A D S$ & Collected & & Collected \\
\hline$C B C L$ & Collected & Collected & Collected & Collected \\
\hline$R C A D S$ & Collected & Collected & Collected & Collected \\
\hline$S D Q$ & Collected & Collected & Collected & Collected \\
\hline$C I S$ & Collected & Collected & Collected & Collected \\
\hline$B I S$ & Collected & Collected & Collected & Collected \\
\hline
\end{tabular}

\section{Procedures}

The clinic's intake manager informed children and families from the clinic's outpatient referral pool, between the ages of 7 and 16, about the study. Interested families were asked for permission to provide the researchers with name and telephone only. Virginia Commonwealth University research staff screened families for interest and possible fit with study inclusion criteria. Children with a primary referral problem related to anxiety and/or depression with externalizing symptomatology were invited to an initial intake session.

Children and families were consented and assented and a battery of tests was administered 
(see Table 1). If inclusion criteria were met, participants were officially enrolled in the study. Participants received a $\$ 35$ gift card for the first assessment and then a \$20 gift card for the subsequent assessment meetings. The study is approved by the Virginia Commonwealth University (VCU) Institutional Review Board.

\section{Assessments}

Single-case research design involves multiple assessments before, during, and after an intervention (Kazdin, 1982). In the case of the present study, assessments were conducted during a baseline period, during treatment, and after follow up. To determine the length of the baseline period for each participant, we employed a randomization procedure. Methodologists have recommended this procedure to strengthen single-case design (Koehler \& Levin, 1998). Randomization will allow for systematic staggered introduction of the treatment phase across youths, with the goal of demonstrating effect produced during the targeted phase while controlling for other variables (e.g., history) (Koehler \& Levin, 1998). Therefore, the number of baseline assessments was determined randomly using a random number generation program. It has been suggested that in order to stabilize the baseline before introduction of the treatment phase a data based baseline determination procedure should be used (Koehler \& Levin, 1998; Kazdin, 1982). Unfortunately, due to clinic regulations, youth could not wait more than a month to begin treatment and therefore we could not continue to administer baseline assessment until a stable pattern of outcomes was produced. Overall, the study was designed so that each family would receive at least 6 assessments across the three phases. Assessments were spaced out based on feedback from families and therapists about what might work best. 
Baseline phase. Each participant had between 1 to 5 assessments in the baseline phase, including the initial meeting; the number for each family was determined randomly at the conclusion of the first assessment. The number of assessments was constrained to be at least 3 and no more than 5. The number of baseline assessments was also affected by the availability of the families to come into the clinic.

Treatment phase. Treatment was limited to 20 sessions based on feedback from families and therapists about what might work best. The treatment phase lasted the entire course of treatment and assessments occurred every six weeks. Similar to the baseline phase, treatment assessments were affected by the availability of the families to come into the clinic. This is also true of the follow-up assessments.

Follow-up phase. This phase consisted of assessments every eight weeks. All families were assessed as soon as possible after the last treatment session, which counted as the first follow-up assessment. The total number of follow-up assessments was determined by the number of previous assessments in the baseline and treatment phases ( 1 to 3 total).

\section{Treatment}

The treatment used in the study varied by participant and was based on two primary treatment manuals: Coping Cat and PASCET. The Coping Cat program is an individualbased cognitive-behavioral treatment (ICBT) for youth anxiety disorders (Kendall, Kane, Howard, \& Siqueland, 1990). Coping Cat is a 16- to 20 -session manual-based treatment program that involves a skills training component and an exposure component. The PASCET program (Weisz, Moore, Southam-Gerow, Weersing, Valeri \& McCarty, 1999) is a 15session, manualized, cognitive-behavioral treatment program focused on treating children with depressive disorders (Weisz, Southam-Gerow, Gordis, \& Connor-Smith, 2003). The 
PASCET program centers on helping youth control their mood through strengthening of primary and secondary control and coping skills. For cases where conduct problems were present and interfering, a parent-training manual was also employed (Barkley, 1997). These three manuals were chosen for the study because they have large evidence bases that support their use as well-established efficacious treatments for children and adolescents and they have also been tested or are being tested in community settings (Weisz et al., 2009). In the next section, I will describe the different treatment elements used in the study.

\section{Elements.}

Psychoeducation is a key treatment component where an individual is provided information about the nature of their presenting problem or about general psychological principles. For this study, psychoeducation was tailored towards a participant's specific diagnostic profile. Emotion education involves identifying individualized somatic reactions to distress so that a youth can begin to implement a plan for coping with their problem. Exposure is a key component of cognitive-behavioral treatment for anxiety in which repeated contact to a stimulus that is feared but safe leads to a reduction or elimination of the fear response. A fear ladder, is part of exposure, and helps a youth identify a feared stimulus and create a list with an array of ways to expose the youth to the feared stimulus. In cognitive restructuring, an individual is taught to identify and challenge distorted or inaccurate thinking and then work to develop a more realistic belief system. Self-monitoring helps a youth attend to their thoughts, feelings, and behaviors and provides essential feedback about the changes that need to be made and how well the process is going. Relaxation strategies involve teaching an individual a method for self-calming which may include breathing retraining and/or progressive muscle relaxation. Breathing retraining is where youth are 
taught to breathe more deeply and from his/her diaphragm. Progressive muscle relaxation involves teaching youth to tense and relax different muscle groups, which helps a youth to recognize bodily tension in their body, and thereby engage in relaxation. With rewards and praise, a therapist can directly shape the youth's behavior to be more adaptive using positive reinforcement (i.e., providing a desired consequence when the desired behavior is performed) and/or negative reinforcement (i.e., removing or reducing something undesirable such as a chore when the desired behavior is performed) and can also teach the youth's caregiver to administer the rewards. Problem solving is where a youth is taught to identify the problem and brainstorm all possible options on how to solve it. Once this has been accomplished, the next step is to evaluate all positive and negative consequences for each option as well as the advantages and disadvantages, and try out an option. Primary control and secondary control coping are two complementary ways of handling stressful situations. Primary control coping involves actions taken to change the situation and secondary control coping involves actions taken to change the way an individual views the situation. Activity selection helps youth to learn to increase age-appropriate pleasant activities by identifying pleasant activities and arranging their schedule to increase their frequency. Social skills (e.g., learning to start a conversation, listening actively) help youth who are having difficulty making and retaining friendships. Time out and active ignoring are two treatment elements that are commonly used in children with externalizing symptoms. When an undesirable behavior occurs, time out removes the youth from a desirable activity and the youth must be placed in a location that is free from reinforcement, in order to reduce the behavior. Active ignoring teaches caregivers to ignore unwanted behavior and only pay attention to wanted behavior. One on one time and communication skills training are used to improve the relationship between the youth and 
his/her caregiver(s) by increasing time spent together in pleasurable activities, as well as allowing youth and his/her caregiver(s) to practice different ways of talking with each other about challenging situations.

Order of elements from the treatment manuals did not follow the manuals but instead was determined during supervision based on input from the therapist and the supervisor, following the partnership model of shared responsibility for study procedures. Earlier treatment choices were made by both the therapist and supervisor based on a participant's case conceptualization. Decisions were then made during supervision based on the participant's progression through treatment and how well the participant was responding to certain treatment elements. Treatment shifted in the case of a change in the therapist's case conceptualization of the participant or if a new issue arose from previous therapy sessions. In addition to elements from the three manuals, other procedures were introduced and used based on input from the therapists and drawing on the supervisor's knowledge of the evidence base (e.g., communication skills training). In all cases, elements added to a treatment plan were drawn from treatment programs with empirical support. All treatment plans were documented and are described below.

Participant 1 was lost to follow-up so no treatment plan will be reported.

Participant 2's treatment focused on anxiety symptoms and consisted of (a) psychoeducation for child and parent, (b) emotion education, (c) relaxation, (d) fear ladder, (e) cognitive, (f) problem solving, and (g) exposure.

Participant 3's treatment focused on both depression and conduct symptoms and consisted of (a) psycho-education for both the parent and child on depression, (b) activity selection, (c) one on one time, (d) social skills, (e) rewards, (f) psycho-education on conduct 
and ADHD for both child and parent, (g) cognitive, (h) three step plan (i.e., sequential coping from PASCET), and (i) maintenance.

Participant 4's treatment focused on anxiety symptoms and consisted of (a) psychoeducation for child and parent, (b) monitoring of anxiety symptoms, (c) hyperventilation, (d) breathing retraining, (e) cognitive, (f) relaxation, (g) interoceptive exposure, (h) fear ladder, and (i) exposure.

Participant 5's treatment focused on depression symptoms and consisted of (a) psycho-education for child and parent, (b) activity selection, (c) cognitive, and (d) problem solving.

Participant 6's treatment focused on both depression and conduct symptoms and consisted of (a) psycho-education for child and parent on ADHD, (b) praise, (c) one on one time, (d) active ignoring, (e) cognitive, (f) problem solving, (g) rewards, (h) time out, and (i) antecedent management/stimulus control.

Participant 7's treatment focused on anxiety symptoms. Participant 7 did not get very far in treatment and only had two intake sessions with the therapist before stopping therapy.

Participant 8's treatment focused on depression symptoms and consisted of (a) psycho-education for child and parent, (b) activity selection, (c) relaxation, and (d) communication skills training.

\section{Therapists}

Therapists were two full-time masters level clinicians employed by the clinic. Therapist A was a female therapist at the clinic with a Master's degree in social work. She treated participants 3, 6, and, 7. Therapist B was a male therapist at the clinic with Master's degree in clinical psychology. He treated participants 2, 4, 5, and 8. Therapists were trained 
and supervised by Michael Southam-Gerow, Ph.D., study PI. Training took place over a 2day period at Virginia Commonwealth University. Therapists completed a consent procedure. Supervision consisted group meetings and included weekly tape review.

\section{Analytic Plan}

The study will use two methods of analyses: (a) Visual Analyses and (b) Clinical Significance Analyses. Each case will involve analyses for the measures relevant to the presenting symptoms, similar to what Kazdin refers to as "response-specific" measures because the selection of measures to be analyzed for each participant is matched to their particular target problems (1982). Visual analyses will involve graphing participants' scores on the multiple measures for child and parent reports, examining changes in mean and changes in trend across assessment phases for improvement in symptoms, and determining whether any changes are related to treatment. Clinical significance analyses (Kendall, Flannery-Schroeder, \& Ford, 1999) will be employed for measures possessing normative ranges (i.e. K-SADS, CBCL, and RCADS). Participants will be classified as treatment success/failure based on their status on these measures, as compared to normative ranges, at the follow-up assessment points.

\section{Results}

\section{Overview}

In the following section, each participant's data will be presented in graph form for all completed baseline, treatment, and follow-up assessments. For each participant I will first report outcomes for the symptoms and diagnosis domain followed by functioning domain outcomes. The completed battery evolved over time and thus each participant had a somewhat different set of measures. Further, given the focus of treatment differed by 
participant, outcomes for each participant were judged using measures that best indexed the participant's primary focus of treatment. Table 3 summarizes the measurement data that will be presented for each participant.

Table 3.

Treatment Outcome Measures for Each Participant.

\begin{tabular}{|c|c|c|c|}
\hline \begin{tabular}{|c|} 
Participant \\
Number
\end{tabular} & \begin{tabular}{|c|} 
Primary Focus \\
of Treatment
\end{tabular} & $\begin{array}{c}\text { Symptoms and Diagnosis Outcome } \\
\text { Measures }\end{array}$ & $\begin{array}{c}\text { Functioning Outcome } \\
\text { Measures }\end{array}$ \\
\hline 1 & \begin{tabular}{|l|} 
Depression and \\
Externalizing \\
Symptoms
\end{tabular} & $\begin{array}{l}\text { Lost to follow-up. No treatment outcomes } \\
\text { will be reported. }\end{array}$ & $\begin{array}{l}\text { Lost to follow-up. No } \\
\text { treatment outcomes } \\
\text { will be reported. }\end{array}$ \\
\hline 2 & Anxiety & $\begin{array}{l}\text { 1. RCADS Total Anxiety } T \text {-score; } 2 \text {. } \\
\text { CBCL Internalizing Problems and Anxiety } \\
\text { Symptoms } T \text {-scores; } 3 \text {. K-SADS-PL } \\
\text { diagnosis }\end{array}$ & 1. CIS Total Score \\
\hline 3 & \begin{tabular}{|l} 
Depression and \\
Externalizing \\
Symptoms
\end{tabular} & $\begin{array}{l}\text { 1. RCADS Total Depression } T \text {-score; } 2 \text {. } \\
\text { CBCL Internalizing Problems and } \\
\text { Externalizing Problems } T \text {-scores; } 3 \text {. K- } \\
\text { SADS-PL diagnosis }\end{array}$ & 1. CIS Total Score \\
\hline 4 & Anxiety & $\begin{array}{l}\text { 1. RCADS Total Anxiety } T \text {-score; } 2 \text {. } \\
\text { CBCL Internalizing Problems and Anxiety } \\
\text { Symptoms } T \text {-scores; } 3 \text {. SDQ Overall } \\
\text { Stress total scale score; } 4 \text {. K-SADS-PL } \\
\text { diagnosis }\end{array}$ & 1. CIS Total Score \\
\hline 5 & Depression & $\begin{array}{l}\text { 1. RCADS Total Depression } T \text {-score; } 2 \text {. } \\
\text { SDQ Overall Stress total scale score; } 3 . \mathrm{K} \text { - } \\
\text { SADS-PL diagnosis }\end{array}$ & 1. BIS Total Score \\
\hline 6 & $\begin{array}{l}\text { Depression and } \\
\text { Externalizing } \\
\text { Symptoms }\end{array}$ & $\begin{array}{l}\text { 1. RCADS Total Depression } T \text {-score; } 2 \text {. } \\
\text { SDQ Overall Stress total scale score; } 3 . \mathrm{K} \text { - } \\
\text { SADS-PL diagnosis }\end{array}$ & 1. BIS Total Score \\
\hline 7 & Anxiety & $\begin{array}{l}\text { 1. RCADS Total Anxiety } T \text {-score; 2. SDQ } \\
\text { Overall Stress total scale score; 3. K- } \\
\text { SADS-PL diagnosis }\end{array}$ & 1. BIS Total Score \\
\hline 8 & Depression & $\begin{array}{l}\text { 1. RCADS Total Depression } T \text {-score; } 2 \text {. } \\
\text { SDQ Overall Stress total scale score; } 3 . \mathrm{K} \text { - } \\
\text { SADS-PL diagnosis }\end{array}$ & 1. BIS Total Score \\
\hline
\end{tabular}


$T$-scores above 65 on the Revised Child Anxiety and Depression Scale (RCADS) are considered indicative of "clinically significant" symptoms (Chorpita et al., 2000). T-scores above 60 on the Child Behavior Checklist (CBCL) Internalizing and Externalizing Problems scale are considered to indicate clinical significance, whereas $T$-scores above 65 on the Child Behavior Checklist (CBCL) Anxiety Syndrome scale are considered to indicate clinical significance (Achenbach \& Rescorla, 2001). The RCI of 4.8 points will also be used to indicate clinically significant change on the CBCL Internalizing and Externalizing Problems scale (McClendon et al., 2011). Overall Stress scale scores on the Symptoms and Difficulties Questionnaire (SDQ) from 14 to 16 are considered "borderline" while those scores above 17 are considered to indicate "abnormal" levels of overall stress (Goodman, 2001). For the functioning domain, a score of 16 or greater on the CIS is considered indicative of definite impairment, while a score of 14 or greater on the BIS is considered indicative of definite impairment (Bird et al., 1993).

Both the symptoms and diagnosis outcome measures and the functioning outcome measures graphs will contain constant elements to assist in visual and clinical significance analyses. For measures with a clinical cutoff score (i.e., RCADS, CBCL, SDQ), the clinical cutoff will be graphed for each assessment point in order to visually indicate when a participant's scores have fallen below or above this line. For all measures, the mean phase scores will be graphed in order to indicate a change in mean level for each assessment phase. The graphs will also contain a linear trend line in order to indicate whether a change in trend has occurred throughout the assessment phases.

\section{Participant 1}


As stated previously, Participant 1 was lost to follow-up. Due to a lack of data for comparison across phases, this participant was excluded from analysis.

\section{Participant 2}

\section{Symptoms and diagnosis domain.}

Child report measure. Participant 2 completed eight total assessments ( 3 baseline, 3 treatment, and 2 follow-up). The results of Participant 2's self-reported levels of anxiety using the Total Anxiety scale of the Revised Child Anxiety and Depression Scale (RCADS) are presented in Figure 1. These results indicate that Participant 2 experienced his highest level of anxiety $(T$-score $=50)$ at the first baseline assessment. Participant 2's Total Anxiety $T$-scores mean for each assessment phase declined over baseline (mean $T$-score $=45)$, treatment $($ mean $T$-score $=38.3)$, and follow-up $($ mean $T$-score $=32.6)$. The results also indicate a linear trend towards decreasing anxiety symptoms from baseline to follow-up.

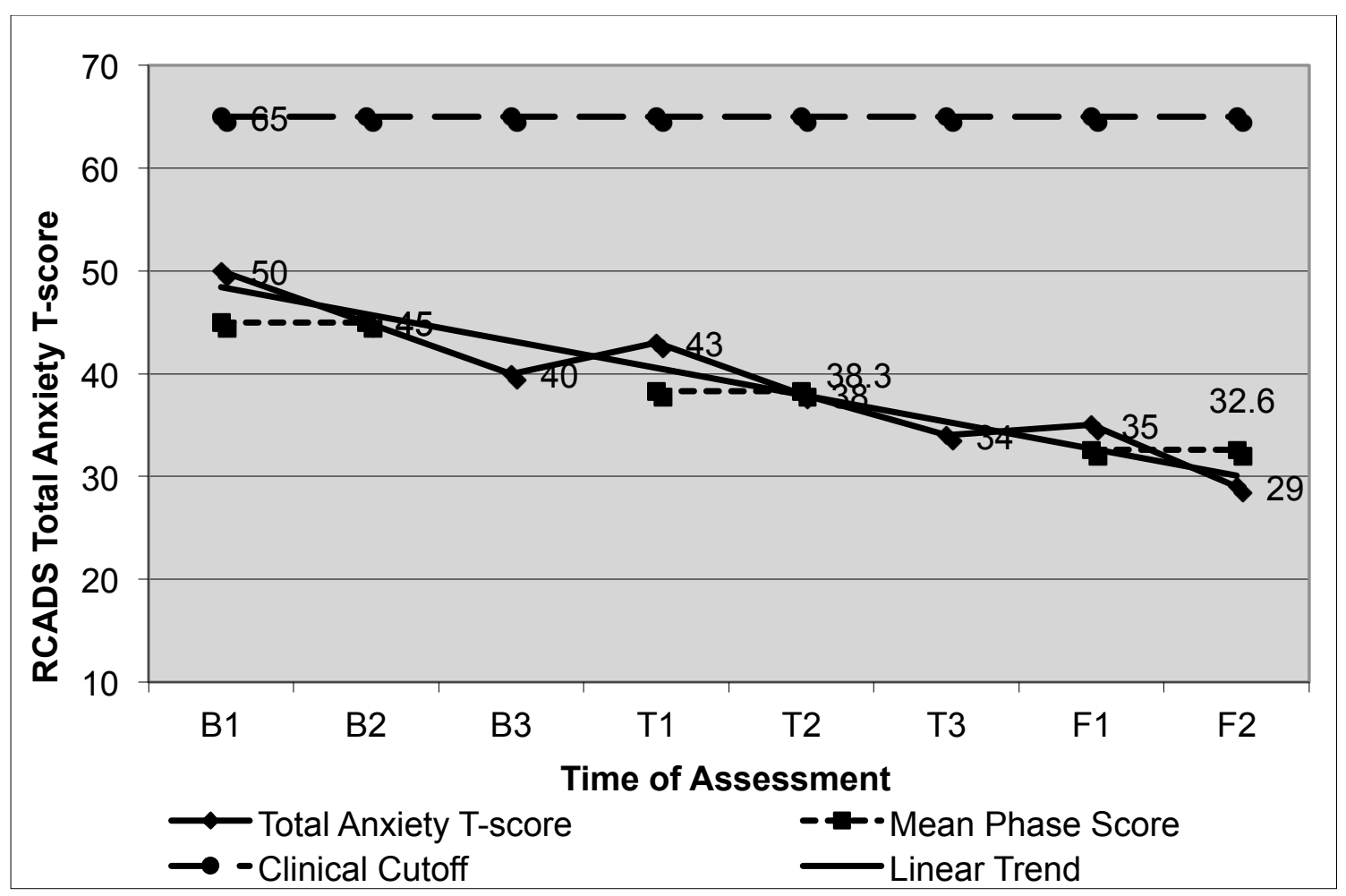

Figure 1. RCADS T-scores across baseline, treatment, and follow-up for participant 2. 
While Participant 2's results show a general reduction in Total Anxiety $T$-scores from baseline to treatment and treatment to follow-up, he never demonstrated $T$-scores in the clinically significant range.

Parent report measures. The results of Participant 2's parent report of internalizing behavior problems on the Child Behavior Checklist (CBCL) Internalizing Problems scale are displayed in Figure 2. Parental report reveals a linear trend of consistent decline in $T$-scores throughout the assessment phases. Though results indicate a relatively stable but declining trend from the first baseline to the first treatment assessment, these reports indicate an increase in internalizing behaviors at the second treatment assessment $(T$-score $=63)$.

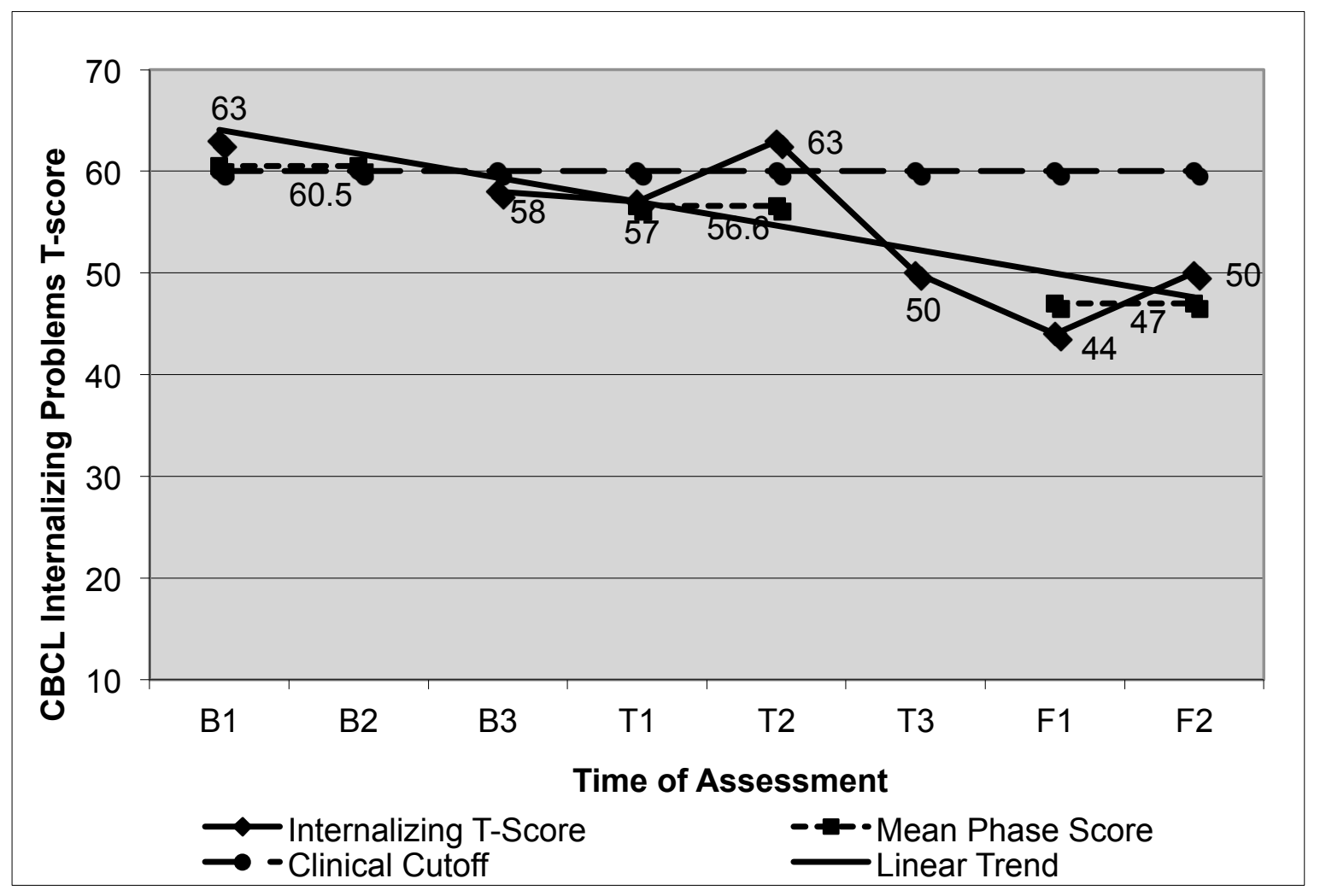

Figure 2. CBCL Internalizing T-scores across baseline, treatment, and follow-up for participant 2. 
Participant 2's Internalizing Problems $T$-scores mean for each assessment phase declined over baseline $($ mean $T$-score $=60.5)$, treatment $($ mean $T$-score $=56.6)$, and follow-up $($ mean $T$-score $=47)$. These results reveal a visually significant decrease in parental report of internalizing behavior from baseline to follow-up. These results also meet criteria for reliable change with a reduction of 13.5 points from baseline mean score to follow-up mean score. Further, reports of internalizing behaviors in the clinically significant range at baseline were no longer significant by the final follow-up. So, these findings indicate that according to scores on the CBCL Internalizing scale, Participant 2 can be categorized as recovered. The results of Participant 2's parent report of anxiety symptoms on the Child Behavior Checklist (CBCL) Anxiety syndrome scale are displayed in Figure 3. Parental report reveals a linear trend of a decrease in $T$-scores throughout the assessment phases. Though results indicate a relatively stable but declining trend from the first baseline to the first treatment assessment, these reports indicate an increase in anxiety at the second treatment assessment $(T$-score $=70)$. Participant 2's Anxiety syndrome scale $T$-scores mean for each assessment phase declined over baseline (mean $T$-score $=71.5$ ), treatment (mean $T$ score $=66)$, and follow-up (mean $T$-score $=58)$. 


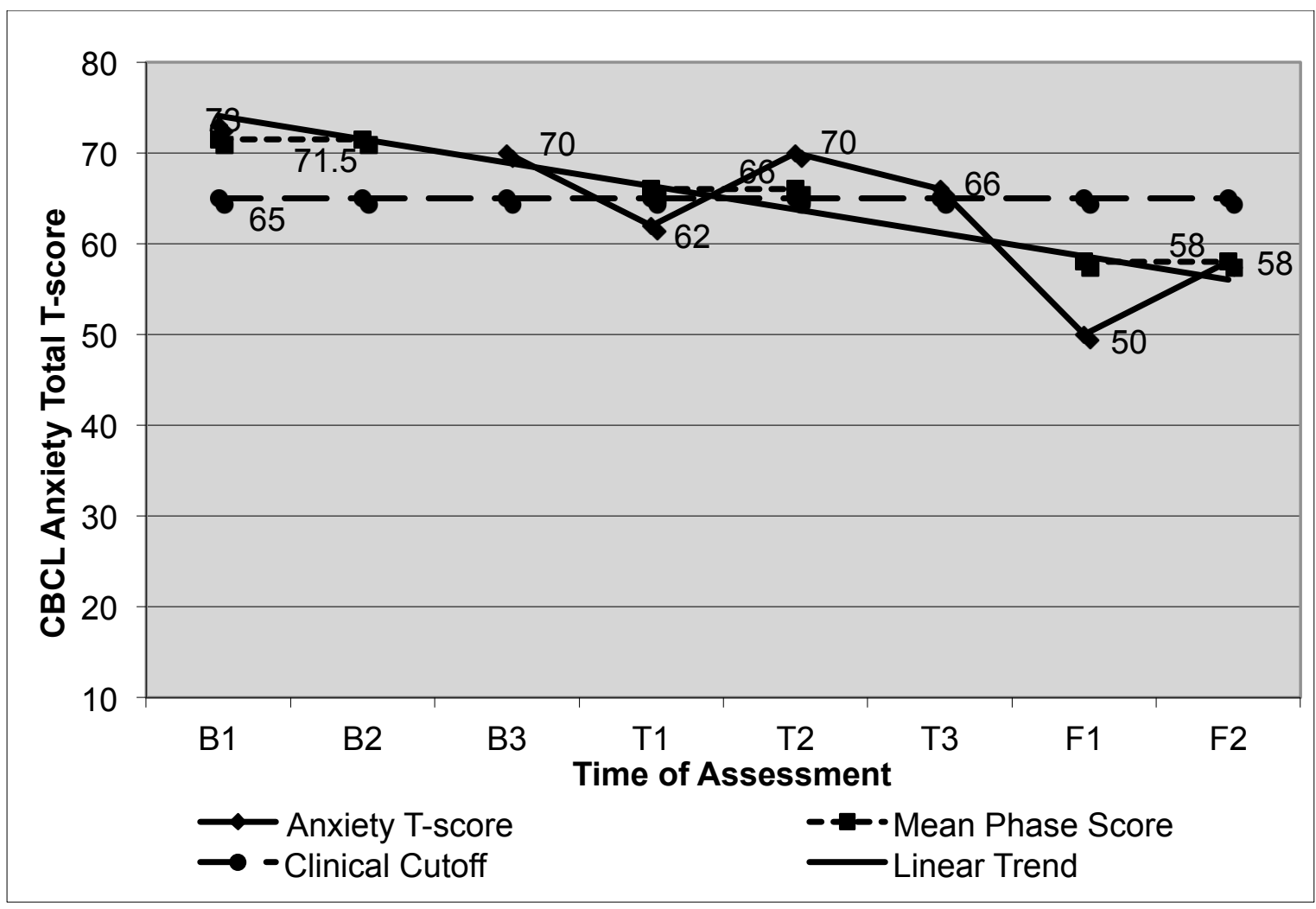

Figure 3. CBCL Anxiety T-scores across baseline, treatment, and follow-up for participant 2.

These results reveal a visually significant decrease in parental report of anxiety from baseline to follow-up. Further, reports of anxiety in the clinically significant range at baseline were no longer significant by the final follow-up.

The initial diagnostic interview using the parent report version of the K-SADS-PL indicated that Participant 2's behavior reached criteria for Social Phobia. This was the only disorder for which his behaviors reached diagnostic criteria at the initial baseline assessment. Results indicate that Participant 2 did not reach diagnostic criteria for Social Phobia or for any other DSM-IV disorder at any point during the follow-up assessments.

\section{Functioning Domain.}

Parent report measure. The results of Participant 2's parent report of functional impairment on the Columbia Impairment Scale (CIS) and represented as total scale scores are 
displayed in Figure 4. Results indicate a declining trend from the first baseline assessment to the last follow-up assessment. Participant 2's CIS total scale scores mean for each assessment phase declined over baseline (mean scale score $=11.6)$, treatment $($ mean scale score $=8)$, and follow-up (mean scale score $=2.5$ ). Further, these results reveal a visually significant decrease in parental report of functional impairment from baseline to follow-up.

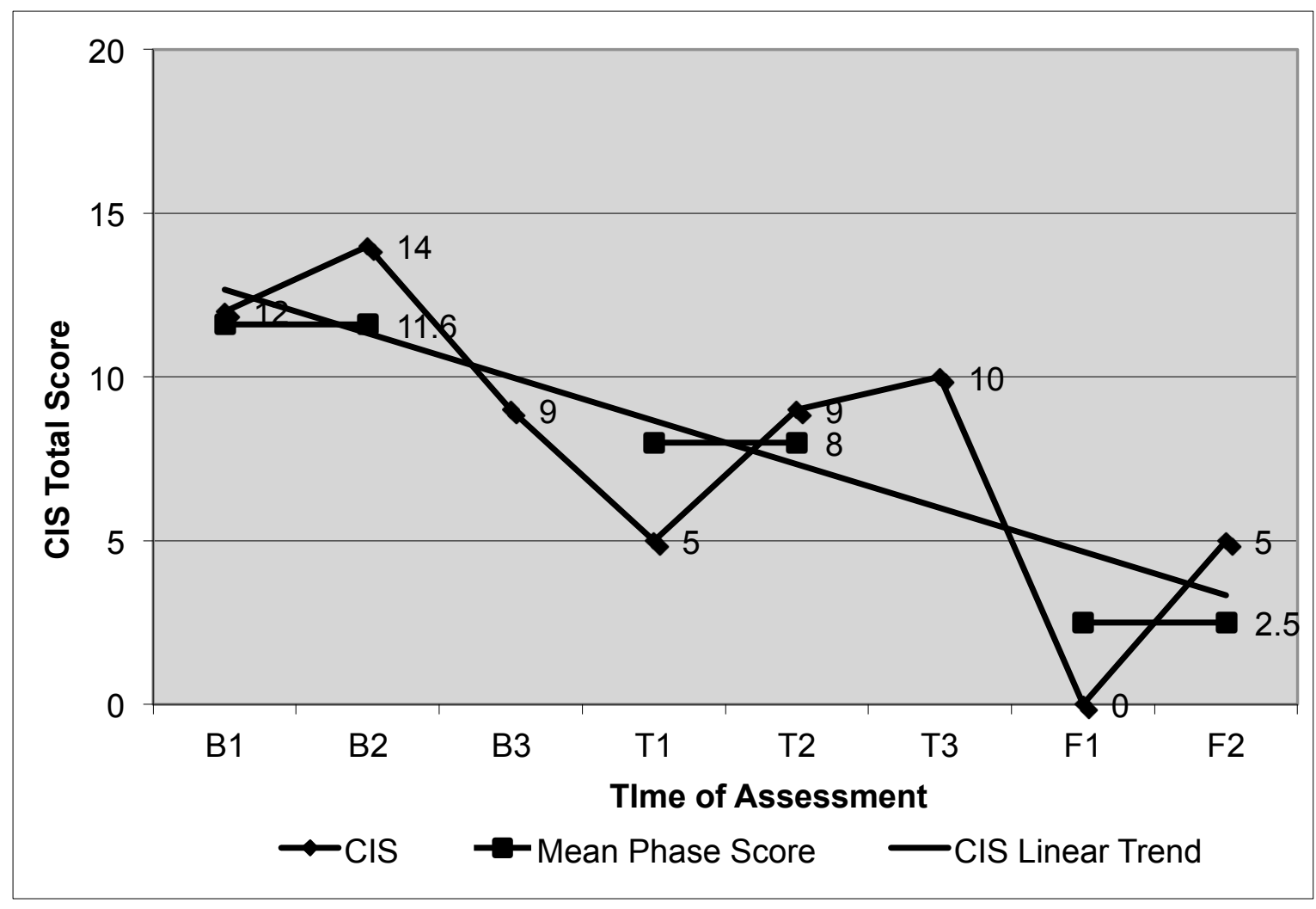

Figure 4. CIS total scale scores across baseline, treatment, and follow-up for participant 2.

Overall, Participant 2 is a treatment success. For child-reported symptoms for the RCADS, although participant 2 was never "clinically significant" his anxiety symptoms decreased from baseline to follow-up. Both parent-reported CBCL scales that were "clinically significant" at baseline were no longer "clinically significant" at follow-up, and the CBCL Internalizing broadband scale decrease of 13.5 points from baseline to follow-up 
indicated reliable change based on the RCI. This indicates that Participant 2 can be classified as recovered, both passing clinical cutoff and meeting RCI criteria.

Results also demonstrate that Participant 2 no longer met diagnostic criteria for any DSM-IV disorder at follow-up. Parent-reported functioning also indicated a decline in functional impairment from baseline to follow-up.

\section{Participant 3}

\section{Symptoms and diagnosis domain.}

Child report measure. Participant 3 completed twelve total assessments ( 5 baseline, 5 treatment, and 2 follow-up). The results of Participant 3's self-reported levels of depression using the Total Depression scale of the Revised Child Anxiety and Depression Scale (RCADS) and represented as overall $T$-scores are presented in Figure 5. These results indicate that Participant 3 experienced his highest level of depression $(T$-score $=50)$ at the first baseline assessment, and that his depression during treatment and follow-up phases remained consistently between $T$-scores of 36 and 55 . 


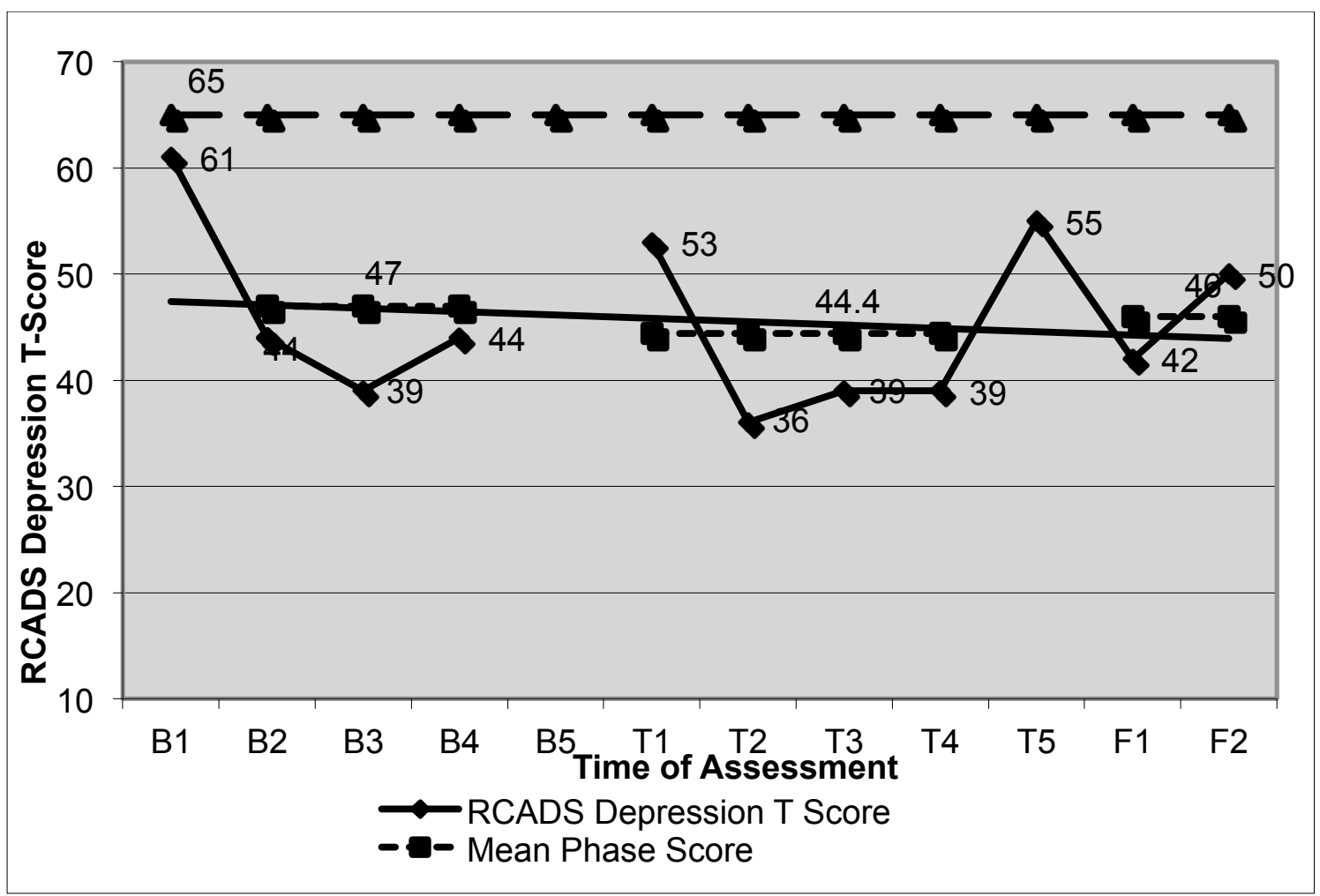

Figure 5. RCADS T-scores across baseline, treatment, and follow-up for participant 3.

Participant 3's Total Depression $T$-scores declined over baseline and remained relatively stable over treatment and follow-up. Participant 3's Total Depression T-scores mean for each assessment phase also remained relatively stable during baseline (mean $T$-score $=47$ ), treatment $($ mean $T$-score $=44.4)$, and follow-up $($ mean $T$-score $=46)$. The results also indicate a minimal downward linear trend in depression symptoms from baseline to followup. RCADS $T$-scores above 65 are considered indicative of "clinically significant" symptoms (Chorpita et al., 2000). While Participant 3 showed minimal mean change in Total Depression, he never demonstrated $T$-scores in the clinically significant range. 




Figure 6. CBCL Total Internalizing T-scores across baseline, treatment, and follow-up for participant 3 .

Parent report measures. The results of Participant 3's parent report of internalizing behavior problems on the Child Behavior Checklist (CBCL) Internalizing Problems scale and represented as $T$-scores are displayed in Figure 6. Parental report reveals a relatively stable but slight decline of linear trend in $T$-scores throughout the assessment phases. Though these results reveal minimal change in $T$-score means from baseline (mean $T$-score $=74)$ to treatment $($ mean $T$-score $=73.5)$, these results indicate a decrease in the mean Internalizing $T$-score at follow-up (mean $T$-score $=68) . T$-scores above 60 on the CBCL Internalizing Problems scale are considered to indicate clinical significance (Achenbach \& Rescorla, 2001). Although these results reveal a slight decline in parental report of internalizing behavior from baseline to follow-up and meet criteria for reliable change with a decrease of 6 
points from baseline to follow-up, parental report indicates that Participant 3's internalizing behavior problems are still clinically significant. This suggests that Participant 3's scores are improved but not recovered, because although he passed RCI criteria, he did not pass the clinical cutoff.



Figure 7. CBCL Externalizing T-scores across baseline, treatment, and follow-up for participant 3.

The results of Participant 3's parent report of externalizing behavior problems on the Child Behavior Checklist (CBCL) Externalizing Problems scale and represented as $T$-scores are displayed in Figure 7. Parental report indicates that Participant 3 experienced his highest level of externalizing behavior at the first baseline assessment $(T$-score $=82)$. These results reveal a relatively stable decline in linear trend of $T$-scores throughout the assessment phases. Parental report indicates a decrease in $T$-score means from baseline (mean $T$-score $=79)$, 
treatment $($ mean $T$-score $=71.75)$, and follow-up $($ mean $T$-score $=72) . T$-scores above 60 on the CBCL Externalizing Problems scale are considered to indicate clinical significance (Achenbach \& Rescorla, 2001). Although these results reveal a visually significant decrease in parental report of externalizing behavior from baseline to follow-up and meet criteria for reliable change with a decrease of 8 points from baseline to follow-up, parental report indicates that Participant 3's externalizing behavior problems are still in the clinically significant range. This suggests that although Participant 3 met RCI criteria, he can only be categorized as improved because his scores do not fall below the clinical cutoff point.

The initial diagnostic interview using the parent report version of the K-SADS-PL indicated that Participant 3's behavior reached criteria for Minor Depression, ADHD, and ODD. These were the only disorders for which his behaviors reached diagnostic criteria at the initial baseline assessment. Results indicate that Participant 3 did not meet criteria for Minor Depression and ODD at both follow-up assessments. Participant 3 however, still met diagnostic criteria for ADHD at both follow-up assessments.

\section{Functioning Domain.}

Parent report measure. The results of Participant 3's parent report of functional impairment on the Columbia Impairment Scale (CIS) and represented as total scale scores are displayed in Figure 8. The results demonstrate that Participant 3's CIS total scale scores remained relatively stable during the baseline phase (scale scores ranging from 40 to 35) with a mean scale score of 38.4 , and then decreased with slight variability across treatment (mean scale score $=28.5$ ). During the follow-up phase, Participant 3's scale scores increased to a mean scale score of 35.5. Further, these results reveal a slight linear decrease in parental report of functional impairment from baseline to follow-up. 




Figure 8. CIS total scale scores across baseline, treatment, and follow-up for participant 3.

Overall, Participant 3 has poor outcomes, suggesting treatment was not successful. For child-reported symptoms for the RCADS, although participant 3 was never "clinically significant" his anxiety symptoms only decreased slightly from baseline to follow-up. Both parent-reported CBCL scales that were "clinically significant" at baseline remained "clinically significant" at follow-up. Results also demonstrate that Participant 3 still met criteria for ADHD at follow-up, but did not meet criteria for his other two baseline diagnoses (minor depression, ODD). Parent-reported functioning only indicated a minimal decline in functional impairment from baseline to follow-up. Participant 3's mean score on the BIS at follow-up (35.5) indicated definite impairment.

\section{Participant 4}

\section{Symptoms and diagnosis domain.}


Child report measure. Participant 4 completed eight total assessments ( 3 baseline, 2 treatment, and 3 follow-up). The results of Participant 4's self-reported levels of anxiety using the Total Anxiety scale of the Revised Child Anxiety and Depression Scale (RCADS) and represented as overall $T$-scores are presented in Figure 9. These results indicate that Participant 4 experienced his highest level of anxiety $(T$-score $=66)$ at the first baseline assessment. Participant 4's Total Anxiety T-scores mean for each assessment phase declined over baseline $($ mean $T$-score $=63.3)$, treatment $($ mean $T$-score $=55)$, and follow-up $($ mean $T$ score $=33.3$.

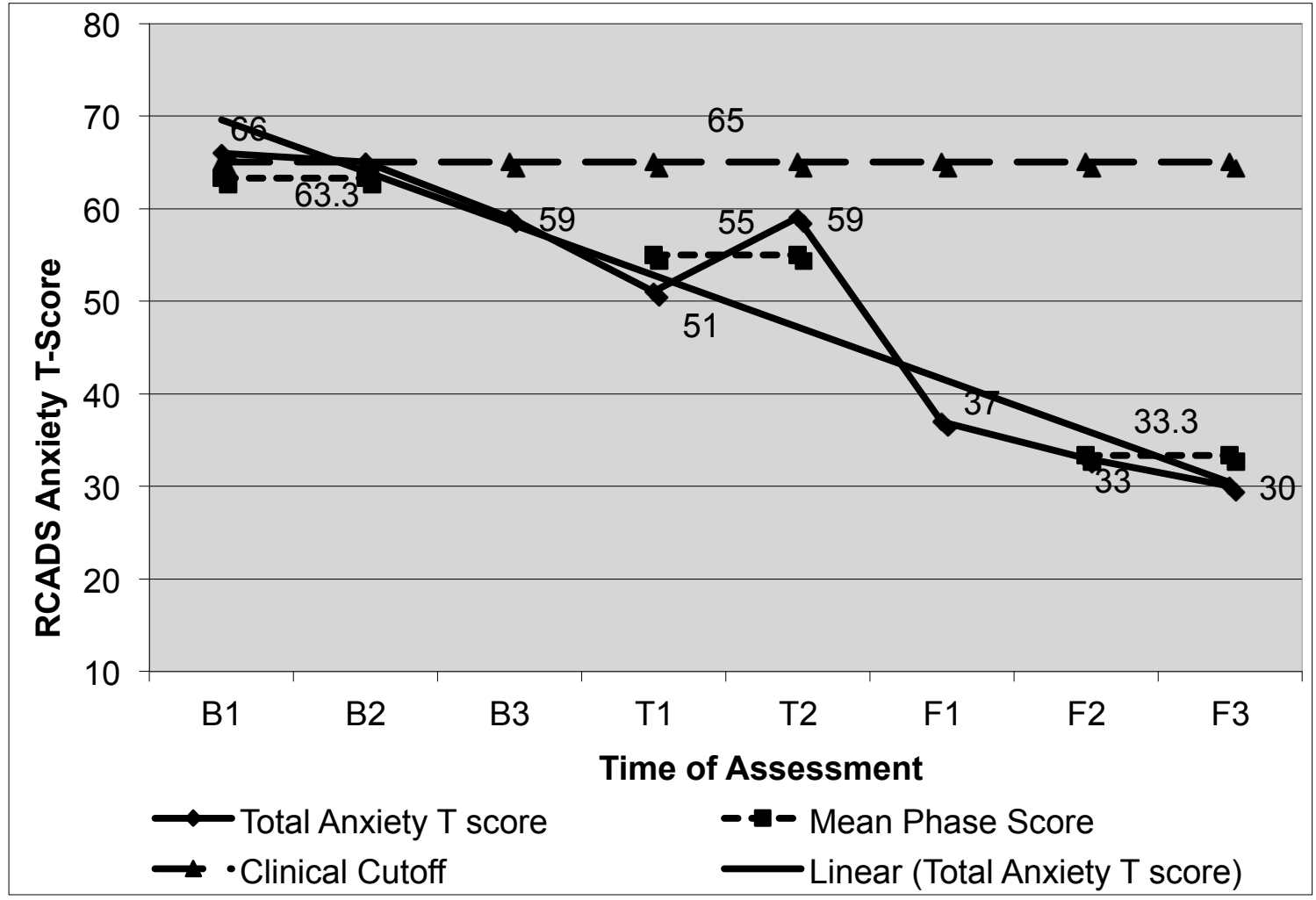

Figure 9. RCADS Total Anxiety T-Scores across baseline, treatment, and follow-up for participant 4.

The results also indicate a visually significant linear trend of decreasing anxiety symptoms from the first baseline assessment to the last follow-up. RCADS $T$-scores above 65 are 
considered indicative of "clinically significant" symptoms (Chorpita et al., 2000). Participant 4's Anxiety $T$-scores were within the range of clinical significance in the first $(T$-score $=66)$ and second baseline $(T$-score $=65)$ assessment and were below the level of clinical significance for the last baseline assessment. Participant 4's Anxiety $T$-scores remained below the clinical range throughout treatment and follow-up assessments.



Figure 10. CBCL Internalizing T-scores across baseline, treatment, and follow-up for participant 4.

Parent report measures. The results of Participant 4's parent report of internalizing behavior problems on the Child Behavior Checklist (CBCL) Internalizing Problems scale and represented as $T$-scores are displayed in Figure 10. The results demonstrate that parental report of Participant 4's internalizing behavior problems remained relatively stable during the baseline phase ( $T$-scores ranging from 65 to 62 ), and then decreased with slight variability 
across treatment and follow-up. The results of the CBCL Internalizing $T$-scores graph indicates that Participant 4 received scores in the range of clinical significance during baseline, but by the first treatment assessment his scores had fallen below a clinical level ( $T$ score $=57$ ). Participant 4's Internalizing Problems $T$-scores mean for each assessment phase declined over baseline $($ mean $T$-score $=63.5)$, treatment $($ mean $T$-score $=54.5)$, and followup (mean $T$-score $=44.6)$, indicating reliable change with a decrease of 18.9 points from baseline to follow-up. This indicates that Participant 4 can be categorized as recovered due to both meeting RCI criteria and no longer being above the clinical cutoff. $T$-scores above 60 on the CBCL Internalizing Problems scale are considered to indicate clinical significance (Achenbach \& Rescorla, 2001). These results reveal a visually significant linear decrease in parental report of internalizing behavior from baseline to follow-up. Further, reports of internalizing behaviors in the clinically significant range at baseline were no longer significant by the final follow-up. 


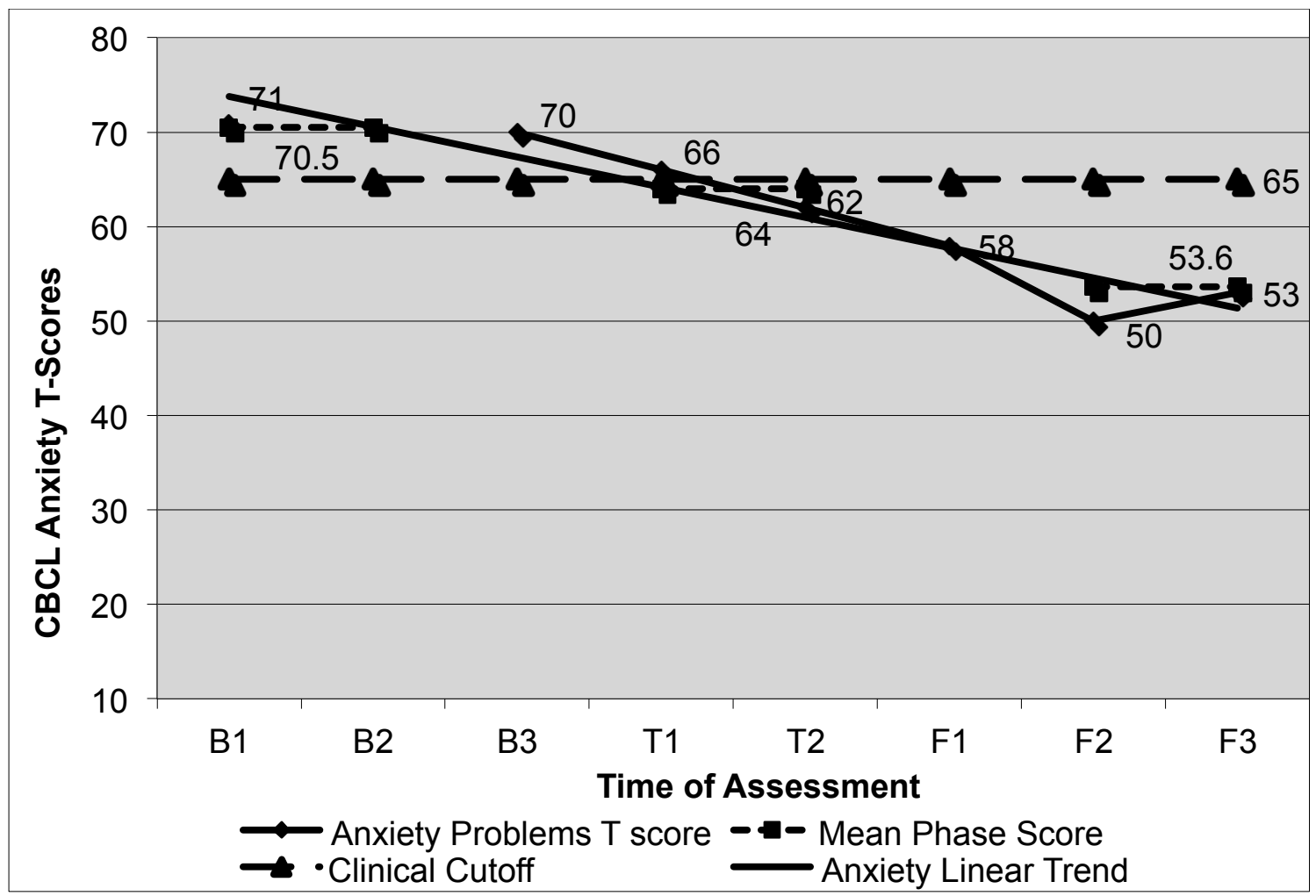

Figure 11. CBCL Anxiety T-scores across baseline, treatment, and follow-up for participant 4.

The results of Participant 4's parent report of anxiety symptoms on the Child Behavior Checklist (CBCL) Anxiety syndrome scale and represented as $T$-scores are displayed in Figure 11. The results demonstrate that parental report of Participant 4's anxiety problems remained relatively stable during the baseline phase ( $T$-scores ranging from 71 to 70), and then decreased with slight variability across treatment and follow-up. The results of the CBCL Anxiety $T$-scores graph indicates that Participant 4 received scores in the range of clinical significance during baseline, but by the second treatment assessment his scores had fallen below a clinical level $(T$-score $=62)$. Participant 4's Anxiety $T$-scores mean for each assessment phase declined over baseline $($ mean $T$-score $=70.5)$, treatment $($ mean $T$-score $=$ 64), and follow-up (mean $T$-score $=53.6) . T$-scores above 65 on the CBCL Anxiety 
syndrome scale are considered to indicate clinical significance (Achenbach \& Rescorla, 2001). These results reveal a visually significant decrease in parental report of anxiety from baseline to follow-up. Further, reports of anxiety in the clinically significant range at baseline were no longer significant by the final follow-up.

The results of Participant 4's parent report of levels of overall stress using the Symptoms and Difficulties Questionnaire (SDQ) and represented as overall scale scores are presented in Figure 12. The results indicate that Participant 4's SDQ scores decreased from a mean score of 12.3 at baseline down to 8.5 at treatment, and declined to a 6 at the last two follow-up assessments. The results of Participant 4's parental reports of Overall Stress scores graph indicates that he received a score in the borderline range in the second baseline assessment ( score $=15)$, but by the third baseline assessment his scores had fallen into the normal range of scores. These results reveal a visually significant decrease in parental report of overall stress from baseline to follow-up.

The initial diagnostic interview using the parent report version of the K-SADS-PL indicated that Participant 4's behavior reached criteria for Panic Disorder and Separation Anxiety Disorder. These were the only disorders for which his behaviors reached diagnostic criteria at the initial baseline assessment. Results indicate that Participant 4 did not reach diagnostic criteria for Panic Disorder or Separation Anxiety Disorder, or for any other DSMIV disorder at any point during the follow-up assessments. 


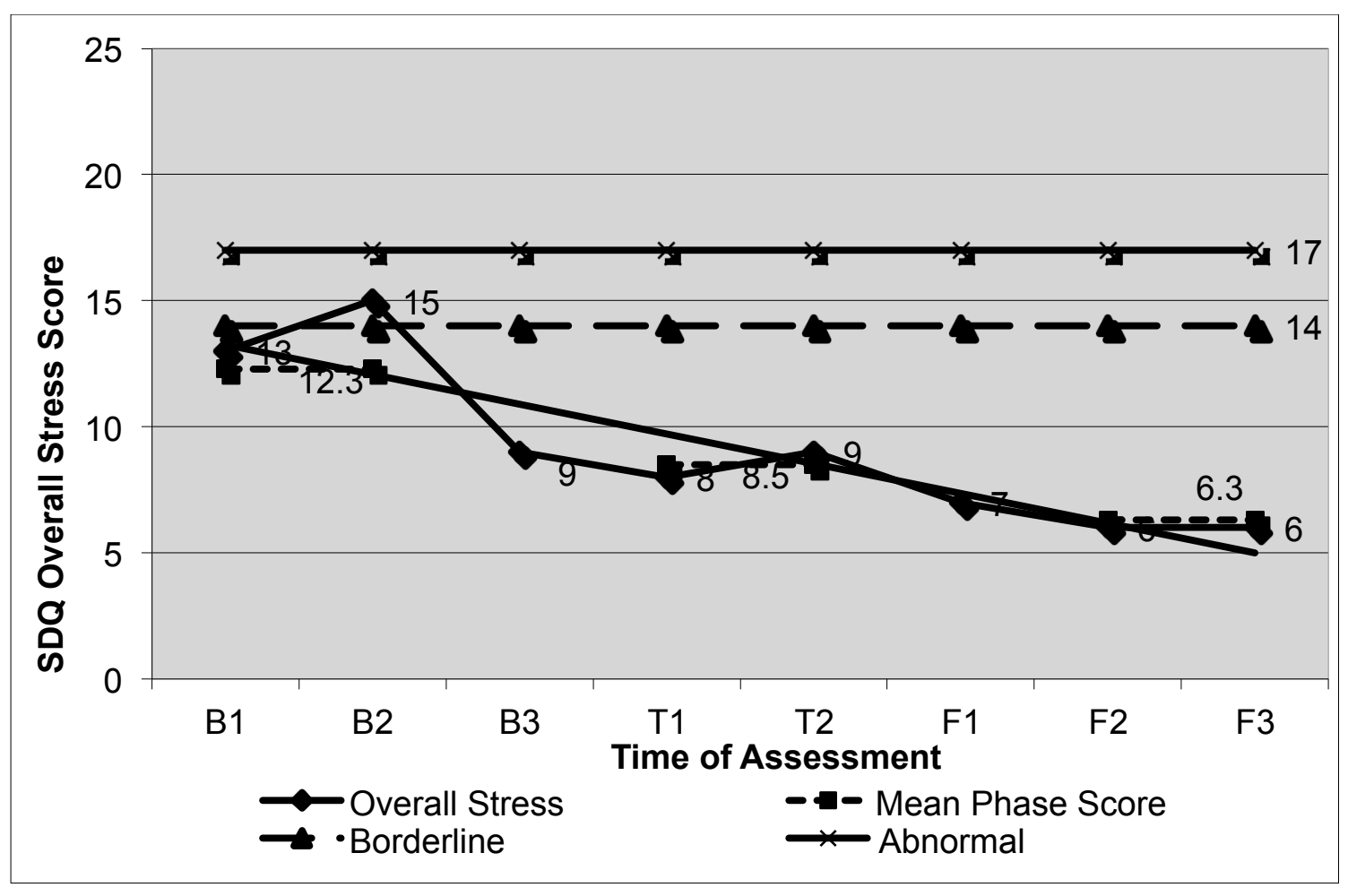

Figure 12. SDQ Overall Stress scores across baseline, treatment, and follow-up for participant 4.

\section{Functioning Domain.}

Parent report measure. The results of Participant 4's parent report of functional impairment on the Columbia Impairment Scale (CIS) and represented as total scale scores are displayed in Figure 13. The results demonstrate that Participant 4's CIS total scale scores remained relatively stable during the baseline phase (scale scores ranging from 18 to 16 ), and then sharply decreased to 9 at the first treatment assessment to a low of 2 at the second follow-up assessment, before increasing slightly to 7 at the final follow-up. Participant 4's CIS total scale scores mean for each assessment phase decreased sharply from baseline $($ mean scale score $=17$ to treatment $($ mean scale score $=6.5)$, and then decreased slightly from treatment to follow-up (mean scale score $=4.3$ ). Further, these results reveal a visually 
significant linear decrease in parental report of functional impairment from baseline to follow-up.

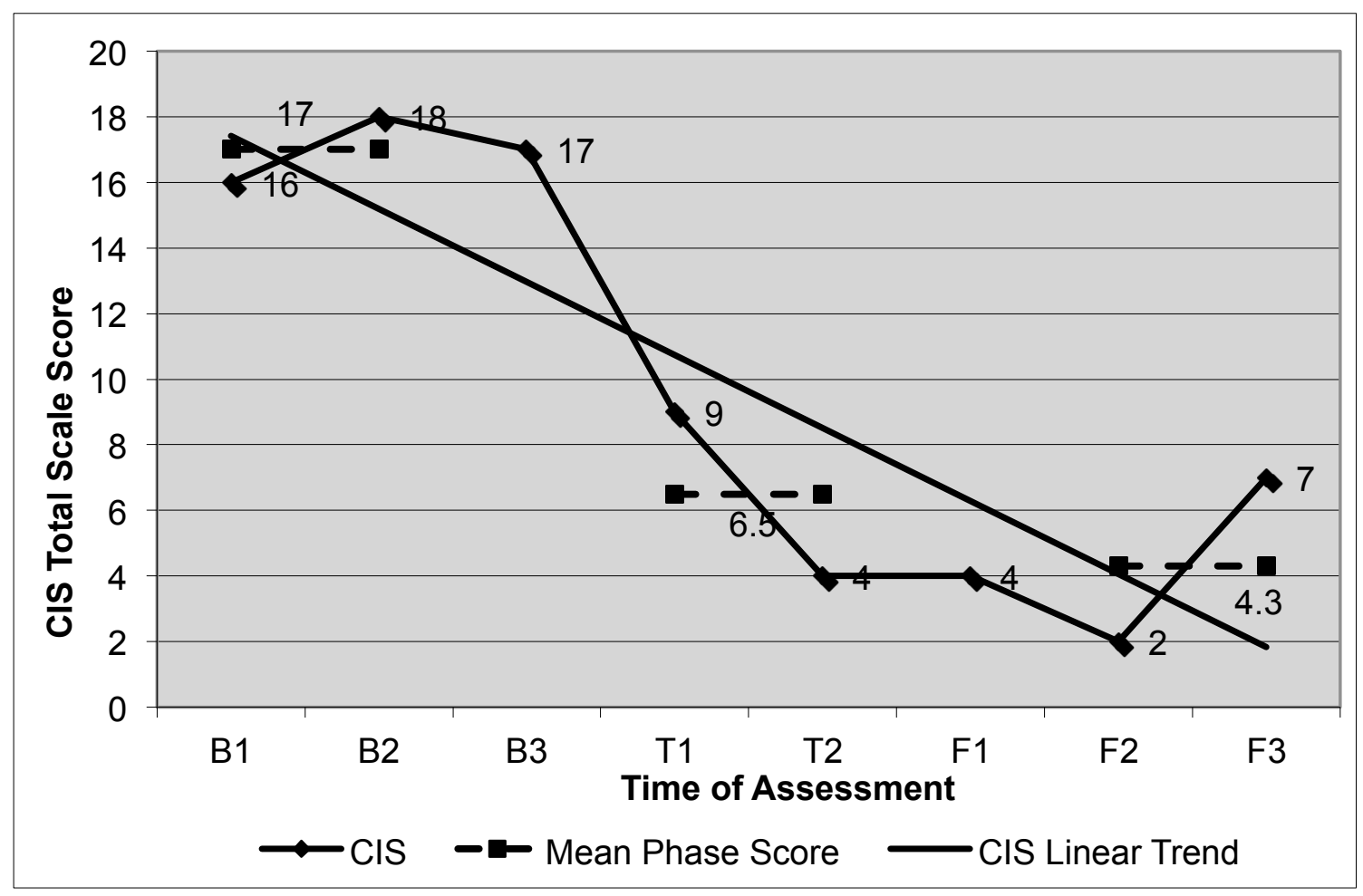

Figure 13. CIS total scale scores across baseline, treatment, and follow-up for participant 4 .

Overall, Participant 4 is a treatment success. For child-reported anxiety symptoms on the RCADS, participant 4 was "clinically significant" at baseline and fell below the clinical cutoff at follow-up. Both parent-reported CBCL scales that were "clinically significant" at baseline were no longer "clinically significant" at follow-up. In addition, the CBCL Internalizing scale score decrease of 18.9 points from baseline to follow-up was indicative of reliable change. This indicates that, according to Participant 4's CBCL scores, he can be categorized as recovered. Parent reported Overall Stress on the SDQ decreased from borderline at baseline to normal at follow-up. Results also demonstrate that Participant 4 no longer met diagnostic criteria for any DSM-IV disorder at follow-up. Parent-reported 
functioning also indicated a decline in functional impairment from baseline to follow-up no longer indicating definite impairment.

\section{Participant 5}

\section{Symptoms and Diagnosis domain.}

Child report measure. Participant 5 completed nine total assessments (4 baseline, 2 treatment, and 3 follow-up). The results of Participant 5's self-reported levels of depression using the Total Depression scale of the Revised Child Anxiety and Depression Scale (RCADS) and represented as overall $T$-scores are presented in Figure 14. These results indicate that Participant 5 experienced his highest level of depression $(T$-score $=65)$ at the fourth baseline assessment. Participant 5's Total Depression $T$-scores mean for each assessment phase declined over baseline (mean $T$-score $=62)$, treatment $($ mean $T$-score $=54)$, and follow-up (mean $T$-score $=47.3)$. The results also indicate a visually significant linear trend of decreasing depression symptoms from the first baseline assessment to the last follow-up. Participant 5's Depression $T$-scores were within the range of clinical significance in the fourth baseline $(T$-score $=65)$. Results indicate Depression $T$-scores remained below the clinical range throughout treatment and follow-up assessments. 


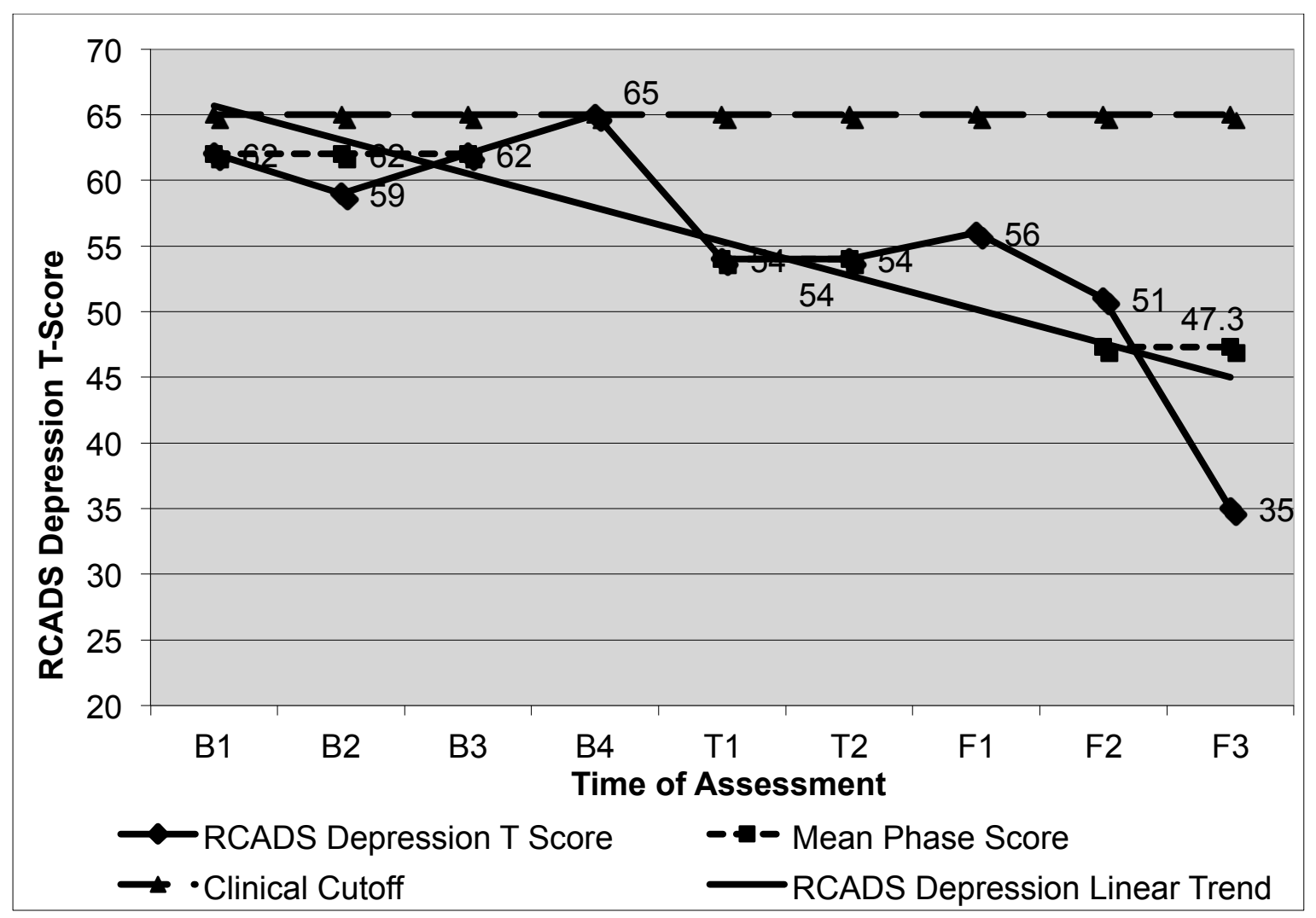

Figure 14. RCADS Depression T-scores across baseline, treatment, and follow-up for participant 5.

Parent report measures. The results of Participant 5's parent report of levels of overall stress using the Symptoms and Difficulties Questionnaire (SDQ) and represented as overall scale scores are presented in Figure 15. The results indicate that Participant 5's SDQ scores decreased from a mean score of 15.5 at baseline down to 14 at treatment, and declined to a 9.6 at the last three follow-up assessments. Participant 5's parental reports of Overall Stress scores graph indicates that her first baseline assessment Overall Stress score is in the borderline range $($ score $=16)$ and her Overall Stress score at the second baseline assessment is in the abnormal range of scores $($ score $=18)$. Results indicate that scores continued to decline the third and fourth baseline assessments as well as the first treatment assessment, before increasing into the abnormal range again $($ score $=18)$. Participant 5's Overall Stress 
scores remained in the normal range of scores throughout follow-up. These results reveal a visually significant decrease in parental report of overall stress from baseline to follow-up.

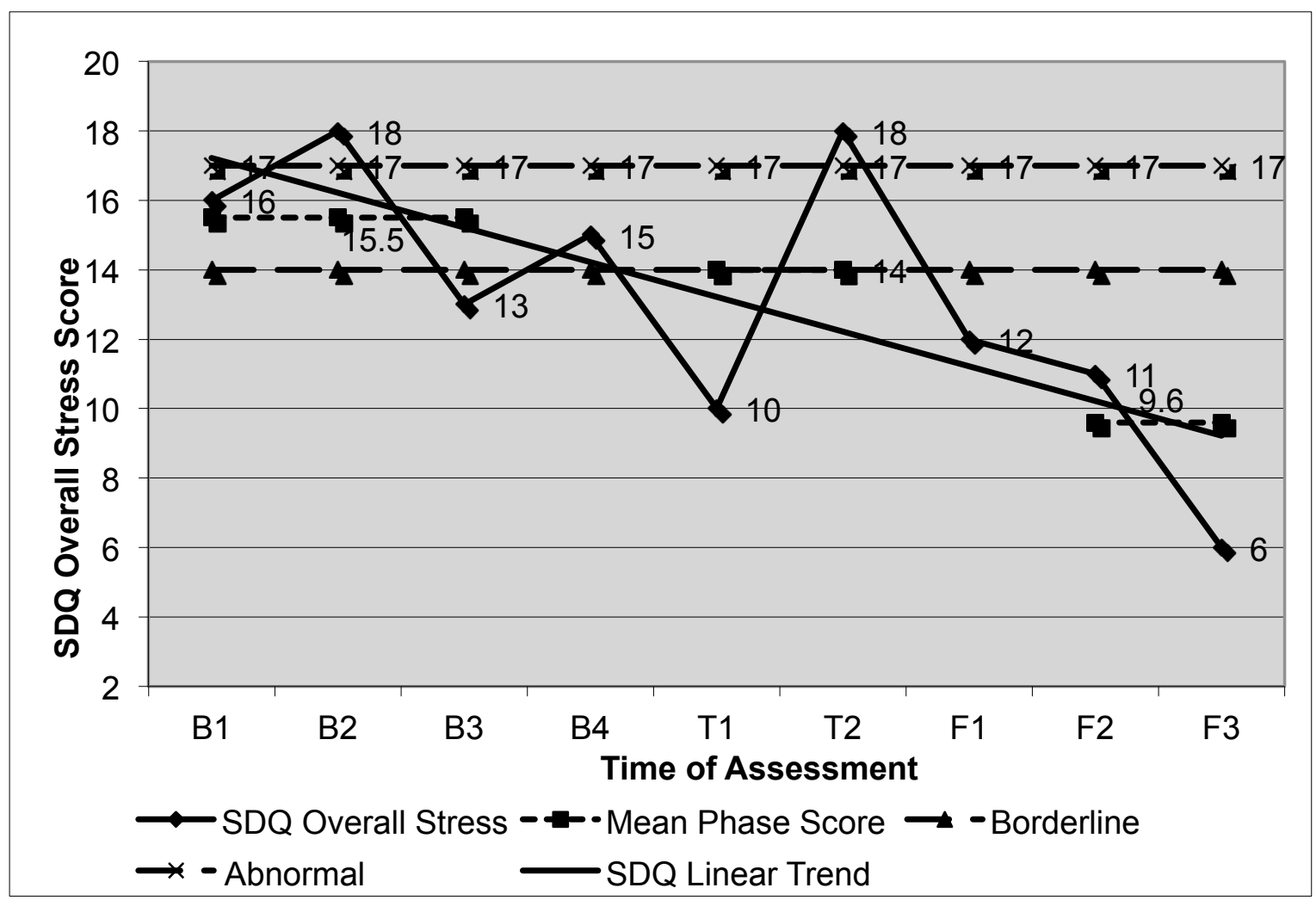

Figure 15. SDQ Overall Stress scores across baseline, treatment, and follow-up for participant 5.

The initial diagnostic interview using the parent report version of the K-SADS-PL indicated that Participant 5's behavior reached criteria for Minor Depression. This was the only disorder for which her behaviors reached diagnostic criteria at the initial baseline assessment. Results indicate that Participant 5 did not reach diagnostic criteria for Major Depression, or for any other DSM-IV disorder at any point during the follow-up assessments.

\section{Functioning Domain.}

Parent report measure. The results of Participant 5's parent report of functional impairment on the Brief Impairment Scale (BIS) and represented as scale scores are 
displayed in Figure 16. The results demonstrate that Participant 5's BIS total scale scores remained relatively stable during the baseline phase (scale scores ranging from 19 to 14) and the treatment phase (18 to 13 ), and then decreased to 9 at the first follow-up assessment before increasing to 12 at the final follow-up. Parental report indicated that the CIS total scale score mean decreased from baseline (mean scale score $=17)$, to treatment (mean scale score $=15.5)$, and to follow-up (mean scale score $=12.3)$. Further, these results reveal a visually significant linear decrease in parental report of functional impairment from baseline to follow-up.



Figure 16. BIS total scale scores across baseline, treatment, and follow-up for participant 5.

Overall, Participant 5 is a treatment success. For child-reported depression symptoms on the RCADS, participant 5 was "clinically significant" at baseline and fell below the clinical cutoff at follow-up. Parent reported Overall Stress on the SDQ decreased from abnormal scores at baseline to normal scores at follow-up. Results also demonstrate that 
Participant 5 no longer met diagnostic criteria for any DSM-IV disorder at follow-up. Parentreported functioning also indicated a decline in functional impairment from baseline to follow-up no longer indicating definite impairment.

\section{Participant 6}

\section{Symptoms and diagnosis domain.}

Child report measure. Participant 6 completed nine total assessments (3 baseline, 3 treatment, and 3 follow-up). The results of Participant 6's self-reported levels of depression using the Total Depression scale of the Revised Child Anxiety and Depression Scale (RCADS) and represented as overall $T$-scores are presented in Figure 17. These results indicate that Participant 6 experienced his highest level of depression $(T$-score $=64)$ at the first baseline assessment. Participant 6's Total Depression $T$-scores mean for each assessment phase declined over baseline (mean $T$-score $=58.3)$, treatment (mean $T$-score $=43.6$ ), and follow-up (mean $T$-score $=36)$. 


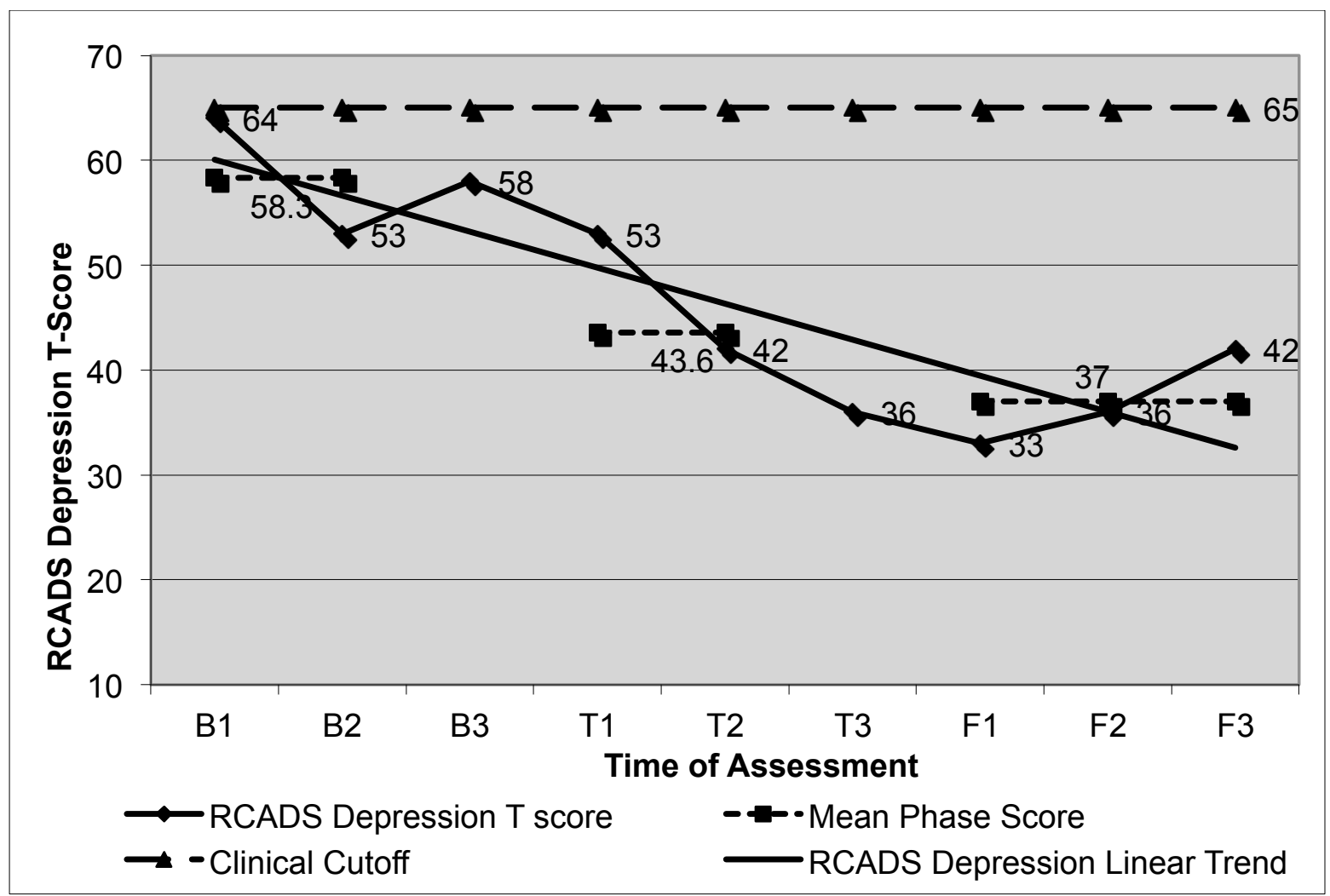

Figure 17. RCADS Depression T-scores across baseline, treatment, and follow-up for participant 6.

The results also indicate a visually significant stable, linear trend of decreasing depression symptoms from the first baseline assessment to the last follow-up. Results indicate Participant 5's Depression $T$-scores remained below the clinical range throughout baseline, treatment, and follow-up assessments. 


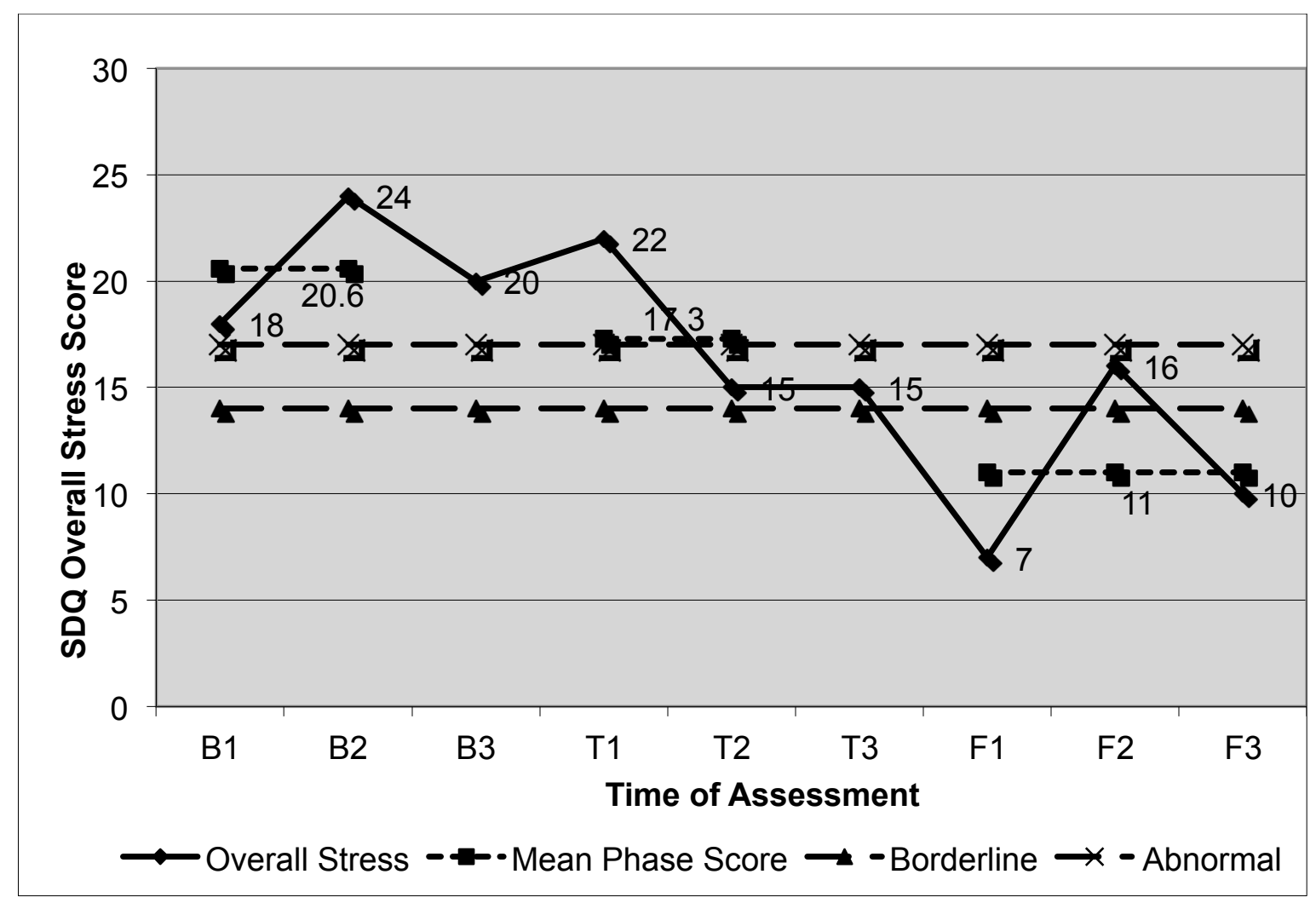

Figure 18. SDQ Overall Stress scores across baseline, treatment, and follow-up for participant 6.

Parent report measures. The results of Participant 6's parent report of levels of overall stress using the Symptoms and Difficulties Questionnaire (SDQ) and represented as scale scores are presented in Figure 18. The results indicate that Participant 6's SDQ scale scores decreased from a mean score of 20.6 at baseline down to 17.3 at treatment, and declined to a mean of 11 for the follow-up phase. Participant 6's parental reports of Overall Stress indicate her scores were in the abnormal range through the baseline phase (24 to 18) and the first treatment assessment (22). Her Overall Stress scores then decreased into the borderline range for the final two treatment assessments, both a score of 15. Participant 6's scores decreased to a low of 7 for the first follow-up assessment, increased to 16 for the second follow-up assessment, and then decreased to 10, into the normal range of scores. 
These results reveal a visually significant decrease in parental report of overall stress from baseline to follow-up. Parent reported overall stress scores were in the normal range at the final follow-up.

The initial diagnostic interview using the parent report version of the K-SADS-PL indicated that Participant 6's behavior reached criteria for minor depression, Enuresis, and ADHD. These were the only disorder for which her behaviors reached diagnostic criteria at the initial baseline assessment. Results indicate that Participant 6 did not reach diagnostic criteria for minor depression or Enuresis at the first follow-up assessment, but did meet diagnostic criteria for ADHD. At the third follow-up, Participant 6 met diagnostic criteria for ADHD and Enuresis, but did not meet diagnostic criteria for minor depression.

\section{Functioning Domain.}

Parent report measure. The results of Participant 6's parental report of functional impairment on the Brief Impairment Scale (BIS) and represented as scale scores are displayed in Figure 19. The results demonstrate that Participant 6's BIS total scale scores remained relatively stable during the baseline phase (scale scores ranging from 31 to 25) with a mean scale score of 27.3, and the first treatment assessment (28), and then began to decline from the second treatment assessment (treatment mean scale score $=19.6$ ) to the first followup assessment (11). Parental report indicated an increase in BIS total scores at the second follow-up (scale score $=21$ ) before declining in the final follow-up (follow-up mean scale score $=14.3$ ). Further, these results reveal a visually significant linear decrease in parental report of functional impairment from baseline to follow-up. 


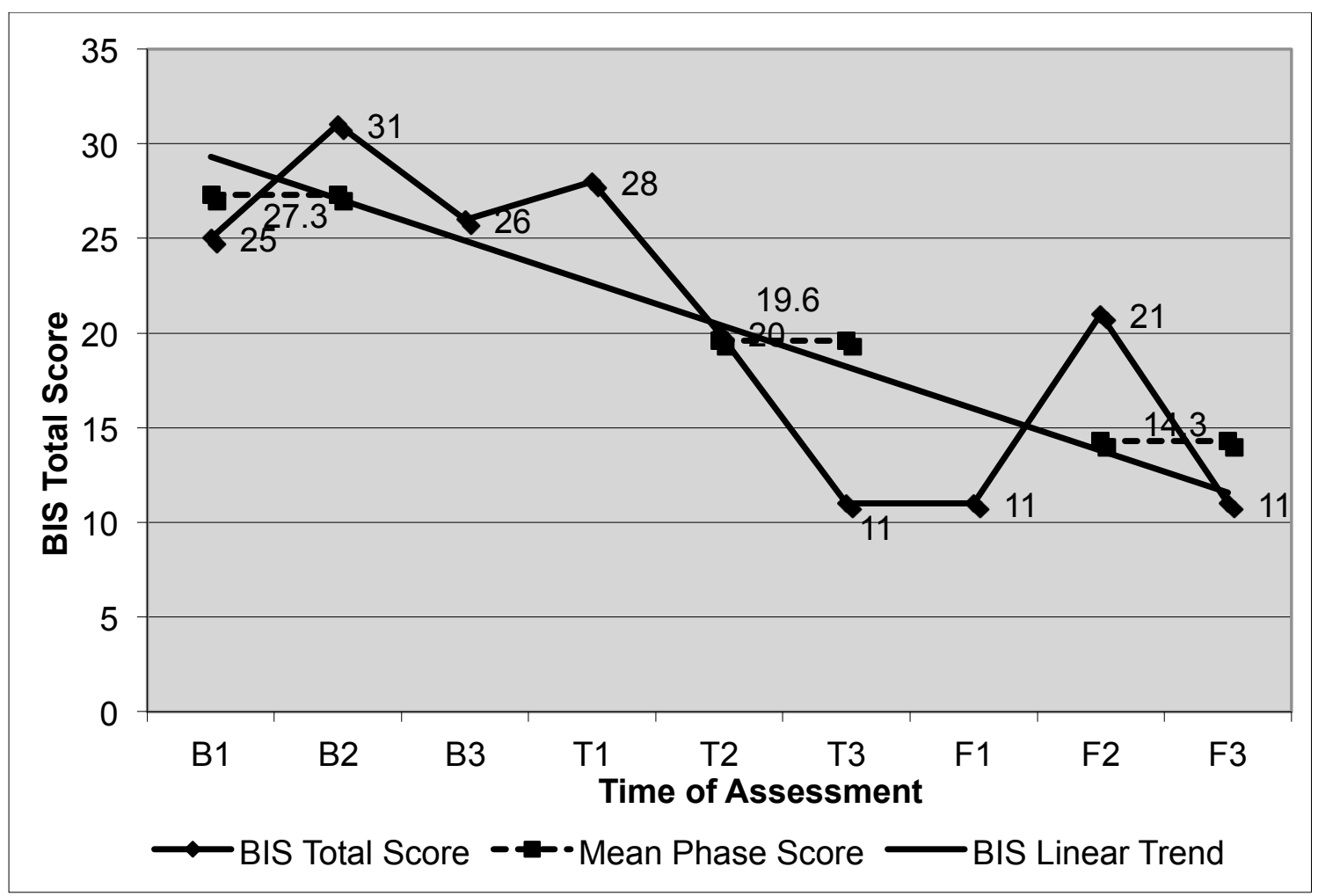

Figure 19. BIS total scale scores across baseline, treatment, and follow-up for participant 6.

Overall, Participant 6's treatment outcomes are mixed. For child-reported depression symptoms on the RCADS, participant 6 was "clinically significant" at baseline and fell below the clinical cutoff at follow-up. Parent reported Overall Stress on the SDQ decreased from abnormal scores at baseline to normal scores at follow-up. Participant 6 did meet diagnostic criteria for ADHD and enuresis at follow-up, although he no longer met criteria for minor depression. Parent-reported functioning also indicated a decline in functional impairment from baseline to follow-up, although the BIS mean score (14.3) at follow up still indicated definite impairment.

\section{Participant 7}

\section{Symptoms and diagnosis domain.}

Child report measure. Participant 7 completed eight total assessments ( 3 baseline, 1 treatment, and 4 follow-up). The results of Participant 7's self-reported levels of anxiety 
using the Total Anxiety scale of the Revised Child Anxiety and Depression Scale (RCADS) and represented as overall $T$-scores are presented in Figure 20. These results indicate that Participant 7 experienced her highest level of anxiety $(T$-score $=62)$ at the last follow-up assessment. Participant 7's Total Anxiety $T$-scores mean for each assessment phase increased over baseline (mean $T$-score $=47.6)$, treatment $($ mean $T$-score $=48)$, and follow-up $($ mean $T$ score $=51)$. The results also indicate a linear trend towards increasing anxiety symptoms from baseline to follow-up.



Figure 20. RCADS T-scores across baseline, treatment, and follow-up for participant 7. While Participant 7's results show a general increase in Total Anxiety $T$-scores from baseline to treatment and treatment to follow-up, Participant 7's Anxiety $T$-scores never reach clinically significant levels.

Parent report measures. The results of Participant 7's scores of overall stress using the Symptoms and Difficulties Questionnaire (SDQ) parent-report and represented as overall 
scale scores are presented in Figure 21. The results indicate that Participant 7's SDQ scores remained relatively stable during baseline and treatment phase. At the second follow-up the Overall Stress score increased to 17 and then decreased to 9 at the third follow-up before increasing again to 15. The results of Participant 7's parental reports graph indicates Overall Stress scores were in the borderline range from the first baseline assessment to the first follow-up assessment and increased to the abnormal range at the second follow-up. Participant 7's Overall Scores were in a normal range at the third follow-up assessment and returned to the borderline range in the final follow-up. These results reveal a slight downward trend in parental report of overall stress from baseline to follow-up.

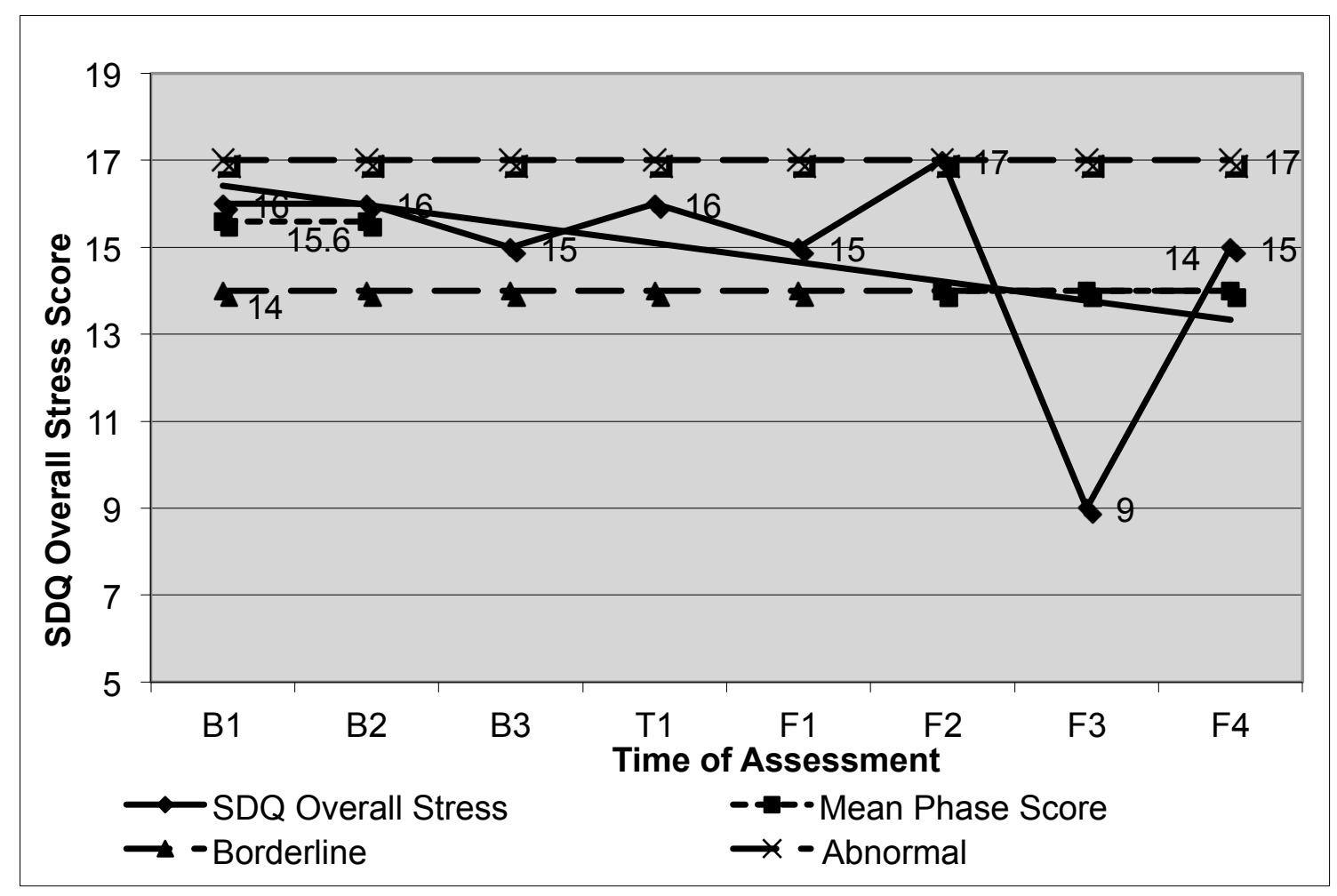

Figure 21. SDQ Overall Stress scores across baseline, treatment, and follow-up for participant 7. 
The initial diagnostic interview using the parent report version of the K-SADS-PL indicated that Participant 7's behavior reached DSM-IV criteria for Simple Phobia, Social Phobia, and Generalized Anxiety Disorder. Results indicate that Participant 7 only met criteria for Simple Phobia and Social Phobia at the first follow-up assessment. The diagnostic interview at the second follow-up assessment indicated that Participant 7 only met criteria for Simple Phobia and at the final follow-up assessment Participant 7 met criteria for Simple Phobia and Social Phobia.

\section{Functioning Domain.}

Parent report measure. The results of Participant 7's parent report of functional impairment on the Brief Impairment Scale (BIS) and represented as scale scores are displayed in Figure 22. The results demonstrate that Participant 7's BIS total scale scores declined from the first baseline to the final follow-up assessment. Parental report indicates a decrease in the BIS total scores mean from baseline (mean scale score $=12.6$ ) to follow-up (mean scale score $=9.25)$. Further, these results reveal a visually significant linear decrease in parental report of functional impairment from baseline to follow-up. 


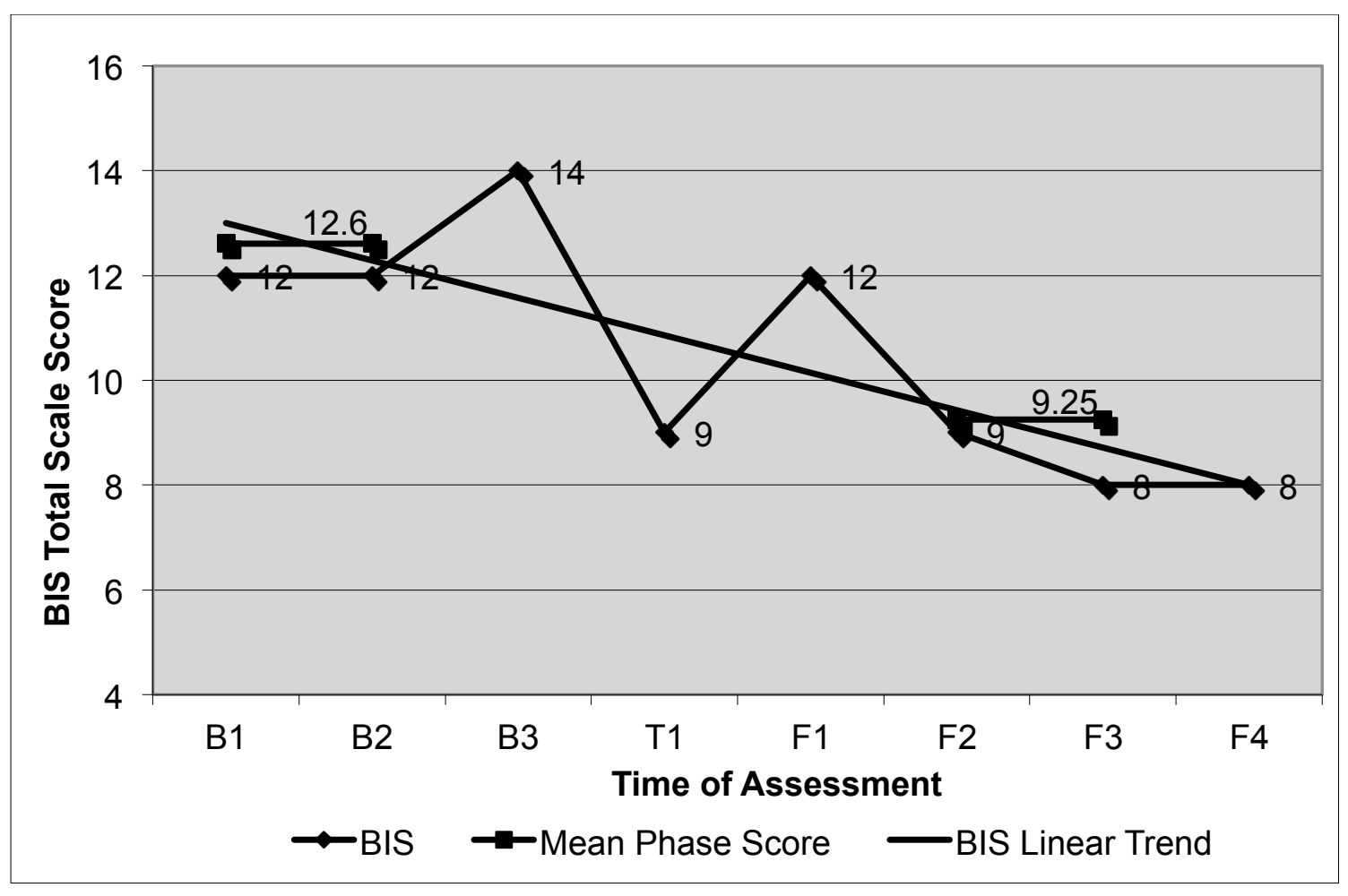

Figure 22. BIS total scale scores across baseline, treatment, and follow-up for participant 7.

Overall, Participant 7 has poor outcomes, suggesting treatment was not successful. Child-reported anxiety symptoms on the RCADS, although never "clinically significant," increased from baseline to follow-up. Parent reported Overall Stress on the SDQ remained in the borderline range at follow-up. Although Participant 7 no longer met diagnostic criteria for generalized anxiety disorder at follow up, a diagnosis of simple phobia and social phobia were present at follow-up. Parent-reported functioning indicated a slight decline in functional impairment from baseline to follow-up.

\section{Participant 8}

\section{Symptoms and diagnosis domain.}

Child report measure. Participant 8 completed three total assessments (1 baseline, 1 treatment, and 1 follow-up). The results of Participant 8's self-reported levels of depression using the Total Depression scale of the Revised Child Anxiety and Depression Scale 
(RCADS) and represented as overall $T$-scores are presented in Figure 23. These results indicate that Participant 8 experienced her highest level of depression $(T$-score $=75)$ at baseline. The results also indicate a linear trend towards declining depression symptoms from baseline to follow-up. Participant 8's indicate that her Depression $T$-scores reached clinical significance at baseline. Participant 8's $T$-scores were no longer clinically significant at treatment or follow-up.



Figure 23. RCADS T-scores across baseline, treatment, and follow-up for participant 8 .

Parent report measures. The results of Participant 8's scores of overall stress using the Symptoms and Difficulties Questionnaire (SDQ) parent-report and represented as overall scale scores are presented in Figure 24. The results indicate that Participant 8's SDQ scores declined from baseline to treatment. The results of Participant 8's parental reports graph indicates Overall Stress scores were in the abnormal range at the baseline assessment and 
decreased to the borderline range at follow-up. These results reveal a downward trend in parental report of overall stress from baseline to follow-up.

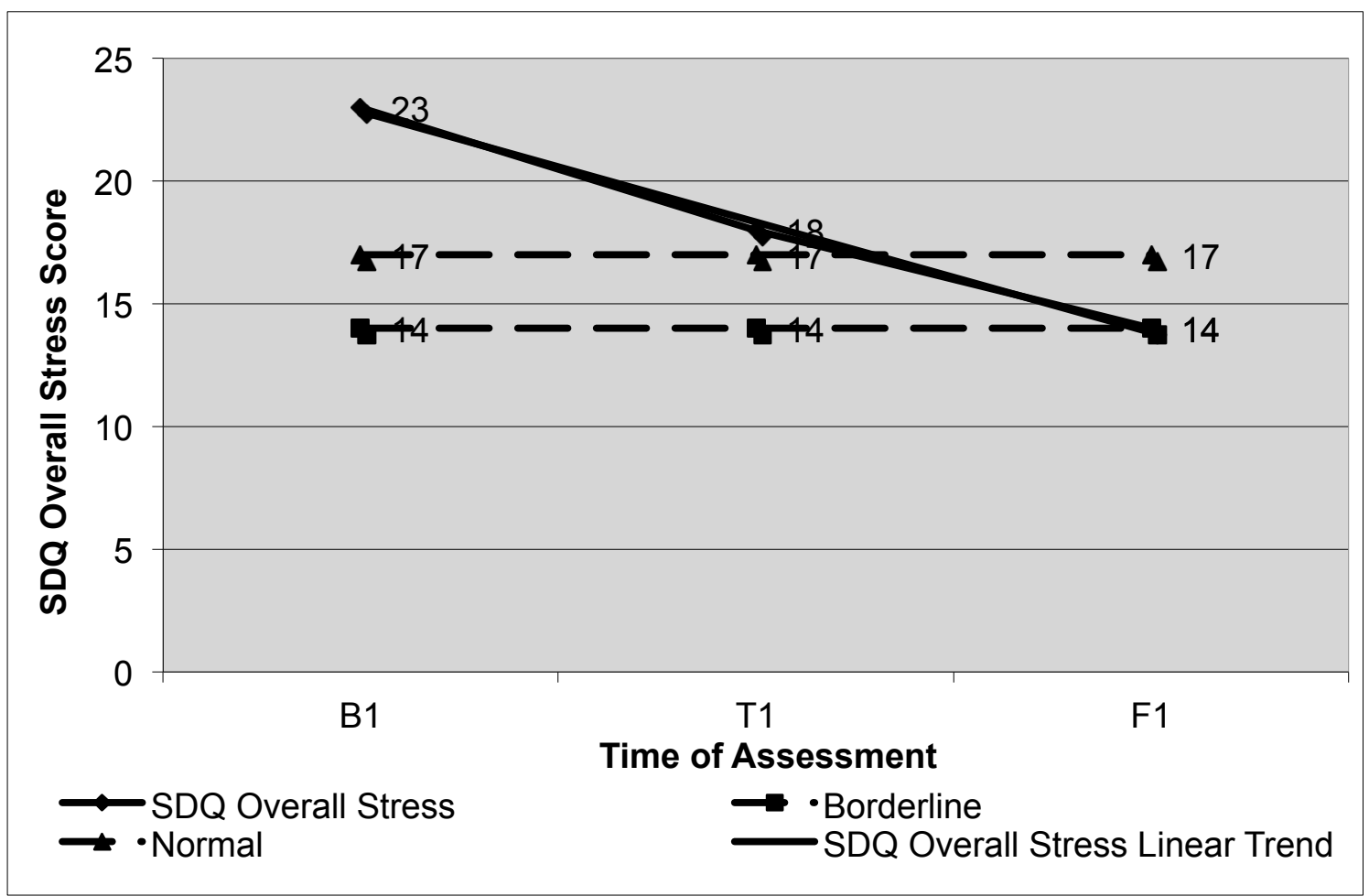

Figure 24. SDQ Overall Stress scores across baseline, treatment, and follow-up for participant 8 .

The initial diagnostic interview using the parent report version of the K-SADS-PL indicated that Participant 8's behavior reached DSM-IV criteria for Major Depression. This was the only disorder for which her behaviors reached diagnostic criteria at the initial baseline assessment. Results indicate that Participant 8 did not reach diagnostic criteria for Major Depression, or for any other DSM-IV disorder at any point during the follow-up assessment.

\section{Functioning Domain.}


Parent report measure. The results of Participant 8's parental report of functional impairment on the Brief Impairment Scale (BIS) and represented as scale scores are displayed in Figure 25. The results demonstrate that Participant 8's BIS total scale scores decline from baseline to the follow-up assessment. Further, these results reveal a linear decrease in parental report of functional impairment from baseline to follow-up.



Figure 25. BIS total scale scores across baseline, treatment, and follow-up from participant 8.

Overall, Participant 8's treatment outcomes are mixed. For child-reported depression symptoms on the RCADS, participant 8 was "clinically significant" at baseline and fell below the clinical cutoff at follow-up. Parent reported Overall Stress on the SDQ decreased from abnormal scores at baseline to normal scores at follow-up. Participant 8 no longer met diagnostic criteria for any DSM-IV disorder. Parent-reported functioning also indicated a 
slight decline in functional impairment from baseline to follow-up, although the BIS mean score (20) at follow up still indicated definite impairment.

\section{Summary}

An overall summary of participant outcomes and whether they support treatment effectiveness is displayed in Table 5. An upward arrow indicates that evidence supports treatment effectiveness, a sideways arrow indicates that evidence on treatment effectiveness is mixed, and a downward arrow indicates that treatment effectiveness was not supported. In order to be considered a success, parent and/or child report measures needed to indicate a significant decrease in primary diagnosis symptoms, a decline in functional impairment, and no diagnosis at follow-up. A participant is classified as mixed if treatment outcomes indicate either a decline in parent and/or child reported symptoms, and/or a decline in functional impairment, and/or not meeting diagnostic criteria at follow-up, but not all three. Finally, a classification of not successful is made if parent and child report measures failed to indicate a decline in primary diagnosis symptoms or indicate an increase in symptoms, if functional impairment did not decline or increased, and if the participant still met diagnostic criteria for all or some of their intake diagnoses at follow-up.

Table 4.

Summary of Participant Outcomes and Treatment Effectiveness for Participant 2 to 8.

\begin{tabular}{|c|c|c|c|c|c|c|c|c|}
\hline $\begin{array}{c}\text { Participant } \\
\text { Number }\end{array}$ & $\begin{array}{c}\text { \# of } \\
\text { sessions }\end{array}$ & $\begin{array}{c}\text { Treatment } \\
\text { Outcome }\end{array}$ & $\begin{array}{c}\text { Symptoms } \\
- \text { Child } \\
\text { Report }\end{array}$ & $\begin{array}{c}\text { Symptoms } \\
- \text { Parent } \\
\text { Report }\end{array}$ & $\begin{array}{c}\text { Diagnosis at } \\
\text { Intake }\end{array}$ & $\begin{array}{c}\text { Diagnosis at } \\
\text { Follow-up }\end{array}$ & Functioning & Therapist \\
\hline 2 & 17 & Success & $\Uparrow$ & $\Uparrow$ & $\begin{array}{c}\text { Social } \\
\text { Phobia } \\
\text { Diagnosis }\end{array}$ & $\Uparrow$ & No \\
\hline
\end{tabular}




\begin{tabular}{|c|c|c|c|c|c|c|c|c|}
\hline \multicolumn{8}{|c|}{ Table 4 (continued) } & \multirow[b]{2}{*}{ A } \\
\hline 3 & 12 & $\begin{array}{c}\text { Not } \\
\text { Successful }\end{array}$ & $\Rightarrow$ & $\Downarrow$ & $\begin{array}{c}\text { Minor } \\
\text { Depression, } \\
\text { ADHD, } \\
\text { ODD }\end{array}$ & ADHD & $\Downarrow$ & \\
\hline 4 & 12 & Success & $\Uparrow$ & $\Uparrow$ & $\begin{array}{c}\text { Panic } \\
\text { Disorder, } \\
\text { Separation } \\
\text { Anxiety } \\
\text { Disorder }\end{array}$ & $\begin{array}{c}\text { No } \\
\text { Diagnosis }\end{array}$ & $\Uparrow$ & $\mathrm{B}$ \\
\hline 5 & 8 & Success & $\Uparrow$ & $\Uparrow$ & $\begin{array}{c}\text { Minor } \\
\text { Depression }\end{array}$ & $\begin{array}{c}\text { No } \\
\text { Diagnosis }\end{array}$ & $\Uparrow$ & B \\
\hline 6 & 14 & Mixed & $\Uparrow$ & $\Uparrow$ & $\begin{array}{c}\text { Minor } \\
\text { Depression, } \\
\text { Enuresis, } \\
\text { ADHD }\end{array}$ & $\begin{array}{c}\text { Enuresis, } \\
\text { ADHD }\end{array}$ & $\Uparrow$ & $\mathrm{A}$ \\
\hline 7 & 2 & $\begin{array}{c}\text { Not } \\
\text { Successful }\end{array}$ & $\Downarrow$ & $\Downarrow$ & $\begin{array}{c}\text { Simple } \\
\text { Phobia, } \\
\text { Social } \\
\text { Phobia, } \\
\text { GAD }\end{array}$ & $\begin{array}{c}\text { Simple } \\
\text { Phobia, } \\
\text { Social } \\
\text { Phobia }\end{array}$ & $\Rightarrow$ & $\mathrm{A}$ \\
\hline 8 & 8 & Mixed & $\Uparrow$ & $\Uparrow$ & $\begin{array}{c}\text { Major } \\
\text { Depression }\end{array}$ & $\begin{array}{c}\text { No } \\
\text { Diagnosis }\end{array}$ & $\Longrightarrow$ & B \\
\hline
\end{tabular}

\section{Discussion}

In the past few years, primarily due to a growing evidence base of efficacious mental health treatments for youth, optimism in the field about transporting evidence based treatments into multiple service settings has increased (Weisz et al., 1995; Chorpita \& Daleiden, 2009. However, issues with transportability and implementation have been identified and outcomes from recent attempts to study these treatments outside of research settings have been mixed (Southam-Gerow et al., 2010; Weisz et al., 2009). This initial pilot transportability study aimed to help move the field further down the path to wide-spread 
dissemination by implementing a partnership model to adapt evidence-based treatments (EBTs) in a community mental health setting using single case design. The goals were to (a) demonstrate that adapted EBTs would lead to improvements in client symptoms and functioning and (b) provide data to help make adaptations to the EBTs for the third phase of the project. The present study addressed these goals by enrolling eight participants with various diagnoses (i.e., depression, enuresis, ADHD, simple phobia, social phobia, GAD, separation anxiety disorder, panic disorder, ODD) into a single case series. Measurements were made at baseline, treatment, and follow-up across two domains, (a) symptoms and diagnosis domain (b) and functioning domain. Both parent report and child self-report measures were administered.

In this next section I will summarize and interpret the results of the single case series as well as discuss the implications of the study outcomes. Next, I will discuss the limitations of the current study. Finally, future directions will be suggested and the next phase of the ADAPT study will be described.

\section{Interpretation and Summary of Results}

Altogether, the results of the present study illustrate mixed support for the adapted treatment in a community mental health setting as evidenced by differential treatment outcomes among the seven participants in Table 5. Treatment outcomes for three

participants (i.e., 2, 4, 5) were considered a success, whereas two were deemed not successful (i.e., 3, 7) and two participant outcomes demonstrated mixed support (i.e., 6, 8). Due to unstable baseline trends in some outcome measures and/or some participants not appearing in the clinical range of scores at baseline, it was difficult to determine clear treatment effects and visual inspection of results became more subjective. However, criteria have been 
suggested to strengthen the interpretation of clinically significant change such as the elimination of the presenting problem and displaying normative levels of functioning at follow-up (Jacobson \& Truax, 1991). In the current study, success was not only determined by trend in the data but also a change from the clinical range of scores to the normal range of scores on certain measures from baseline to follow-up, as well as the absence of diagnosis at follow-up therefore strengthening interpretation of results.

Why did some youth evidence improved outcomes whereas others demonstrated mixed or poorer outcomes? Although interpretation of results is limited due to the nature of single case design, the data raise two possible hypotheses as to why the study goals were not fully supported: (1) cases where participants presented no or fewer comorbidities were more successful in treatment, and (2) differences in treatment dose led to differing outcomes among the participants.

Results suggest that treatment was more successful with participants who only met diagnostic criteria for one or two DSM-IV disorders versus those participants with multiple diagnoses. As stated previously, youth with multiple comorbidities may be more difficult to treat than youth that present with only a single DSM-IV disorder (Kazdin \& Whitley, 2006). EBTs have also been developed in research settings to usually focus on one DSM-IV disorder, hence leaving a gap in the evidence base for treatments that work for children with multiple comorbidities. The participants in our study who were considered a treatment success presented at intake with what may be considered less complex clinical pictures than the participants for whom treatment was less successful. For example, two of the cases with better outcomes (participant 2 and 5) met criteria for a single DSM-IV internalizing disorder and a third case with a strong outcome (participant 4) met criteria for two DSM-IV 
internalizing disorders. The participants with the poorest outcomes, on the other hand, had what appear to be more complex clinical presentations when judged by presence of DSM-IV disorders. Both cases with the poorest outcomes (participant 3 and 7) met criteria for three DSM-IV disorders, one of which had a mixed internalizing and externalizing diagnostic picture. The two participants with mixed outcomes provide some additional support to the hypothesis that multiple comorbidities negatively affected treatment outcomes because one participant presented at intake with no comorbidities (participant 8), whereas one participant presented at intake with three comorbid disorders (participant 6). In participant 8's case, symptoms and diagnosis domain measures indicated treatment gains, whereas the functioning domain measure demonstrated continued functional impairment at follow-up. In other words, of the two cases with mixed outcomes, the least complex clinical case, participant 8 , had a better outcome than the more complex case, participant 6 . It is worth noting though that participant 6, despite demonstrated treatment gains in symptoms and functioning, continued to demonstrate behavior indicative of two DSM-IV diagnoses at all follow-up assessments, suggesting the possibility that multiple comorbidities decreased treatment effects. Taken together, these results suggest that complexity and/or severity of the presenting problem, and in particular comorbid externalizing symptomatology, may account for the diminished treatment gains observed in the present study, a result consistent with past work (Rohde, Clarke, Mace, Jorgensen, \& Seeley, 2004; Garcia et al., 2010).

Externalizing symptomatology as a moderator of treatment has also been illustrated in the current child treatment literature. In an randomized controlled study of cognitivebehavioral treatment for adolescents with major depression and comorbid conduct disorder, treatment was found to be effective at reducing depressive symptoms but not effective at 
reducing symptoms of conduct disorder (Rohde et al., 2004). In an OCD treatment study, where youths were randomized to one of three treatment groups (CBT-only, SSRI-only, CBT/SSRI), outcomes indicated that youth with higher levels of externalizing symptoms fared worse across all treatment conditions relative to peers with lower levels of externalizing symptoms (Garcia et al., 2010). In contrast, treatment studies for youth anxiety have indicated no treatment differences for youth with comorbid externalizing symptomatology (Flannery-Schroeder, Suveg, Safford, Kendall, \& Webb, 2004; Verduin \& Kendall, 2003). However, both of these study samples had few youth with comorbid disruptive behavior disorders and evidence has demonstrated that anxiety research clinic populations have a significantly lower incidence of comorbid externalizing disorders than community clinic samples (Southam-Gerow et al., 2006; 2008). Overall, the treatment literature indicates support for the current study's hypothesis that externalizing symptomatology may account for decreased treatment gains.

Severity of presenting problem is another potential moderator of treatment outcomes that has been supported in the child treatment literature (Southam-Gerow et al., 2001; Berman, Weems, Silverman, \& Kurtines, 2000; Salzer, Bickman, \& Lambert, 1999). In an analysis of archival data using participant information from the Fort Bragg Evaluation Project, Salzer and colleagues (1999) found that clients who were in treatment longer had significantly more problems and more severe problems at intake. Similarly, Berman and colleagues (2000) examined predictors of exposure-based CBT for phobic and anxiety disorders in youth and found that children with a comorbid diagnosis of depression were more likely to demonstrate diminished treatment gains than children without comorbid depression. A study on youth with anxiety disorders also found that increased levels of 
mother- and teacher-reported child internalizing symptoms were significantly related with poorer treatment outcomes (Southam-Gerow et al., 2001).

Another third and final factor that may explain the mixed outcomes observed in the present study is treatment dose or length. Treatment length for participants who were considered a treatment success attended between 10 to 17 sessions with a mean of 13 , whereas participants who evidenced mixed treatment gains attended between 8 to 14 sessions, with a mean of 11 , and those who did not experience treatment gains attended between 2 to 12 sessions, with a mean of 7 sessions. The literature on treatment dose for child psychotherapy, although scant, illustrates mixed findings on the effect of dose on treatment outcomes. For example, treatment outcomes in a Parent-Child Interaction Therapy (PCIT) follow-up study indicated that parents who completed treatment experienced significantly better outcomes than parents who dropped out of treatment (Boggs et al., 2004). Important to note is that no pretreatment differences were found for demographic or clinical characteristics between the "completer" group and the "dropout" group. In a meta-analysis of child therapy treatment studies, Jensen and colleagues found that 13 out of 27 studies since 1995 demonstrated a significant difference among similarly intensive treatment groups illustrating that treatment dose makes a difference in outcomes (Jensen, Weersing, Hoagwood, \& Goldman, 2005). On the other hand, Salzer, Bickman, and Lambert found no general treatment dose effect when looking at this effect in archival data of 567 children who participated in the Fort Bragg Evaluation Project (1999). Despite these findings on the effects of treatment dose, Hoagwood (2000) stated that differences in the way "dose" is defined, measured, and analyzed disguises the fact that an important part of child treatment development, identifying the mechanisms of action, is missing (2000). To measure dose 
accurately, it is important to know why a particular therapeutic approach has its intended effect. Not until we know how treatment works can we begin to recommend at what dose it is most effective. Essentially, appropriate treatment dose could be different depending on diagnosis, treatment used, environmental factors, age, gender, etc. (Salzer et al., 1999).

\section{Implications}

The results of this study demonstrate the challenges in using EBTs in novel community clinic settings. Focus of treatment varied greatly for each participant, depending on diagnostic profile at intake as well as the therapist's case conceptualization for each case. One implication of the adaptations of the EBTs, although allowing for individualization of treatment for each youth, is that efficacy cannot be assumed due to the adaptations that were made. In addition, this study illustrates the need to consider if and how treatment dose affects treatment outcomes, especially at a time when many healthcare companies want to impose limits and restrictions on mental health services (Andrade, Lambert, \& Bickman, 2000). Research into ways of decreasing attrition among youth and families in community clinic settings could allow for further adaptations of EBTs in these settings (Miller, SouthamGerow, \& Allin, 2008). It is also important to determine the typical number of sessions needed for maximum effectiveness for a particular type of client and for a particular type of problem (Salzer et al., 1999).

Furthermore, outcomes for the symptoms and diagnosis domain suggest that cases with mixed internalizing/externalizing presentations might require further adaptation. Although treatment in mixed presentation cases followed both internalizing and externalizing EBT manuals, the focus of treatment were the internalizing disorder diagnoses. Other researchers have demonstrated alternative approaches to incorporating externalizing 
symptomatology and other comorbidities in treatment programs. The modular approach developed by Chorpita and colleagues (Chorpita, 2007; Chorpita, Daleiden, \& Weisz, 2005) allows for the "preservation of standard protocol when necessary," but would also allow for scaling and/or adaptations as needed. The modular approach to treatment consists of selfcontained "modules" that connect with other "modules," but can be used at any time throughout treatment and do not rely on a certain "module" needing to be presented before it or after it (Chorpita et al., 2005). This flexible adaptability allows for easier transportability into different settings and increased individualization for each client. Similar to the current study, the modular approach builds the treatment plan around a youth's primary diagnosis. Weiss and colleagues (Weiss, Harris, Catron, \& Han, 2003) developed an intervention designed to treat concurrent internalizing and externalizing disorders in youth called Reaching Educators, Children and Parents program (RECAP). RECAP is a combination of modified treatment techniques (coping skills training, problem-solving skills training, parent training) that were chosen to target factors believed to be responsible for maintaining both child internalizing and externalizing problems. The treatment plan is not driven by the youth's primary diagnosis, but is designed to target these three specific domains that affect both internalizing and externalizing symptoms. Continued research with treatments for children with comorbid disorders is needed to assess why and how externalizing comorbidity affects outcomes and how best to address cases in which the client has multiple problems.

The present results also illustrated that all participants, regardless of overall treatment gains or lack thereof, demonstrated some improvement over the course of treatment whether in symptoms and diagnosis or functioning. This contributes to the current literature that recommends assessing not only the symptom and diagnosis domain in treatment studies, but 
also other domains (e.g., functioning) to get a more complete picture of how the youth is responding to treatment (Becker, Chorpita, \& Daleiden, 2011; Hoagwood et al., 1996; Weiss et al., 2003). Functional impairment is a necessary criterion for diagnosing a youth with a DSM-IV diagnosis (APA, 2000).

In short, this study represents an initial step in a larger project aimed at testing the utility of a partnership model for adapting EBTs for youth in a community clinic setting. The findings of this study suggest some useful hypotheses to test in a larger scale study.

Specifically, participants who did not present with a comorbid disorder benefited from treatment elements not ordinarily found in one standard manual (e.g., communication skills training for the family in treatment for depression).

\section{Limitations}

There are limitations to this study to consider. The most pressing concern is the limited ability to generalize findings to other populations and/or other clinic settings. Kazdin (1982) stated that generality is not necessarily a problem for single case studies in that visual inspection detects dramatic intervention effects that may be more likely to generalize than more subtle effects detected by between-group designs. On the other hand, difficulty in assessing elements of treatment that may relate to generality, such as therapist (e.g., treatment adherence, treatment competence) and/or participant variables (e.g., gender, age) are more appropriately evaluated in a between-group design. However, a single case design study that is used as a preliminary test of a new or adapted treatment could allow researchers to develop hypotheses about the study results and test those hypotheses in a larger group design. Another limitation that affected generalizability was the heterogeneity of youths, 
including multiple comorbidities, which could have undermined treatment focus on the youth's primary diagnostic problem.

The use of randomization to determine the number of baseline assessments for each participant also limited interpretation of results. Performance during baseline assessments is used to predict how the youth may continue to behave without intervention so it is important to demonstrate a stable rate of performance in this phase (Kazdin, 1982). In some outcome measures (e.g., Figure 1), a participant may have demonstrated a baseline trend in the same direction that the intervention produced, hence interfering with the ability to determine clear treatment effects. In order to control for trends in baseline, some have suggested a data based baseline determination procedure where the treatment phase is only introduced when the data show no trend in the baseline phase (Koehler \& Levin, 1998). Another option suggested is to randomize intervention introduction once a stable baseline has been achieved. Statistical analyses also may allow for the evaluation of effects by accounting for the initial trend in the baseline data (Kazdin, 1982). In the current study, the clinic required that the youth wait no longer than one month for treatment, hence not allowing for a data based baseline determination.

The issue of clinical significance was another challenge that arose in interpretation of results. Due to the shortened baseline period, certain measures (e.g., CBCL) were administered at shorter intervals than recommended for re-administration. For example, it is recommended that the CBCL only be administered every one to two months, but it was administered every one to two weeks during the baseline phase of the current study (Achenbach \& Rescorla, 2004). Due to unstable baseline trends in some outcome measures and/or some participants not appearing in the clinical range of scores at baseline, it was 
difficult to determine clear treatment effects and visual inspection of results became more subjective. Criteria have been suggested to strengthen the interpretation of clinically significant change such as the elimination of the presenting problem and displaying normative levels of functioning at follow-up (Jacobson \& Truax, 1991).

Another limitation or challenge that was faced throughout the study was engaging participants in recruitment and continuous assessment. Inclusion criteria for the study initially indicated recruiting participants with an internalizing disorder and comorbid externalizing symptomatology, unfortunately due to time constraints and a lack of available cases, children who only had internalizing behavior problems were included as well. Last, participants and their families often had trouble attending the multiple and sometimes lengthy assessments, was difficult, especially once a participant had terminated treatment. Due to this, assessments did not always occur according to schedule. A related issue that limited the study findings was missing data. Due to difficulty scheduling assessments, some participants did not have any data for certain assessment points and/or assessments were shortened and not all measures administered due to participant time constraints. Creating multiple avenues for data collection including an online assessment tool that would allow families to complete measures at their convenience could increase engagement. Another option could be having the therapists administer certain measures to the youth and family at the time of their treatment session, thus eliminating the need to come back to the clinic for multiple assessment sessions. The measurement model also shifted throughout the single case study, which caused us to have measures for certain assessment phases and not others making data interpretation difficult. 
Finally, adapting efficacious treatments runs the risk of removing or diluting the important ingredients. Since different treatment elements were being used out of different manuals in a non-standard sequence, the treatments were not the same ones that were efficacious in research settings. The therapists at the clinic were allowed to individualize the treatment based on their conceptualization of what the youth and his or her family needed, in consultation with the study supervisor. This limited our ability to interpret findings in relation to which treatment elements worked and did not work and how best to sequence certain treatment elements. Although used in a non-standard fashion, the treatment plans were guided by a reliance on EBTs, and the quality of the sequence and treatment delivery was monitored in supervision.

\section{Future Directions}

The results of the study suggest many important directions for future scientific work related to the transportability of EBTs into community clinics and other service clinic settings. One important issue is how best to adapt treatments to ensure positive treatment outcomes for youth with comorbid disorders, especially youth that present with comorbid internalizing and externalizing symptomatology. These results suggest that the tested method of supplementing EBTs for anxiety or depression with elements from an EBT for externalizing disorders was not effective. Similar to RECAP (Weiss et al., 2003) described earlier, perhaps using a treatment that focuses more generally on factors that perpetuate both internalizing and externalizing symptoms would lead to better outcomes for youth with comorbid presentations in community clinics. Studies could also increase representation of youth with externalizing comorbidity to determine how best adaptations could be made to increase treatment gains in this population. 
Future studies could also consider the cost-effectiveness of training community therapists on the adapted treatment. The current study uses an expert in the community to train the community therapists and supervise all of the cases; unfortunately, this opportunity is not always available for many community clinics so accessibility to training tools and therapy manuals is necessary for the widespread dissemination of these adapted treatments into multiple settings (Addis, 2002).

Another suggestion for measuring successful adaptation of treatments in community clinics would be to measure therapist competence and adherence with the adapted EBTs. For the next phase of the current study, the open trial phase, a modular approach to treatment, as described earlier, will be implemented. Most current EBTs are considered as "integral" approaches to treatment, defined as parts functioning as a single whole creating a high level of interdependence among each part, therefore although these "integral" manuals can be divided into individual sessions, the sessions do not stand alone and ordinarily have to be presented to a client in a certain order. This flexible adaptability of the modular approach may allow for easier transportability, increased individualization for each client, and is more comprehensible for therapists since one "module" can be studied at a time. The next phase of ADAPT will allow us to test the hypotheses raised in the current study. The treatment approach in the open trial phase allows for a better integrated approach to deal with youth with comorbid disorders. Feedback from the therapists' experience in the current study also help to guide efforts for the next phase, for example, in the addition of new treatment modules (e.g., communication, emotion regulation). Using a more "formal" approach to adaptation might allow for more robust effects in future studies, allowing better measurement of client and therapist factors that affect treatment outcome. Knowing these factors could 
lead to better adaptations in treatment as well as increased implementation for diverse populations and clinic settings.

Overall, the results of the single case series provided preliminary, though somewhat mixed, support for both the adapted treatment approach and the partnership model that is part of the broader ADAPT project. Continued research in (1) how best to address comorbid externalizing symptoms and/or severity of diagnosis and (2) how to improve retention and increase treatment dose, will eventually lead to better adaptations of EBTs and allow for increased implementation of EBTS with diverse populations and settings. 


\section{List of References}

Achenbach, T. M., \& Rescorla, L. A. (2000). Manual for the ASEBA School-Age Forms And Profiles. Burlington, VT: University of Vermont, Research Center for Children, Youth, \& Families.

Achenbach, T.M., \& Rescorla, L.A. (2004). The Achenbach System of Empirically Based Assessment (ASEBA) for ages 1.5 to 18 years. In M.E. Maruish (Ed.), The use of psychological testing for treatment planning and outcome assessment (3rd ed., Vol. 2). Mahwah, NJ: Lawrence Erlbaum Associates.

Addis, M. E., Hatgis, C., Krasnow, A. D., Jacob, K., Bourne, L., \& Mansfield, A. (2004). Journal of consulting and clinical psychology, 72(4), 625-35. doi: 10.1037/0022006X.72.4.625.

Addis, M. E., \& Krasnow, A. D. (2000). A national survey of practicing psychologists' attitudes toward psychotherapy treatment manuals. Journal of Consulting \& Clinical Psychology, 68, 331-339.

Adelman, H. S., \& Taylor, L. (2004). Mental health in schools: A shared agenda. Emotional \& Behavioral Disorders in Youth, 4, 59-62.

American Psychiatric Association. (2000). Diagnostic and statistical manual of mental disorders (Revised 4th ed.). Washington, DC: Author.

American Psychological Association. (2005). American Psychological Association Statement Policy Statement on Evidence-Based Practice in Psychology, 1-3.

Andrade, A. R., Lambert, E. W., \& Bickman, L. (2000). Dose effect in child psychotherapy: Outcomes associated with negligible treatment. Journal of the American Academy of Child \& Adolescent Psychiatry, 39, 161-168.

Angold, A., \& Costello, E. J. (1993). Depressive comorbidity in children and adolescents: Empirical, theoretical, and methodological issues. American Journal Of Psychiatry, 150, 1779-1791.

Angold, A., Erkanli, A., Silberg, J., Eaves, L., \& Costello, E. J. (2002). Journal Of Child Psychology And Psychiatry, 8, 1052-1063.

Arnold, L. E., Elliott, M., Sachs, L., Bird, H., Kraemer, H. C., Wells, K. C., et al. (2003). Journal of Consulting and Clinical Psychology, 71(4), 713-727. doi: 10.1037/0022006X.71.4.713.

Barkley, R. A. (1997). Defiant children: A clinician's manual for assessment and parent training $\left(2^{\text {nd }}\right.$ ed. $)$. New York: Guilford Press. 
Barrington, J., Prior, M., Richardson, M., \& Allen, K. (2005). Behaviour Change, 22(1), 2943. doi: 10.1375/bech.22.1.29.66786.

Becker, K. D., Chorpita, B. F., \& Daleiden, E. L. (2011). Improvement in Symptoms Versus Functioning: How Do Our Best Treatments Measure Up? Administration and policy in mental health. doi: 10.1007/s10488-010-0332-x.

Berman, S., Weems, C., Silverman, W., \& Kurtines, W. (2000). Predictors of outcome in exposure-based cognitive and behavioral treatments for phobic and anxiety disorders in children. Behavior Therapy, 31, 713-731. doi: 10.1016/S0005-7894(00)80040-4.

Bernstein, G. A. \& Borchardt, C. M. (1991). Anxiety disorders of childhood and adolescence: A critical review. Journal of the American Academy of Child \& Adolescent Psychiatry, 30, 519-532.

Bird, H., Canino, G., Davies, M., Ramirez, R., Chavez, L. Duarte, C., et al. (2005). The brief impairment scale (BIS): A multidimensional scale of functional impairment for children and adolescents. Journal of the American Academy of Child \& Adolescent Psychiatry, 44, 699-707.

Bird H., Shaffer D., Fisher P., et al. (1993). The Columbia impairment scale (CIS): Pilot findings on a measure of global impairment for children and adolescents. International Journal of Methods in Psychiatric Research, 3, 167-176.

Bird, H. R., Gould, M. S., \& Staghezza, B. M. (1993). Patterns of diagnostic comorbidity in a community sample of children aged 9 through 16 years. Journal of the America Academy of Child \& Adolescent Psychiatry, 37, 35-49.

Boggs, S. R., Eyberg, S. M., \& Edwards, D. L. (2004). Outcomes of parent-child interaction therapy: A comparison of treatment completers and study dropouts one to three years later. Child \& Family Behavior Therapy, 26, 1-22. doi:10.1300/J019v26n04_01.

Borduin, C. M., Mann, B. J., Cone, L. T., Henggeler, S. W., Fucci, B. R., Blaske, D. M., et al. (1995). Multisystemic treatment of serious juvenile offenders: Long-term prevention of criminality and violence. Journal of Consulting and Clinical Psychology, 63, 569-578.

Brent, D. A., Holder, D., Kolko, D., Birmaher, B., Baugher, M., Roth, C., et al. (1997). A clinical psychotherapy trial for adolescent depression comparing cognitive, family, and supportive therapy. Archives of General Psychiatry, 54(9), 877.

Cardemil, E. V., Reivich, K. J., \& Seligman, M. E. P. (2002). The prevention of depressive symptoms in low-income minority middle school students. Prevention \& Treatment, $5(1), 8 \mathrm{a}$.

Chamberlain, P., \& Reid, J. B. (1998). Comparison of two community alternatives to 
incarceration for chronic juvenile offenders. Journal of consulting and clinical psychology, 66(4), 624-33.

Chorpita, B. F., \& Daleiden, E. L. (2009). Mapping evidence-based treatments for children and adolescents: application of the distillation and matching model to 615 treatments from 322 randomized trials. Journal of consulting and clinical psychology, 77(3), 56679. doi: $10.1037 / \mathrm{a} 0014565$.

Chorpita, B. F., Daleiden, E. L., \& Weisz, J. R. (2005). Modularity in the design and application of therapeutic interventions. Applied and Preventive Psychology, 11, 141156. doi: 10.1016/j.appsy.2005.05.002.

Chorpita, B. F., \& Donkervoet, C. M. (2005). Implementation of the Felix Consent Decree in Hawaii: The impact of policy and practice development efforts on service delivery. In R. G. Steele \&M. C. Roberts (Eds.), Handbook of mental health services for children, adolescents, and families. New York: Kluwer.

Chorpita, B. D., \& Nakamura, B. J. (2004). Four considerations for dissemination of intervention innovations. Clinical Psychology: Science and Practice, 11, 364-367.

Chorpita, B. F., \& Southam-Gerow, M. A. (2006). Treatment of anxiety disorders in youth. In E. J. Mash \& R. A. Barkley (Eds.), Treatment of Childhood Disorders ( ${ }^{\text {rd }}$ ed., pp. 271-335). New York: Guilford Press.

Chorpita, B. F., Yim, L. M., Donkervoet, J. C., Arensdorf, A., Amundsen, M. J., McGee, C., et al. (2002). Toward large-scale implementation of empirically supported treatments for children: A review and observations by the Hawaii Empirical Basis to Services Task Force. Clinical Psychology: Science \& Practice, 9, 165-190.

Chorpita, B. F., Yim, L., Moffitt, C. E., Umemoto, L. A., \& Francis, S. E. (2000). Behaviour Research and Therapy, 38 .

Christophersen, E. R., \& Mortweet, S. L. (2001). Diagnosis and management of anxiety disorders. Treatments that work with children: Empirically supported strategies for managing childhood problems (pp. 49-78). Washington, DC: American Psychological Association. doi: 10.1037/10405-002.

Clarke, G. N., Hornbrook, M., Lynch, F., Polen, M., Gale, J., O’Connor, E., et al. (2002). Journal of the American Academy of Child and Adolescent Psychiatry, 41(3), 305-13. doi: 10.1097/00004583-200203000-00010.

Cohen, J. (1988). Statistical power analysis for the behavioral sciences (2nd ed.). Hillsdale, NJ: Erlbaum.

Costello, E. J., Egger, H. L., Angold, A. (2005). The developmental epidemiology of anxiety disorders: Phenomenology, prevalence, and comorbidity. Child \& 
Adolescent Psychiatric Clinics of North America, 14, 631-648.

Costello, E. J., Erkanli, A., \& Angold, A. (2006). Journal of child psychology and psychiatry, and allied disciplines, 47(12), 1263-71. doi: 10.1111/j.1469-7610.2006.01682.x.

Costello, E. J., Mustillo, S., Erkanli, A., Keeler, G., \& Angold, A. (2003). Prevalence and development of psychiatric disorders in children and adolescents. Archives of General Psychiatry, 60, 837-844.

David-Ferdon, C., \& Kaslow, N. J. (2008). Journal of clinical child and adolescent psychology, 37(1), 62-104. doi: 10.1080/15374410701817865.

de Ross, R., Gullone, E., \& Chorpita, B. F. (2002). The Revised Child Anxiety and Depression Scale: A psychometric investigation with Australian youth. Behaviour Change, 19, 90-101.

Dulcan, M. K. (2005). Practitioner perspectives on evidence-based practice. Child and Adolescent Psychiatric Clinics of North America, 14(2), 225-40, vii. doi: 10.1016/j.chc.2004.04.005.

Ehrenreich-May, J. T., Southam-Gerow, M. A., Hourigan, S. E., Wright, L. R., Pincus, D. B., $\&$ Weisz, J. R. (2010). Differences in characteristics of anxious and depressed youth seen in two different clinical contexts. Administration and Policy in Mental Health and Mental Health Services Research, 1-14.

Evans, S. W. (2005). Introduction to special issue on school-based treatment of children and adolescents with ADHD. Journal of Attention Disorders, 9(1), 245-247. doi: $10.1177 / 108705470500900102$.

Eyberg, S. M., Nelson, M. M, \& Boggs, S. R. (2008). Evidence-based psychosocial treatments for children and adolescents with disruptive behavior. Journal of Clinical Child \& Adolescent Psychology, 37, 215-237.

Fergusson, D. M., Horwood, L. J., \& Lynskey, M. T. (1993). Prevalence and comorbidity of DSM-III-R diagnoses in a birth cohort of 15 year olds. Journal of the American Academy of Child \& Adolescent Psychiatry, 32, 1127-1134.

Flannery-Schroeder, E., Suveg, C., Safford, S., Kendall, P. C., \& Webb, A. (2004). comorbid externalizing disorders and child anxiety treatment outcomes. Behaviour Change, 21, 14-25.

Garcia, A. M., Sapyta, J. J., Moore, P. S., Freeman, J. B., Franklin, M. E., March, J. S., et al. (2010). Predictors and moderators of treatment outcome in the Pediatric Obsessive Compulsive Treatment Study (POTS I). Journal of the American Academy of Child and Adolescent Psychiatry, 49, 1024-33. doi: 10.1016/j.jaac.2010.06.013. 
Gaynor, S. T., Baird, S .C., \& Nelson-Gray, R. O. (1999). Application of time-series (single subject) designs in clinical psychology. In P.C. Kendall, J.N. Butcher, \& G.N. Holmbeck (Eds.), Handbook of research methods in clinical psychology (2nd ed.) (pp. 297-329). Hoboken, NJ: John Wiley \& Sons Inc.

Goodman, R. (2001). Psychometric properties of the Strengths and Difficulties Questionnaire (SDQ). Journal of the American Academy of Child and Adolescent Psychiatry, 40, 1337-1345.

Gottfredson, G., \& Gottfredson, D. (2001). What schools do to prevent problem behavior and promote safe environments. Journal of Educational and Psychological Consultation, 12(4), 313-344. doi: 10.1207/S1532768XJEPC1204_02.

Hammen, C. \& Rudolph, K.D. (2003). Childhood mood disorders. In E. J. Mash \& R.A. Barkley (Eds.), Child psychopathology (Vol. 2, pp. 233-278). New York: Guilford.

Harper, G. W., Bangi, A. K., Contreras, R., Pedraza, A., Tolliver, M., \& Vess, L. (2004). Diverse phases of collaboration: Working together to improve community-based HIV interventions for adolescents. American Journal of Community Psychology, 33, 193 204.

Henggeler, S. W., Schoenwald, S. K., Borduin, C. M., Rowland, M.D.,\& Cunningham, P.B. (1998), Multisystemic treatment of antisocial behavior in children and adolescents. New York and London: Guilford Press.

Henggeler, S. W., Schoenwald, S. K., \& Pickrel, S. G. (1995). Multisystemic therapy: bridging the gap between university- and community-based treatment. Journal of consulting and clinical psychology, 63(5), 709-17.

Hoagwood, K., Burns, B. J., \& Weisz, J. (2002). A profitable conjunction: From science to service in children's mental health. In B. J. Burns \& K. Hoagwood (Eds.), Community based interventions for youth with severe emotional disturbances (pp. 327-338). New York: Oxford University Press.

Hoagwood, K., Jensen, P. S., Petti, T., \& Burns, B. J. (1996). Outcomes of mental health care for children and adolescents: I. A comprehensive conceptual model. Journal of the American Academy of Child and Adolescent Psychiatry, 35, 1055-1063.

Henggeler, S. W., Melton, G. B., Brondino, M. J., Scherer, D.G.,\& Hanley, J. H. (1997). Multisystemic therapy with violent and chronic juvenile offenders and their families: The role of treatment fidelity in successful dissemination. Journal of Consulting and Clinical Psychology, 65, 821-833.

Henggeler, S.W., Melton, G.B., \& Smith, L.A. (1992). Family preservation using multisystemic therapy: An effective alternative to incarcerating serious juvenile offenders. Journal of Consulting and Clinical Psychology, 60, 953-961. 
Hoagwood, K., Burns, B. J., \&Weisz, J. R. (2002).A profitable conjunction: From science to service in children's mental health. In B. J. Burns \& K. Hoagwood (Eds.), Community treatment for youth: Evidence-based interventions for severe emotional And behavioral disorders (pp. 327-338). New York: Oxford University Press.

Hoagwood, K., \& Olin, S. (2002). The NIMH blueprint for change report: Research priorities in child and adolescent mental health. Journal of the American Academy of Child \& Adolescent Psychiatry, 41, 760-767.

Jason, L.A., Keys, C.B., Suarez-Balcazar, Y., Taylor, R.R., \& Davis, M.I. (Eds.). (2004). Participatory community research: Theories and methods in action. Washington, DC: American Psychological Association.

Jensen, P. S., Weersing, R., Hoagwood, K. E., \& Goldman, E. (2005). What Is the Evidence for Evidence-Based Treatments? A Hard Look at Our Soft Underbelly. Mental Health Services Research, 7, 53-74. doi: 10.1007/s11020-005-1965-3.

Kaufman, J., Birmaher, B., Brent, D. A., Rao, U., \& Ryan, N. D. (1997). Kiddie Schedule for Affective Disorders and Schizophrenia - Present and Lifetime Version (K-SADS-PL). Pittsburgh, PA: Western Psychiatric Institute and Clinic.

Kazdin A. E. (1982). Single-case research designs: Methods for clinical and applied settings. New York, NY: Oxford University Press.

Kazdin, A. E. (1995). Child, parent, and family dysfunction as predictors of outcome in cognitive-behavioral treatment of antisocial children. Behaviour Research and Therapy, 33, 271-281.

Kazdin, A. E. (2000). Psychotherapy for children and adolescents: Directions for research and practice. New York: Oxford University Press.

Kazdin, A. E. \& Whitley, M. K. (2006). Comorbidity, case complexity, and effects of evidence-base treatment for children referred for disruptive behavior. Journal of Consulting and Clinical Psychology, 74, 455-467.

Kendall, P. C., Brady, E. U., \& Verduin, T. L. (2001). Comorbidity in childhood anxiety disorders and treatment outcome. Journal of the American Academy of Child and Adolescent Psychiatry, 40, 787-794.

Kendall, P. C., Flannery-Schroeder, E. C., \& Ford, J. D. (1999). Therapy outcome research methods. In P.C. Kendall, J.N. Butcher, \& G.N. Holmbeck, (Eds.), Handbook of research methods in clinical psychology (2nd ed.) (pp. 330-363). Hoboken, NJ: John Wiley \& Sons.

Kendall P. C., \& Grove, W. M. (1988). Normative comparisons in therapy outcome. 
Behavioral Assessment, 10, 147-158.

Kendall, P. C., Kane, M., Howard, B., \& Siqueland, L. (1990). Cognitive-behavioral treatment of anxious children: Treatment manual. Ardmore, PA: Workbook.

Kerfoot, M., Harrington, R., Harrington, V., Rogers, J., \& Verduyn, C. (2004). European Child \& Adolescent Psychiatry, 13(2), 92-9. doi: 10.1007/s00787-004-0362-6.

Koehler, M. J., \& Levin, J. R. (1998). Regulated randomization: A potentially sharper analytical tool for the multiple-baseline design. Psychological Methods, 3, 206-217.

Levant, R. F. (2004). The empirically validated treatments movement: A practitioner/educator perspective. Clinical Psychology: Science and Practice, 11(2), 219-224. doi: 10.1093/clipsy/bph075.

Leve, L. D., Chamberlain, P., \& Reid, J. B. (2005). Intervention outcomes for girls referred from juvenile justice: Effects on delinquency. Journal of consulting and clinical psychology, 73(6), 1181-5. doi: 10.1037/0022-006X.73.6.1181.

Lewinsohn, P. M., \& Essau, C. A. (2002). Depression in adolescents. In I.H. Gotlib \& C.L. Hammen (Eds.), Handbook of depression (pp. 541-559). New York: Guilford Press.

Lewinsohn, P. M., Rohde, P., \& Seeley, J. R. (1998). Clinical psychology review, 18(7), 76594. Retrieved from http://www.ncbi.nlm.nih.gov/pubmed/9827321.

Loeber, R., Burke, J. D., Lahey, B. B., Winters, A., \& Zera, M. (2000). Oppositional defiant and conduct disorder: A review of the past 10 years, part I. Journal of the American Academy of Child \& Adolescent Psychiatry, 39, 1468-1484.

Martin, G. W., Herie, M. A, Turner, B. J., \& Cunningham, J. A. (1998). A social marketing model for disseminating research-based treatments to addictions treatment providers. Addiction, 93(11), 1703-1715. doi: 10.1046/j.1360-0443.1998.931117038.x.

McClendon, D. T., Warren, J. S., Green, K. M., Burlingame, G. M., Eggett, D. L., \& McClendon, R. J. (2011). Sensitivity to change of youth treatment outcome measures: a comparison of the CBCL, BASC-2, and Y-OQ. Journal of Clinical Psychology, 67, 111-25. doi: 10.1002/jclp.20746.

McGee, R., Feehan, M., Williams, S., Partridge, F., Silva, P.A., \& Kelly, S. (1990). DSM-III disorders in a large sample of adolescents. Journal of the American Academy of Child and Adolescent Psychiatry, 29, 611-619.

McMahon, R. J., \& Frick, P. J. (2007). Conduct and oppositional disorders. In E.J. Mash \& R.A. Barkley (Eds.), Assessment of childhood disorders (4th Ed.) (pp. 132-183). New York: Guilford Press. 
Middlestadt, S. E., Schechter, C., Peyton, J., \& Tjugum, B. (1997). Community involvement in health planning: Lessons learned from practicing social marketing in a context of community control, participation, and ownership. In M. E. Goldberg, M. Fishbein, \& S. E. Middlestadt (Eds.), Social marketing: Theoretical and practical perspectives (pp. 291-311). Mahwah, NJ: Lawrence Erlbaum Associates.

Miller, L. M., Southam-Gerow, M. a, \& Allin, R. B. (2008). Who Stays in Treatment? Child and Family Predictors of Youth Client Retention in a Public Mental Health Agency. Child Youth Care Forum, 37, 153-170. doi: 10.1007/s10566-008-9058-2.

Murphy, M., Cowan, R., \& Sederer, L. (2001). Disorders of childhood and adolescence. Blueprints in Psychiatry, Second Edition. Malden, Mass: Blackwell Science, Inc., 4041.

Nathan, P. E., Stuart, S. P., \& Dolan, S. L. (2000). Research on psychotherapy efficacy and effectiveness: Between Scylla and Charybdis? Psychological Bulletin, 126, 964-981.

Norquist, G., Lebowitz, B., \& Hyman, S. (1999). Expanding the frontier of treatment research. Prevention \& Treatment, 2(1). doi: 10.1037//1522-3736.2.1.21a.

Pelham, W.E., \& Fabiano, G.A. (2008). Evidence-based psychosocial treatments for attention-deficit/hyperactivity disorder. Journal of Clinical Child \& Adolescent Psychology, 37, 184-214.

Photos, V.I., Michel, B.D., \& Nock, M. K. (2008). Single case research. In M. Hersen \& A.M. Gross (Eds.), Handbook of clinical psychology, vol 1: Adults (pp. 224-245). Hoboken, NJ: John Wiley \& Sons.

Rohde, P., Clarke, G.N., Mace, D.E., Jorgensen, J.S., \& Seeley, J.R. (2004). An efficacy/ effectiveness study of cognitive-behavioral treatment for adolescents with comorbid major depression and conduct disorder. Journal of the American Academy of Child \& Adolescent Psychiatry, 43, 660-668.

Rohde, P., Seeley, J. R., Kaufman, N. K., Clarke, G. N., \& Stice, E. (2006). Predicting time to recovery among depressed adolescents treated in two psychosocial group interventions. Journal of Consulting and Clinical Psychology, 74, 80-8. doi: 10.1037/0022-006X.74.1.80.

Rudolph, K.D., Hammen, C., \& Daley, S. E. (2006). Mood Disorders. In D.A. Wolfe \& E.J. Mash (Eds.), Behavioral and emotional disorders in adolescents: Nature, assessment, and treatment (pp. 300-342). New York: Guilford Publications. 
Salzer, M. S., Bickman, L., \& Lambert, E. W. (1999). Dose-effect relationship in children's psychotherapy services. Journal of Consulting and Clinical Psychology, 67, 228-238. doi: 10.1037/0022-006X.67.2.228.

Schoenwald, S. K. (2010). From policy pinball to purposeful partnership: The policy contexts of multisystemic therapy transport and dissemination. In J. R. Weisz \& A. E. Kazdin (Eds.), Evidence-based psychotherapies for children and adolescents $\left(2^{\text {nd }}\right.$ ed.) (pp. 538-553). New York: Guilford Press.

Schoenwald, S.K., \& Hoagwood, K. (2001). Effectiveness and dissemination research: Their mutual roles in improving mental health services for children and adolescents. Emotional \& Behavioral Disorders in Youth, 2, 3-4; 18-20.

Shaffer, D., Gould, M.S., Brasic, J., Ambrosini, R., Fisher, P., Bird, H., et al. (1983). A children's global assessment scale (CGAS). Archives of General Psychiatry, 40, 1228-1231.

Shirk, S. R., Kaplinski, H., \& Gudmundsen, G. (2008). School based cognitive-behavioral therapy for adolescent depression: A benchmarking study. Journal of Emotional and Behavioral Disorders, 17(2), 106-117. doi: 10.1177/1063426608326202.

Sidman, M. (1960). Tactics of scientific research. Oxford, England: Basic Books.

Silverman, W.K., Pina, A.A., Viswesvaran, C. (2008). Evidence-based psychosocial treatments for phobic and anxiety disorders in children and adolescents. Journal of Clinical Child \& Adolescent Psychology, 37, 105-130.

Smith-Boydston, J. M. \& Nelson, T. D. (2008). Adoption of evidence-based treatments in community settings: Obstacles and opportunities. In R.G. Steele, T.D. Elkin, \& M.C. Roberts (Eds.), Handbook of evidence-based therapies for children and adolescents: Bridging science and practice (pp. 521-535). New York: Springer. doi: 10.1007/978 0-387-73691-4_29

Southam-Gerow, M. A. (2004). Some reasons that mental health treatments are not technologies: Toward treatment development and adaptation outside labs. Clinical Psychology: Science \& Practice, 11, 186-189.

Southam-Gerow, M. A. (2005, Summer). Using partnerships to adapt evidence-based mental health treatments for use outside labs. Report on Emotional \& Behavioral Disorders in Youth, 5, 58-60, 77-79.

Southam-Gerow, M. A., Hourigan, S. E., \& Allin, R. B. (2009). Adapting evidence-based mental health treatments in community settings: preliminary results from a partnership approach. Behavior Modification, 33(1), 82-103.

Southam-Gerow, M. A., Marder, A. M., Austin, A. (2008). Dissemination of evidence-based treatments for children and families in practice settings. In R.G. Steele, T.D. Elkin, \& 
M.C. Roberts (Eds.), Handbook of evidence-based therapies for children and adolescents: Bridging science and practice, Issues in clinical child psychology (pp. 447-469). New York: Springer. doi: 10.1007/978-0-387-73691-4_25

Southam-Gerow, M. A., \& Chorpita, B. F. (2007). Anxiety in children and adolescents. In E.J. Mash \& R.A. Barkley (Eds.), Assessment of childhood disorders (4th Ed.) (pp. 347-397). New York: Guilford Press.

Southam-Gerow, M. A., Chorpita, B. F., Miller, L. M., \& Gleacher, A. A. (2008). Are children with anxiety disorders privately referred to a university clinic like those referred from the public mental health system. Administration and Policy in Mental Health and Mental Health Services Research, 35, 168-180.

Southam-Gerow, M. A., Kendall, P. C., \& Weersing, V. R. (2001). Examining outcome variability: correlates of treatment response in a child and adolescent anxiety clinic. Journal of Clinical Child Psychology, 30, 422-436.

Southam-Gerow, M. A., Silverman, W. K., \& Kendall, P. C. (2006). Client similarities and differences in two childhood anxiety disorders research clinics. Journal of Clinical Child and Adolescent Psychology, 35(4), 528-38. doi: 10.1207/s15374424jccp3504_4.

Southam-Gerow, M. A., Weisz, J. R., Chu, B. C., McLeod, B. D., Gordis, E. B., \& ConnorSmith, J. K. (2010). Does cognitive behavioral therapy for youth anxiety outperform usual care in community clinics? An initial effectiveness test. Journal of the American Academy of Child and Adolescent Psychiatry, 49(10), 1043-52. doi: 10.1016/j.jaac.2010.06.009.

Southam-Gerow, M. A., Weisz, J. R., \& Kendall, P. C. (2003). Childhood anxiety disorders in research and service clinics: Preliminary examination of differences and similarities. Journal of Clinical Child and Adolescent Psychology, 32, 375-385.

Street, L. L., Niederehe, G., \& Lebowitz, B. (2000). Toward greater public health relevance for psychotherapeutic interventions research: An NIMH workshop report. Clinical Psychology: Science and Practice , 7, 127-137.

Sullivan, M. J., \& Kelly, J. B. (2001). Legal and psychological management of cases with an alienated child. Family Court Review, 39, 299-315.

Taylor, R. R., Jason, L. A., Keys, C.B., Suarez-Balcazar, Y., Davis, M. I., Durlak, J. A., et al. (2004). Capturing theory and methodology in participatory research. In L. A. Jason, C. B. Keys, Y. Suarez-Balcazar, R. R. Taylor \& M. I. Davis (Eds.), Participatory community research: Theories and methods in action (pp. 3-14).Washington, DC: American Psychological Association.

U.S. Department of Health and Human Services (1999). Mental health: A report of the Surgeon General - Executive summary. Rockville, MD: U.S. Department of Health 
and Human Services, Substance Abuse and Mental Health Services Administration, Center for Mental Health Services, National Institutes of Health, National Institute of Mental Health.

U.S. Public Health Service (2000). Report of the surgeon general's conference on children's mental health: A national action agenda. Washington, DC: Department of Health and Human Services.

Verduin, T. L., \& Kendall, P. C. (2003). Differential occurrence of comorbidity within childhood anxiety disorders. Journal of Clinical Child \& Adolescent Psychology, 32, 290-295.

Walker, H. M., Ramsey, E., \& Gresham, F. M. (2003). Antisocial behavior in school: Evidence-based practices (2nd ed.). Belmont, CA: Wadsworth/Thomson Learning.

Weersing, V. R., Iyengar, S., Kolko, D. J., Birmaher, B., \& Brent, D. a. (2006). Effectiveness of cognitive-behavioral therapy for adolescent depression: A benchmarking study. Behavior therapy, 37(1), 36-48. doi: 10.1016/j.beth.2005.03.003.

Weersing, V. R., \& Weisz, J. R. (2002). Journal of Consulting and Clinical Psychology, 70(2), 299 -310. doi: 10.1037//0022-006X.70.2.299.

Weiss, B., Catron, T., \& Harris, V (2000). A 2-year follow-up of the effectiveness of traditional child psychotherapy. Journal of Counseling and Clinical Psychology, 68, 1094-1101.

Weiss, B., Harris, V., Catron, T., \& Han, S. S. (2003). Efficacy of the RECAP intervention program for children with concurrent internalizing and externalizing problems. Journal of Consulting and Clinical Psychology, 71(2), 364-374. doi: 10.1037/0022006X.71.2.364.

Westen, D., Novotny, C. M., \& Thompson-Brenner, H. (2004). The empirical status of empirically supported psychotherapies: Assumptions, findings, and reporting in controlled clinical trials. Psychological Bulletin, 130, 631-663.

Weisz, J. R. (2000). Lab-clinic differences and what we can do about them: The clinic-based treatment development model. Clinical Child Psychology Newsletter, 15, 1-2.

Weisz, J.R., Hawley, K.M., Pilkonis, P.A., Woody, S.R., \& Follette, W.C. (2000). Stressing the (other) three Rs in the search for empirically supported treatments: Review procedures, research quality, and relevance to clinical practice. Clinical Psychology: Science and Practice, 7, 243-258.

Weisz, J.R., Jensen-Doss, A., Hawley, K.M. (2006). Evidence-based youth psychotherapies versus usual clinical care: A meta-analysis of direct comparisons. American Psychologist, 61, 671-689. 
Weisz, J.R., \& Kazdin, A.E. (2003). Concluding thoughts: Present and future of evidence based psychotherapies for children and adolescents. In A.E. Kazdin \& J.R. Weisz (Eds.), Evidence-based psychotherapies for children and adolescents (pp. 439-451). New York: Guilford.

Weisz, J.R., Moore, P.S., Southam-Gerow, M.A., Weersing, B.R., Valeri, S.M., \&McCarty, C.A. (1999). Therapist's manual: Primary and secondary control Enhancement training program. University of California, Los Angeles.

Weisz, J.R., Southam-Gerow, M.A., Gordis, E.B., \& Connor-Smith, J. (2003). Primary and secondary control enhancement training for youth depression. In A.E. Kazdin \& J.R. Weisz (Eds.), Evidence-Based Psychotherapies for Children and Adolescents (pp. 165- 183). New York: Guilford Press.

Weisz, J. R., Southam-Gerow, M. A, Gordis, E. B., Connor-Smith, J. K., Chu, B. C., Langer, D. A, ... Weiss, B. (2009). Cognitive-behavioral therapy versus usual clinical care for youth depression: An initial test of transportability to community clinics and clinicians. Journal of Consulting and Clinical Psychology, 77(3), 383-396. doi: 10.1037/a0013877.

Weisz, J. R., Weiss, B., Han, S. S., Granger, D. A., \& Morton, T. (1995). Effects of psychotherapy with children and adolescents revisited: A meta-analysis of treatment outcome studies. Psychological Bulletin, 117, 450-468. 
Vita

Alexis Michelle Quinoy was born on July 13, 1983 in Miami, Florida and is an American citizen. She graduated from Chattahoochee High School, Alpharetta, Georgia in 2001. She received her Bachelor of Science in Psychology with a minor in Spanish for Native Speakers and graduated Cum Laude from The University of Florida, Gainesville, Florida in 2005. She is a member of Phi Beta Kappa. Ms. Quinoy is currently enrolled in the Clinical Psychology Ph.D. program at Virginia Commonwealth University. 